Supporting information:

\title{
Computational studies of metal-ligand bond enthalpies across the transition metal series
}

\author{
Jamal Uddin, Christine M. Morales, James H. Maynard, and Clark R. Landis* \\ Department of Chemistry, University of Wisconsin-Madison, 1101 University Avenue, \\ Madison, WI 53706
}

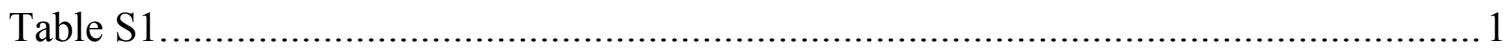

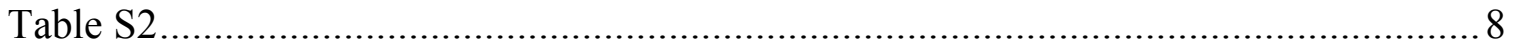

Selected Cartesian Coordinates and Total Energies .................................................. 11

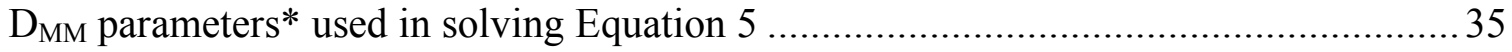

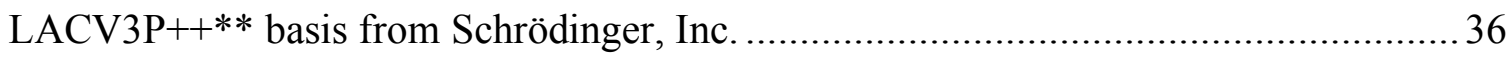

Table S1. Computational data for neutral, valency-saturated compounds $\mathrm{H}_{\mathrm{n}} \mathrm{M}-\mathrm{L}$ for different ligands L, at B3LYP and CCSD(T) levels of theory using LACV3P $++* *$ basis set: Hybridization at the metal center $(\% \mathrm{~s})$, bond enthalpy (DE, $\mathrm{kcal} / \mathrm{mol})$, polarization toward the metal center (Pol, \%M), charge on the ligand $(\mathrm{q}(\mathrm{L}))$, bond length (rM-L, $\AA$ ), and charge on the metal $(\mathrm{q}(\mathrm{M}))$. Hybridization, polarization, and charges were determined from Natural Bond Orbital analyses of the final wavefunction.

\begin{tabular}{ccccccccc} 
Ligand & Method & Metal & \%s & DE (kcal) & Pol (\%M) & q(L) & rM-L & q(M) \\
\hline $\mathrm{H}$ & B3LYP & $\mathrm{Sc}$ & 27.2 & 68.99 & 23.61 & -0.52 & 1.804 & 1.58 \\
& $\mathrm{Ti}$ & 25.23 & 58.51 & 34.7 & -0.31 & 1.688 & 1.23 \\
& $\mathrm{~V}$ & 20.13 & 42.83 & 43.41 & -0.13 & 1.6068 & 0.64 \\
& $\mathrm{Cr}$ & 16.75 & 56.5 & 53.31 & 0.07 & 1.504 & -0.28 \\
& $\mathrm{Mn}$ & 19.9 & 55.9 & 51.07 & 0.01 & 1.498 & -0.05 \\
& $\mathrm{Fe}$ & 25 & 43.6 & 47.54 & -0.05 & 1.472 & 0.21 \\
& $\mathrm{Co}$ & 33.15 & 47.8 & 46.74 & -0.06 & 1.433 & 0.19 \\
& $\mathrm{Ni}$ & 48.19 & 51.5 & 44.62 & -0.11 & 1.417 & 0.21 \\
& $\mathrm{Cu}$ & 93.2 & 61.91 & 33.64 & -0.33 & 1.473 & 0.33 \\
& $\mathrm{Y}$ & 28.87 & 70.56 & 21.2 & -0.57 & 1.977 & 1.72 \\
& $\mathrm{Zr}$ & 25 & 74.38 & 30.47 & -0.39 & 1.85 & 1.57 \\
& $\mathrm{Nb}$ & 20.02 & 58.93 & 39.88 & -0.2 & 1.7532 & 1.01 \\
& $\mathrm{Mo}$ & 16.71 & 65.54 & 49.97 & -0.01 & 1.667 & 0.07 \\
& $\mathrm{Tc}$ & 19.85 & 67.48 & 53.81 & 0.06 & 1.603 & -0.31 \\
& $\mathrm{Ru}$ & 24.95 & 70.8 & 53.99 & 0.07 & 1.565 & -0.29
\end{tabular}




\begin{tabular}{|c|c|c|c|c|c|c|c|c|}
\hline & & $\mathrm{Rh}$ & 33.14 & 70.1 & 52.89 & 0.05 & 1.522 & -0.14 \\
\hline & & $\mathrm{Pd}$ & 48.15 & 64.3 & 45.09 & -0.02 & 1.522 & 0.05 \\
\hline & & $\mathrm{Ag}$ & 94.76 & 52.12 & 33.99 & -0.33 & 1.629 & 0.33 \\
\hline & & $\mathrm{La}$ & a & 66.98 & $a$ & $a$ & 2.128 & $a$ \\
\hline & & $\mathrm{Hf}$ & 25 & 81.59 & 27.56 & -0.44 & 1.833 & 1.79 \\
\hline & & $\mathrm{Ta}$ & 20.02 & 66.6 & 36.4 & -0.27 & 1.7552 & 1.34 \\
\hline & & W & 16.73 & 67.78 & 46.06 & -0.1 & 1.685 & 0.48 \\
\hline & & $\mathrm{Re}$ & 19.2 & 62.22 & 51.23 & 0.02 & 1.624 & -0.09 \\
\hline & & Os & 25 & 77.9 & 48.56 & -0.03 & 1.601 & 0.13 \\
\hline & & Ir & 33.12 & 80.29 & 52.68 & -0.05 & 1.547 & -0.14 \\
\hline & & $\mathrm{Pt}$ & 54.22 & 80.92 & 54.22 & 0.07 & 1.52 & -0.14 \\
\hline & & $\mathrm{Au}$ & 82.96 & 70.97 & 48 & -0.05 & 1.543 & 0.05 \\
\hline & $\operatorname{ccsD}(T)$ & Sc & 27.2 & 67.76099 & 23.61 & -0.52 & 1.804 & 1.58 \\
\hline & & $\mathrm{Ti}$ & 25.23 & 57.66762 & 34.7 & -0.31 & 1.688 & 1.23 \\
\hline & & V & 20.13 & 42.83 & 43.41 & -0.13 & 1.6068 & 0.64 \\
\hline & & $\mathrm{Cr}$ & 16.75 & 61.31835 & 53.31 & 0.07 & 1.504 & -0.28 \\
\hline & & $\mathrm{Mn}$ & 19.9 & 55.9 & 51.07 & 0.01 & 1.498 & -0.05 \\
\hline & & $\mathrm{Fe}$ & 25 & 47.03843 & 47.54 & -0.05 & 1.472 & 0.21 \\
\hline & & Co & 33.15 & 47.8 & 46.74 & -0.06 & 1.433 & 0.19 \\
\hline & & $\mathrm{Ni}$ & 48.19 & 53.80084 & 44.62 & -0.11 & 1.417 & 0.21 \\
\hline & & $\mathrm{Cu}$ & 5.77 & 61.91 & 33.64 & -0.33 & 1.473 & 0.33 \\
\hline & & $Y$ & 28.87 & 75.4439 & 21.2 & -0.57 & 1.977 & 1.72 \\
\hline & & $\mathrm{Zr}$ & 25 & 73.33302 & 30.47 & -0.39 & 1.85 & 1.57 \\
\hline & & $\mathrm{Nb}$ & 20.02 & 58.93 & 39.88 & -0.2 & 1.7532 & 1.01 \\
\hline & & Mo & 16.71 & 64.02555 & 49.97 & -0.01 & 1.667 & 0.07 \\
\hline & & Tc & 19.85 & 67.48 & 53.81 & 0.06 & 1.603 & -0.31 \\
\hline & & $\mathrm{Ru}$ & 24.95 & 71.85015 & 53.99 & 0.07 & 1.565 & -0.29 \\
\hline & & $\mathrm{Rh}$ & 33.14 & 70.1 & 52.89 & 0.05 & 1.522 & -0.14 \\
\hline & & $\mathrm{Pd}$ & 48.15 & 62.97045 & 45.09 & -0.02 & 1.522 & 0.05 \\
\hline & & $\mathrm{Ag}$ & 4.24 & 52.12 & 33.99 & -0.33 & 1.629 & 0.33 \\
\hline & & La & $a$ & 66.98 & $a$ & $a$ & 2.128 & $a$ \\
\hline & & $\mathrm{Hf}$ & 25 & 73.90041 & 27.56 & -0.44 & 1.833 & 1.79 \\
\hline & & Ta & 20.02 & 66.6 & 36.4 & -0.27 & 1.7552 & 1.34 \\
\hline & & W & 16.73 & 66.15437 & 46.06 & -0.1 & 1.685 & 0.48 \\
\hline & & $\operatorname{Re}$ & 19.2 & 62.22 & 51.23 & 0.02 & 1.624 & -0.09 \\
\hline & & Os & 25 & 78.66528 & 48.56 & -0.03 & 1.601 & 0.13 \\
\hline & & Ir & 33.12 & 80.29 & 52.68 & -0.05 & 1.547 & -0.14 \\
\hline & & $\mathrm{Pt}$ & 54.22 & 83.8113 & 54.22 & 0.07 & 1.52 & -0.14 \\
\hline & & $\mathrm{Au}$ & 87.24 & 70.5 & 37.31 & -0.26 & 1.543 & 0.26 \\
\hline $\mathrm{CH} 3$ & B3LYP & Sc & 21.11 & 65.43 & 18.07 & -0.61 & 2.162 & 1.67 \\
\hline & & $\mathrm{Ti}$ & 19.34 & 59.19 & 28.3 & -0.37 & 2.029 & 1.27 \\
\hline & & $\mathrm{V}$ & 9.28 & 44.96 & 34.59 & -0.22 & 1.974 & 0.62 \\
\hline & & $\mathrm{Cr}$ & 18.94 & 52.28 & 40.03 & -0.08 & 1.917 & -0.16 \\
\hline & & $\mathrm{Mn}$ & 18.77 & 45.09 & 37.86 & -0.06 & 1.899 & 0.34 \\
\hline & & $\mathrm{Fe}$ & 20.69 & 43.26 & 41.14 & -0.15 & 1.846 & 0.33 \\
\hline & & Co & 30.09 & 41.01 & 39.2 & -0.22 & 1.858 & 0.36 \\
\hline & & $\mathrm{Ni}$ & 42.87 & 44.08 & 38.77 & -0.23 & 1.841 & 0.35 \\
\hline & & $\mathrm{Cu}$ & 91.36 & 52.09 & 31.35 & -0.38 & 1.931 & 0.38 \\
\hline & & $Y$ & 24.85 & 63.92 & 24.85 & -0.66 & 2.344 & 1.8 \\
\hline & & $\mathrm{Zr}$ & 20.35 & 72.33 & 26.1 & -0.43 & 2.201 & 1.63 \\
\hline
\end{tabular}




\begin{tabular}{|c|c|c|c|c|c|c|c|c|}
\hline & & $\mathrm{Nb}$ & 17.16 & 57.18 & 29.99 & -0.22 & 2.135 & 1.02 \\
\hline & & Mo & 13.35 & 63.82 & 36.4 & 0 & 2.074 & 0.17 \\
\hline & & Tc & 12.86 & 54.98 & 40.41 & -0.14 & 2.022 & 0.06 \\
\hline & & $\mathrm{Ru}$ & 19.24 & 58.08 & 44.27 & -0.1 & 1.993 & 0.07 \\
\hline & & $\mathrm{Rh}$ & 30.42 & 54.78 & 44.61 & -0.14 & 2.007 & 0.06 \\
\hline & & $\mathrm{Pd}$ & 44.65 & 49.38 & 43.08 & -0.14 & 2.012 & 0.17 \\
\hline & & $\mathrm{Ag}$ & 93.54 & 39.62 & 33.82 & -0.34 & 2.127 & 0.34 \\
\hline & & La & a & 59.29 & $a$ & $a$ & 2.51 & $a$ \\
\hline & & $\mathrm{Hf}$ & 21.31 & 79.62 & 21.34 & -0.52 & 2.181 & 1.87 \\
\hline & & $\mathrm{Ta}$ & 17.87 & 65.41 & 25.54 & -0.44 & 2.132 & 1.44 \\
\hline & & W & 16.58 & 69.59 & 47.36 & -0.06 & 2.07 & 0.56 \\
\hline & & $\mathrm{Re}$ & 18.09 & 61.43 & 51.52 & -0.2 & 2.016 & 0.28 \\
\hline & & Os & 19.7 & 72.12 & 40.64 & -0.17 & 1.995 & 0.29 \\
\hline & & Ir & 30.3 & 66.31 & 43.36 & -0.17 & 2.004 & 0.09 \\
\hline & & $\mathrm{Pt}$ & 42.26 & 72.05 & 46.49 & 0.1 & 2.001 & 0.05 \\
\hline & & $\mathrm{Au}$ & 79.3 & 57.78 & 44.13 & -0.14 & 2.043 & 0.14 \\
\hline $\mathrm{C} 2 \mathrm{H} 1$ & B3LYP & Sc & 23.28 & 122.32 & 12.76 & -0.68 & 2.151 & 1.74 \\
\hline & & $\mathrm{Ti}$ & 22.95 & 108.45 & 20.78 & -0.44 & 2.026 & 1.28 \\
\hline & & $\mathrm{V}$ & 19.11 & 92.84 & 23.89 & -0.42 & 1.991 & 0.68 \\
\hline & & $\mathrm{Cr}$ & 20.07 & 95.29 & 29.63 & -0.32 & 1.921 & 0.16 \\
\hline & & $\mathrm{Mn}$ & 24.33 & 87.33 & 30.21 & -0.32 & 1.855 & 0.39 \\
\hline & & $\mathrm{Fe}$ & 24.26 & 85.07 & 32.06 & -0.30 & 1.807 & 0.33 \\
\hline & & Co & 30.7 & 87.48 & 31.92 & -0.38 & 1.786 & 0.39 \\
\hline & & $\mathrm{Ni}$ & 46.48 & 88.5 & 29.78 & -0.43 & 1.769 & 0.47 \\
\hline & & $\mathrm{Cu}$ & 94.29 & 98.73 & 18.87 & -0.65 & 1.833 & 0.65 \\
\hline & & $Y$ & 24.15 & 122.22 & 12.62 & -0.73 & 2.324 & 1.89 \\
\hline & & $\mathrm{Zr}$ & 22.78 & 124.46 & 18.91 & -0.55 & 2.191 & 1.28 \\
\hline & & $\mathrm{Nb}$ & 18.13 & 106.99 & 23.59 & -0.47 & 2.124 & 1.11 \\
\hline & & Mo & 17.48 & 106.99 & 31.42 & -0.33 & 2.124 & 0.15 \\
\hline & & Tc & 18.4 & 99.75 & 34.59 & -0.23 & 1.949 & 0.08 \\
\hline & & $\mathrm{Ru}$ & 24.04 & 100.55 & 37.48 & -0.25 & 1.911 & 0.11 \\
\hline & & $\mathrm{Rh}$ & 31.13 & 101.48 & 38.58 & -0.31 & 1.889 & 0.13 \\
\hline & & $\mathrm{Pd}$ & 47.03 & 91.11 & 34.1 & -0.37 & 1.905 & 0.31 \\
\hline & & $\mathrm{Ag}$ & 96.51 & 82.9 & 16.35 & -0.65 & 2.045 & 0.65 \\
\hline & & La & $a$ & 117.12 & $a$ & $a$ & 2.505 & $a$ \\
\hline & & $\mathrm{Hf}$ & 22.41 & 133.2 & 17.18 & -0.57 & 2.168 & 1.86 \\
\hline & & $\mathrm{Ta}$ & 20.56 & 117.2 & 21.61 & -0.48 & 2.107 & 1.4 \\
\hline & & W & 20.3 & 117.2 & 27.31 & -0.31 & 2.107 & 0.53 \\
\hline & & $\mathrm{Re}$ & 21.63 & 110.07 & 32.95 & -0.30 & 1.939 & 0.29 \\
\hline & & Os & 23.26 & 119.87 & 36.12 & -0.30 & 1.913 & 0.35 \\
\hline & & Ir & 31.14 & 115.58 & 39.12 & -0.31 & 1.886 & 0.17 \\
\hline & & $\mathrm{Pt}$ & 44.34 & 108.21 & 30.89 & -0.30 & 1.877 & 0.20 \\
\hline & & $\mathrm{Au}$ & 87.76 & 93.83 & 32.94 & -0.41 & 1.931 & 0.41 \\
\hline $\mathrm{C} 2 \mathrm{H} 3$ & B3LYP & Sc & 19.24 & 73.1 & 16.77 & -0.62 & 2.149 & 1.68 \\
\hline & & $\mathrm{Ti}$ & 18.39 & 66.52 & 27 & -0.29 & 2.025 & 1.23 \\
\hline & & $\mathrm{V}$ & 12.75 & 53.17 & 32.78 & -0.22 & 1.964 & 0.54 \\
\hline & & $\mathrm{Cr}$ & 13.46 & 56.99 & 37.84 & -0.08 & 1.96 & -0.12 \\
\hline & & $\mathrm{Mn}$ & 14.6 & 47.06 & 40.38 & -0.08 & 1.858 & 0.08 \\
\hline & & $\mathrm{Fe}$ & 20.19 & 50.94 & 40.12 & -0.16 & 1.827 & 0.31 \\
\hline & & Co & 27.46 & 51.34 & 39.53 & -0.21 & 1.807 & 0.33 \\
\hline
\end{tabular}




\begin{tabular}{|c|c|c|c|c|c|c|c|c|}
\hline & & $\mathrm{Ni}$ & 41.9 & 51.52 & 38.85 & -0.35 & 1.795 & 0.37 \\
\hline & & $\mathrm{Cu}$ & 91.75 & 57.64 & 29.36 & -0.42 & 1.899 & 0.42 \\
\hline & & $Y$ & 18.83 & 72.74 & 15.51 & -0.66 & 2.32 & 1.78 \\
\hline & & $\mathrm{Zr}$ & 20.22 & 78.06 & 23.5 & -0.46 & 2.198 & 1.63 \\
\hline & & $\mathrm{Nb}$ & 14.03 & 68.62 & 31.25 & -0.25 & 2.097 & 0.87 \\
\hline & & Mo & 13.24 & 71.15 & 36.31 & 0 & 2.06 & 0.1 \\
\hline & & Tc & 10.87 & 66.34 & 43.15 & 0.04 & 1.949 & -0.04 \\
\hline & & $\mathrm{Ru}$ & 18.79 & 67.14 & 44.99 & -0.09 & 1.947 & 0.06 \\
\hline & & $\mathrm{Rh}$ & 28.63 & 66.62 & 45.34 & -0.13 & 1.941 & 0.07 \\
\hline & & $\mathrm{Pd}$ & 43.13 & 57.69 & 43.54 & -0.15 & 1.957 & 0.19 \\
\hline & & $\mathrm{Ag}$ & 93.74 & 44.07 & 31.2 & -0.39 & 2.099 & 0.39 \\
\hline & & La & $a$ & 68.75 & $a$ & $a$ & 2.494 & $a$ \\
\hline & & $\mathrm{Hf}$ & 18.37 & 88.84 & 20.75 & -0.46 & 2.156 & 1.75 \\
\hline & & $\mathrm{Ta}$ & 18.19 & 73.73 & 25.68 & -0.47 & 2.118 & 1.35 \\
\hline & & W & 16.51 & 76.44 & 32.61 & -0.21 & 2.053 & 0.46 \\
\hline & & $\mathrm{Re}$ & 12.73 & 74.83 & 39.93 & -0.18 & 1.944 & 0.18 \\
\hline & & Os & 20.17 & 83.76 & 41.56 & -0.2 & 1.953 & 0.29 \\
\hline & & Ir & 29.11 & 79.64 & 44.46 & -0.17 & 1.941 & 0.11 \\
\hline & & $\mathrm{Pt}$ & 40.13 & 76.76 & 46.8 & -0.12 & 1.945 & 0.08 \\
\hline & & $\mathrm{Au}$ & 78.7 & 63.62 & 43.33 & -0.18 & 2.002 & 0.18 \\
\hline $\mathrm{C} 2 \mathrm{H} 5$ & B3LYP & Sc & 19.37 & 58.3 & 19.03 & -0.58 & 2.166 & 1.66 \\
\hline & & $\mathrm{Ti}$ & 17.63 & 52.91 & 29.48 & -0.34 & 2.028 & 1.27 \\
\hline & & $\mathrm{V}$ & 7.89 & 41.06 & 37.09 & -0.15 & 1.941 & 0.55 \\
\hline & & $\mathrm{Cr}$ & 8.47 & 48.23 & 42.42 & -0.02 & 1.916 & -0.18 \\
\hline & & $\mathrm{Mn}$ & 28.86 & 41.66 & 34.6 & -0.2 & 1.898 & 0.32 \\
\hline & & $\mathrm{Fe}$ & 19 & 39.98 & 43.13 & -0.14 & 1.851 & 0.32 \\
\hline & & Co & 27.04 & 37.64 & 40.98 & -0.18 & 1.845 & 0.34 \\
\hline & & $\mathrm{Ni}$ & 39.23 & 39.9 & 41.14 & -0.17 & 1.847 & 0.33 \\
\hline & & $\mathrm{Cu}$ & 90.73 & 44.89 & 33.08 & -0.34 & 1.943 & 0.34 \\
\hline & & $Y$ & 24.22 & 56.01 & 17.01 & -0.64 & 2.348 & 1.78 \\
\hline & & $\mathrm{Zr}$ & 18.8 & 65.14 & 25.15 & -0.45 & 2.204 & 1.63 \\
\hline & & $\mathrm{Nb}$ & 21.49 & 49.89 & 40.71 & -0.37 & 2.152 & 1.09 \\
\hline & & Mo & 17.44 & 54.32 & 53.98 & -0.16 & 2.079 & 0.11 \\
\hline & & Tc & 11.37 & 51.09 & 42.51 & -0.02 & 2.019 & 0.02 \\
\hline & & $\mathrm{Ru}$ & 16.98 & 54.39 & 46.24 & -0.05 & 1.999 & 0.05 \\
\hline & & $\mathrm{Rh}$ & 28.86 & 50.53 & 46.28 & -0.08 & 2.008 & 0.04 \\
\hline & & $\mathrm{Pd}$ & 42.75 & 45.76 & 46.25 & -0.09 & 2.024 & 0.15 \\
\hline & & $\mathrm{Ag}$ & 93.03 & 32.31 & 35.5 & -0.3 & 2.135 & 0.3 \\
\hline & & $\mathrm{La}$ & $a$ & 51.58 & a & $a$ & 2.516 & $a$ \\
\hline & & $\mathrm{Hf}$ & 19.9 & 73.09 & 22.28 & -0.52 & 2.177 & 1.84 \\
\hline & & $\mathrm{Ta}$ & 16.13 & 58.5 & 26.46 & -0.43 & 2.135 & 1.43 \\
\hline & & W & 14.98 & 64.05 & 50.3 & -0.19 & 2.079 & 0.54 \\
\hline & & $\operatorname{Re}$ & 10.04 & 57.16 & 40.03 & -0.1 & 2.039 & 0.1 \\
\hline & & Os & 18.33 & 67.8 & 42.19 & -0.18 & 2.002 & 0.27 \\
\hline & & Ir & 28.7 & 62.33 & 45.03 & -0.1 & 2.013 & 0.06 \\
\hline & & $\mathrm{Pt}$ & 39.84 & 63.07 & 48.49 & -0.06 & 2.016 & 0.02 \\
\hline & & $\mathrm{Au}$ & 77.65 & 52.07 & 45.89 & -0.11 & 2.051 & 0.11 \\
\hline $\mathrm{C} 3 \mathrm{H} 7$ & B3LYP & Sc & 16.41 & 53.12 & 19.6 & -0.58 & 2.166 & 1.66 \\
\hline & & $\mathrm{Ti}$ & 15.81 & 49.27 & 31.14 & -0.63 & 2.037 & 1.25 \\
\hline & & V & 6.57 & 36.1 & 37.39 & -0.29 & 1.986 & 0.61 \\
\hline
\end{tabular}




\begin{tabular}{|c|c|c|c|c|c|c|c|c|}
\hline & & $\mathrm{Cr}$ & 5.62 & 45.84 & 44.69 & -0.03 & 1.915 & -0.22 \\
\hline & & $\mathrm{Mn}$ & 14.61 & 34.95 & 41.92 & -0.1 & 1.946 & 0.3 \\
\hline & & $\mathrm{Fe}$ & 16.95 & 37.46 & 44.89 & -0.1 & 1.856 & 0.31 \\
\hline & & Co & 25.26 & 32.25 & 42.09 & -0.16 & 1.876 & 0.32 \\
\hline & & $\mathrm{Ni}$ & 36.99 & 37.01 & 62.96 & -0.13 & 1.84 & 0.31 \\
\hline & & $\mathrm{Cu}$ & 90.32 & 40.09 & 34.51 & -0.32 & 1.958 & 0.32 \\
\hline & & $\mathrm{Y}$ & 19.47 & 50.1 & 16.9 & -0.63 & 2.349 & 1.79 \\
\hline & & $\mathrm{Zr}$ & 17.08 & 60.24 & 26.1 & -0.44 & 2.212 & 1.61 \\
\hline & & $\mathrm{Nb}$ & 10.55 & 47.98 & 31.15 & -0.33 & 2.147 & 1.05 \\
\hline & & Mo & 9.91 & 55.28 & 39.63 & -0.06 & 2.078 & 0.11 \\
\hline & & Tc & 8.46 & 49.59 & 45.13 & -0.01 & 2.031 & -0.07 \\
\hline & & $\mathrm{Ru}$ & 14.39 & 51.86 & 48.14 & -0.04 & 2.007 & 0.04 \\
\hline & & $\mathrm{Rh}$ & 26.14 & 48.32 & 48.03 & -0.01 & 2.033 & 0.03 \\
\hline & & $\mathrm{Pd}$ & 41.09 & 42.28 & 58.86 & -0.49 & 2.034 & 0.13 \\
\hline & & $\mathrm{Ag}$ & 92.72 & 27.55 & 37.11 & -0.26 & 2.15 & 0.26 \\
\hline & & La & $a$ & 47.85 & $a$ & $a$ & 2.509 & $a$ \\
\hline & & $\mathrm{Hf}$ & 18.46 & 67.36 & 22.95 & -0.5 & 2.183 & 1.82 \\
\hline & & $\mathrm{Ta}$ & 21.83 & 55.85 & 37.39 & -0.39 & 2.133 & 1.39 \\
\hline & & W & 13.48 & 59.03 & 34.93 & -0.26 & 2.065 & 0.5 \\
\hline & & $\mathrm{Re}$ & 11.56 & 54.75 & 40.97 & -0.13 & 2.035 & 0.17 \\
\hline & & Os & 16.69 & 64.85 & 43.46 & -0.12 & 2.012 & 0.24 \\
\hline & & Ir & 27.5 & 59.76 & 46.01 & 0 & 2.012 & 0.04 \\
\hline & & $\mathrm{Pt}$ & 37.6 & 60.02 & 49.91 & -0.02 & 2.024 & 0 \\
\hline & & $\mathrm{Au}$ & 76.66 & 48.18 & 47.33 & -0.08 & 2.066 & 0.08 \\
\hline \multirow[t]{26}{*}{$\mathrm{C} 4 \mathrm{H} 9$} & \multirow[t]{26}{*}{ B3LYP } & Sc & 13.33 & 50.28 & 20.56 & -0.55 & 2.166 & 1.63 \\
\hline & & $\mathrm{Ti}$ & 13.64 & 45.91 & 32.01 & -0.3 & 2.062 & 1.23 \\
\hline & & $\mathrm{V}$ & 0.36 & 37.77 & 43.33 & -0.31 & 1.978 & 0.35 \\
\hline & & $\mathrm{Cr}$ & 9.64 & 42.81 & 64.3 & 0.21 & 1.947 & -0.06 \\
\hline & & $\mathrm{Mn}$ & & & & & & \\
\hline & & $\mathrm{Fe}$ & 11.15 & 36.26 & 48.64 & 0.05 & 1.873 & 0.19 \\
\hline & & Co & 14.56 & 34.53 & 45.17 & -0.03 & 1.84 & 0.23 \\
\hline & & $\mathrm{Ni}$ & 38 & 33.08 & 43.58 & -0.13 & 1.889 & 0.31 \\
\hline & & $\mathrm{Cu}$ & 89.97 & 37.94 & 36.2 & -0.28 & 1.983 & 0.28 \\
\hline & & $Y$ & 19.3 & 47.03 & 17.33 & -0.63 & 2.354 & 1.77 \\
\hline & & $\mathrm{Zr}$ & 15.94 & 56.52 & 27.11 & -0.44 & 2.226 & 1.61 \\
\hline & & $\mathrm{Nb}$ & 6.85 & 46.52 & 33.98 & -0.18 & 2.157 & 0.74 \\
\hline & & Mo & 5.96 & 51.45 & 42.8 & 0.01 & 2.111 & -0.13 \\
\hline & & Tc & 6.76 & 46.67 & 46.34 & -0.03 & 2.039 & -0.09 \\
\hline & & $\mathrm{Ru}$ & 12.42 & 49.87 & 49.67 & 0 & 2.022 & 0 \\
\hline & & $\mathrm{Rh}$ & 24.24 & 46 & 49.2 & -0.06 & 2.025 & 0.02 \\
\hline & & $\mathrm{Pd}$ & 39.59 & 39.4 & 49.05 & -0.02 & 2.049 & 0.13 \\
\hline & & $\mathrm{Ag}$ & 92.62 & 25.53 & 39.35 & -0.22 & 2.187 & 0.22 \\
\hline & & La & a & 44.41 & a & a & 2.517 & $a$ \\
\hline & & $\mathrm{Hf}$ & 17.52 & 63.77 & 23.81 & -0.5 & 2.199 & 1.82 \\
\hline & & $\mathrm{Ta}$ & 10.29 & 52.92 & 28.82 & -0.28 & 2.138 & 1.16 \\
\hline & & W & 11.51 & 54.87 & 41.91 & -0.16 & 2.085 & 0.66 \\
\hline & & $\mathrm{Re}$ & 6.34 & 53.38 & 43.73 & 0.05 & 2.046 & -0.05 \\
\hline & & Os & 15.31 & 61.72 & 44.43 & -0.09 & 2.038 & 0.21 \\
\hline & & Ir & 24.32 & 56.91 & 47.31 & 0.02 & 2.027 & 0.02 \\
\hline & & $\mathrm{Pt}$ & 35.9 & 57.26 & 50.78 & -0.01 & 2.044 & -0.01 \\
\hline
\end{tabular}




\begin{tabular}{|c|c|c|c|c|c|c|c|c|}
\hline & & $\mathrm{Au}$ & 76.06 & 46.6 & 49.23 & -0.04 & 2.1 & 0.04 \\
\hline \multirow[t]{27}{*}{$\mathrm{CH} 2 \mathrm{~F}$} & \multirow[t]{27}{*}{ B3LYP } & Sc & 21.81 & 59.2 & 18.86 & -0.58 & 2.195 & 1.62 \\
\hline & & $\mathrm{Ti}$ & 19.25 & 49.89 & 28.54 & -0.36 & 2.085 & 1.26 \\
\hline & & $\mathrm{V}$ & 11.89 & 0 & 24.47 & 0.23 & 2.022 & 0.6 \\
\hline & & $\mathrm{Cr}$ & 12.51 & 43.79 & 39.96 & -0.10 & 1.964 & -0.16 \\
\hline & & $\mathrm{Mn}$ & 16.71 & 37.77 & 38.28 & -0.14 & 1.928 & 0.29 \\
\hline & & $\mathrm{Fe}$ & 17.27 & 39.3 & 42.06 & -0.12 & 1.855 & 0.28 \\
\hline & & Co & 25.76 & 41.05 & 39.65 & -0.18 & 1.845 & 0.34 \\
\hline & & $\mathrm{Ni}$ & 41.46 & 44.52 & 39.8 & -0.18 & 1.833 & 0.36 \\
\hline & & $\mathrm{Cu}$ & 90.09 & 47.37 & 32.32 & -0.34 & 1.947 & 0.34 \\
\hline & & $Y$ & 24.7 & 59.2 & 16.7 & -0.63 & 2.355 & 1.76 \\
\hline & & $\mathrm{Zr}$ & 20.22 & 63.24 & 24.32 & -0.46 & 2.253 & 1.61 \\
\hline & & $\mathrm{Nb}$ & 18.07 & 48.71 & 30.87 & -0.30 & 2.179 & 0.96 \\
\hline & & Mo & 12.84 & 55.51 & 36.65 & -0.19 & 2.124 & 0.12 \\
\hline & & Tc & 13.42 & 47.51 & 41.02 & -0.11 & 2.057 & 0.02 \\
\hline & & $\mathrm{Ru}$ & 17.82 & 56.71 & 45.44 & -0.08 & 1.979 & 0.03 \\
\hline & & $\mathrm{Rh}$ & 27.43 & 57.54 & 45.6 & -0.11 & 1.978 & 0.06 \\
\hline & & $\mathrm{Pd}$ & 39.02 & 50.54 & 44.65 & -0.09 & 1.986 & 0.18 \\
\hline & & $\mathrm{Ag}$ & 93.23 & 35.02 & 34.47 & -0.30 & 2.141 & 0.29 \\
\hline & & La & $a$ & 55.51 & $a$ & $a$ & 2.546 & $a$ \\
\hline & & $\mathrm{Hf}$ & 21.67 & 70.81 & 21.65 & -0.51 & 2.229 & 1.84 \\
\hline & & $\mathrm{Ta}$ & 18.81 & 55.81 & 28.94 & -0.36 & 2.154 & 1.32 \\
\hline & & W & 16.46 & 59.02 & 32.69 & -0.26 & 2.121 & 0.51 \\
\hline & & $\mathrm{Re}$ & 15.32 & 51.96 & 37.58 & -0.21 & 2.065 & 0.23 \\
\hline & & Os & 19.35 & 70.65 & 41.97 & -0.17 & 1.988 & 0.26 \\
\hline & & Ir & 28.73 & 67.33 & 44.38 & -0.14 & 1.995 & 0.07 \\
\hline & & $\mathrm{Pt}$ & 39.02 & 67.9 & 47.65 & -0.08 & 1.982 & 0.05 \\
\hline & & $\mathrm{Au}$ & 77.15 & 54.23 & 45.53 & -0.10 & 2.042 & 0.1 \\
\hline \multirow[t]{22}{*}{$\mathrm{BH} 2$} & \multirow[t]{22}{*}{ B3LYP } & Sc & 33.2 & 52.42 & 27.1 & -0.78 & 2.414 & 1.48 \\
\hline & & $\mathrm{Ti}$ & 27 & 45.29 & 40.1 & -0.2 & 2.262 & 1.16 \\
\hline & & V & 13.3 & 33.33 & 50.3 & -0.13 & 2.158 & 0.53 \\
\hline & & $\mathrm{Cr}$ & 17.9 & 39.24 & 51.9 & -0.06 & 2.139 & -0.26 \\
\hline & & $\mathrm{Mn}$ & & & & & & \\
\hline & & $\mathrm{Fe}$ & 32.4 & 58.12 & 62.3 & -0.03 & 1.802 & 0.03 \\
\hline & & Co & 33.7 & 54.16 & 60.2 & -0.05 & 1.802 & 0.15 \\
\hline & & $\mathrm{Ni}$ & 49.6 & 56.9 & 55.7 & -0.05 & 1.836 & 0.17 \\
\hline & & $\mathrm{Cu}$ & 93.6 & 62.02 & 44.2 & -0.17 & 1.965 & 0.17 \\
\hline & & $Y$ & 33.4 & 53.14 & 21.5 & -0.56 & 2.601 & 1.76 \\
\hline & & $\mathrm{Zr}$ & 26.8 & 58.98 & 34.4 & -0.31 & 2.444 & 1.48 \\
\hline & & $\mathrm{Nb}$ & 24.1 & 47.54 & 43.2 & -0.23 & 2.315 & 0.83 \\
\hline & & Mo & 19.1 & 52.71 & 48.3 & 0.04 & 2.268 & -0.04 \\
\hline & & Tc & 8 & 83.28 & 71.5 & -0.05 & 1.926 & -0.15 \\
\hline & & $\mathrm{Ru}$ & 14.5 & 84.03 & 77.7 & 0.18 & 1.966 & -0.22 \\
\hline & & $\mathrm{Rh}$ & 33.9 & 81.33 & 63 & 0.14 & 1.917 & -0.14 \\
\hline & & $\mathrm{Pd}$ & 53.3 & 74 & 59.6 & 0.03 & 1.939 & 0.03 \\
\hline & & $\mathrm{Ag}$ & 93.8 & 52.18 & 46.3 & -0.12 & 2.119 & 0.12 \\
\hline & & $\mathrm{La}$ & $a$ & 49.81 & $a$ & $a$ & 2.768 & $a$ \\
\hline & & $\mathrm{Hf}$ & 26.7 & 65.23 & 30.7 & -0.39 & 2.424 & 1.71 \\
\hline & & $\mathrm{Ta}$ & 24 & 53.21 & 39.7 & -0.28 & 2.316 & 1.17 \\
\hline & & W & 23.1 & 55.86 & 43.8 & -0.06 & 2.279 & 0.36 \\
\hline
\end{tabular}




\begin{tabular}{|c|c|c|c|c|c|c|c|c|}
\hline & & $\mathrm{Re}$ & 21.9 & 88.01 & 59.9 & -0.06 & 1.942 & 0.06 \\
\hline & & Os & 24 & 93.98 & 60.8 & 0 & 1.925 & 0.1 \\
\hline & & Ir & 35.1 & 95.48 & 62 & 0.09 & 1.936 & -0.11 \\
\hline & & $\mathrm{Pt}$ & 50 & 98.5 & 53.8 & 0.14 & 1.928 & -0.09 \\
\hline & & $\mathrm{Au}$ & 18.3 & 82 & 57.9 & 0.03 & 2.005 & -0.03 \\
\hline $\mathrm{F}$ & B3LYP & Sc & 10.91 & 153.37 & 6.8 & -0.77 & 1.853 & 1.81 \\
\hline & & $\mathrm{Ti}$ & 16.02 & 139.43 & 10.49 & -0.56 & 1.765 & 1.45 \\
\hline & & $\mathrm{V}$ & 12.48 & 120.99 & 12.74 & -0.61 & 1.735 & 0.87 \\
\hline & & $\mathrm{Cr}$ & 11.06 & 123.46 & 15.73 & -0.41 & 1.696 & 0.13 \\
\hline & & $\mathrm{Mn}$ & 10.44 & 92.7 & 16.4 & -0.44 & 1.677 & 0.33 \\
\hline & & $\mathrm{Fe}$ & 17.32 & 91.7 & 17.51 & -0.52 & 1.695 & 0.63 \\
\hline & & Co & 22.97 & 86.68 & 16.9 & -0.6 & 1.723 & 0.69 \\
\hline & & $\mathrm{Ni}$ & 38.64 & 80.51 & 16.7 & -0.63 & 1.725 & 0.74 \\
\hline & & $\mathrm{Cu}$ & 92.99 & 89.45 & 9.43 & -0.8 & 1.794 & 0.8 \\
\hline & & $Y$ & 12.99 & 152.8 & 6.02 & -0.75 & 2.015 & 1.94 \\
\hline & & $\mathrm{Zr}$ & 16.1 & 153.95 & 9.2 & -0.63 & 1.928 & 1.84 \\
\hline & & $\mathrm{Nb}$ & 14.63 & 131.21 & 11.81 & -0.54 & 1.892 & 1.27 \\
\hline & & Mo & 11.93 & 129.31 & 15.01 & -0.48 & 1.859 & 0.54 \\
\hline & & Tc & 9.13 & 108.39 & 17.24 & -0.47 & 1.838 & 0.48 \\
\hline & & $\mathrm{Ru}$ & 17.43 & 93.29 & 19.65 & -0.51 & 1.866 & 0.5 \\
\hline & & $\mathrm{Rh}$ & 26.06 & 81.75 & 20.51 & -0.56 & 1.901 & 0.46 \\
\hline & & $\mathrm{Pd}$ & 46.47 & 68.86 & 17.9 & -0.62 & 1.939 & 0.57 \\
\hline & & $\mathrm{Ag}$ & 96.4 & 73.53 & 9.23 & -0.81 & 2.044 & 0.81 \\
\hline & & La & $a$ & 146.07 & $a$ & $a$ & 2.174 & $a$ \\
\hline & & $\mathrm{Hf}$ & 16.27 & 164.67 & 8.68 & -0.64 & 1.896 & 1.99 \\
\hline & & $\mathrm{Ta}$ & 15.24 & 141.71 & 11.14 & -0.57 & 1.874 & 1.57 \\
\hline & & W & 14.48 & 137.58 & 14.05 & -0.49 & 1.85 & 0.86 \\
\hline & & $\operatorname{Re}$ & 11.4 & 118.1 & 16.14 & -0.48 & 1.84 & 0.66 \\
\hline & & Os & 16.03 & 107.74 & 18.41 & -0.52 & 1.878 & 0.67 \\
\hline & & Ir & 25.09 & 89.34 & 20.52 & -0.54 & 1.899 & 0.49 \\
\hline & & $\mathrm{Pt}$ & 39.97 & 74.61 & 22.45 & -0.53 & 1.914 & 0.48 \\
\hline & & $\mathrm{Au}$ & 96.4 & 62.75 & 17.66 & -0.81 & 1.982 & 0.81 \\
\hline $\mathrm{NH} 2$ & B3LYP & Sc & 16.89 & 100.79 & 9.56 & -0.62 & 1.972 & 1.74 \\
\hline & & $\mathrm{Ti}$ & 18.91 & 90.53 & 16.21 & -0.37 & 1.865 & 1.31 \\
\hline & & V & 15.94 & 75.66 & 19.84 & -0.26 & 1.814 & 0.65 \\
\hline & & $\mathrm{Cr}$ & 12.37 & 78.23 & 24.39 & -0.14 & 1.774 & -0.10 \\
\hline & & $\mathrm{Mn}$ & 22.06 & 70.3 & 23.63 & -0.18 & 1.738 & 0.24 \\
\hline & & $\mathrm{Fe}$ & 19.93 & 59.41 & 26.55 & -0.22 & 1.726 & 0.43 \\
\hline & & Co & 18.54 & 54.83 & 29.34 & -0.34 & 1.767 & 0.51 \\
\hline & & $\mathrm{Ni}$ & 32.23 & 56.47 & 29.11 & -0.34 & 1.744 & 0.54 \\
\hline & & $\mathrm{Cu}$ & 91.94 & 50.2 & 15.38 & -0.57 & 1.801 & 0.57 \\
\hline & & $Y$ & 16.8 & 97.52 & 8.06 & -0.69 & 2.149 & 1.92 \\
\hline & & $\mathrm{Zr}$ & 19.07 & 103.4 & 14.27 & -0.47 & 1.749 & 1.69 \\
\hline & & $\mathrm{Nb}$ & 17.57 & 89.98 & 18.47 & -0.29 & 1.963 & 1.00 \\
\hline & & Mo & 14.07 & 87.85 & 23.46 & -0.21 & 1.924 & 0.31 \\
\hline & & Tc & 16.9 & 74.63 & 25.96 & -0.19 & 1.883 & 0.23 \\
\hline & & $\mathrm{Ru}$ & 19.05 & 65.48 & 31.04 & -0.21 & 1.886 & 0.25 \\
\hline & & $\mathrm{Rh}$ & 22.66 & 57.47 & 34.92 & -0.27 & 1.942 & 0.24 \\
\hline & & $\mathrm{Pd}$ & 39.13 & 49.39 & 33.79 & -0.29 & 1.943 & 0.36 \\
\hline & & $\mathrm{Ag}$ & 95.17 & 31.42 & 15.21 & -0.53 & 2.042 & 0.53 \\
\hline
\end{tabular}




$\begin{array}{cccccccc} & \mathrm{La} & \mathrm{a} & 89.92 & \mathrm{a} & \mathrm{a} & 2.308 & \mathrm{a} \\ \mathrm{Hf} & 19.03 & 112.97 & 13.24 & -0.52 & 1.727 & 1.89 \\ \mathrm{Ta} & 17.98 & 99.38 & 17.34 & -0.34 & 1.95 & 1.32 \\ \mathrm{~W} & 16.22 & 97.84 & 21.61 & -0.26 & 1.918 & 0.66 \\ \mathrm{Re} & 13.7 & 82.79 & 24.96 & -0.21 & 1.888 & 0.42 \\ \mathrm{Os} & 19.11 & 83.25 & 28.2 & -0.22 & 1.888 & 0.45 \\ \mathrm{OH} \mathrm{Ir} & 22.02 & 70.75 & 32.9 & -0.26 & 1.942 & 0.27 \\ \mathrm{Pt} & 33.62 & 64.91 & 36.39 & -0.22 & 1.915 & 0.25 \\ & \mathrm{Au} & 80.65 & 37.66 & 28.43 & -0.32 & 1.961 & 0.32 \\ \mathrm{Sc} & 7.47 & 117.12 & 6.19 & -0.65 & 2.179 & 1.76 \\ \mathrm{Ti} & 21.47 & 115.45 & 10.16 & -0.46 & 1.763 & 1.39 \\ \mathrm{~V} & 11.19 & 120.14 & 15.89 & -0.34 & 1.739 & 0.74 \\ \mathrm{Cr} & 8.17 & 99.69 & 20.43 & -0.26 & 1.714 & 0.02 \\ \mathrm{Mn} & 18.92 & 78.06 & 20.19 & -0.34 & 1.743 & 0.43 \\ \mathrm{Fe} & 17.25 & 71.83 & 22.49 & -0.38 & 1.705 & 0.54 \\ \mathrm{Co} & 19.77 & 69.33 & 22.14 & -0.46 & 1.718 & 0.64 \\ \mathrm{Ni} & 42.66 & 61.91 & 22.37 & -0.50 & 1.755 & 0.66 \\ \mathrm{Cu} & 90.95 & 65.92 & 14.61 & -0.71 & 1.809 & 0.71 \\ \mathrm{Y} & 15.56 & 124.71 & 5.69 & -0.73 & 2.023 & 1.95 \\ \mathrm{Zr} & 20.7 & 129.18 & 8.91 & -0.56 & 1.924 & 1.80 \\ \mathrm{Nb} & 14.2 & 109.01 & 14.66 & -0.41 & 1.898 & 1.17 \\ \mathrm{Mo} & 11.85 & 105.72 & 18.96 & -0.35 & 1.873 & 0.44 \\ \mathrm{Tc} & 12.75 & 88.59 & 21.61 & -0.34 & 1.867 & 0.34 \\ \mathrm{Ru} & 16.79 & 74.09 & 25.27 & -0.37 & 1.878 & 0.39 \\ \mathrm{Rh} & 23.27 & 65.38 & 26.85 & -0.42 & 1.908 & 0.39 \\ \mathrm{Pd} & 47.6 & 51.27 & 25.16 & -0.46 & 1.971 & 0.47 \\ \mathrm{Ag} & 95.2 & 48.61 & 15.61 & -0.69 & 2.069 & 0.69 \\ \mathrm{La} & \mathrm{a} & 126.82 & \mathrm{a} & \mathrm{a} & 1.863 & \mathrm{a} \\ \mathrm{Hf} & 19.33 & 140.01 & 8.6 & -0.59 & 1.895 & 1.96 \\ \mathrm{Ta} & 14.32 & 120.14 & 13.56 & -0.44 & 1.875 & 1.48 \\ \mathrm{~W} & 14.45 & 115.88 & 16.98 & -0.37 & 1.855 & 0.79 \\ \mathrm{Re} & 12.24 & 98.16 & 20.28 & -0.35 & 1.852 & 0.55 \\ \mathrm{Os} & 17.25 & 89.92 & 22.49 & -0.40 & 1.705 & 0.57 \\ \mathrm{Ir} & 21.58 & 76.02 & 26.18 & -0.40 & 1.893 & 0.41 \\ \mathrm{Pt} & 29.47 & 60.6 & 41.25 & -0.38 & 1.949 & 0.37 \\ \mathrm{Au} & 82.21 & 47.55 & 25.74 & -0.49 & 1.999 & 0.49\end{array}$

${ }^{a} \mathrm{NBO}$ analysis is not available for La.

Table S2. Bond enthalpy ( $\left.\mathrm{D}_{\mathrm{e}}, \mathrm{kcal} / \mathrm{mol}\right)$ for cationic, valency-saturated compounds $\mathrm{H}_{n} \mathrm{M}^{+}$-L for different ligands $\mathrm{L}$ and metals $\mathrm{M}$, at B3LYP level of theory with $\mathrm{LACV} 3 \mathrm{P}++* *$ basis set.

\begin{tabular}{ccc} 
Ligand & Metal & $\mathbf{D}_{\mathbf{e}} \mathbf{( \mathbf { k c a l } / \mathbf { m o l } )}$ \\
\hline $\mathrm{CH}_{3}$ & $\mathrm{Sc}$ & \\
& $\mathrm{Ti}$ & 56.9 \\
& $\mathrm{~V}$ & 51.7 \\
& $\mathrm{Cr}$ & 50.9 \\
& $\mathrm{Mn}$ & 43
\end{tabular}




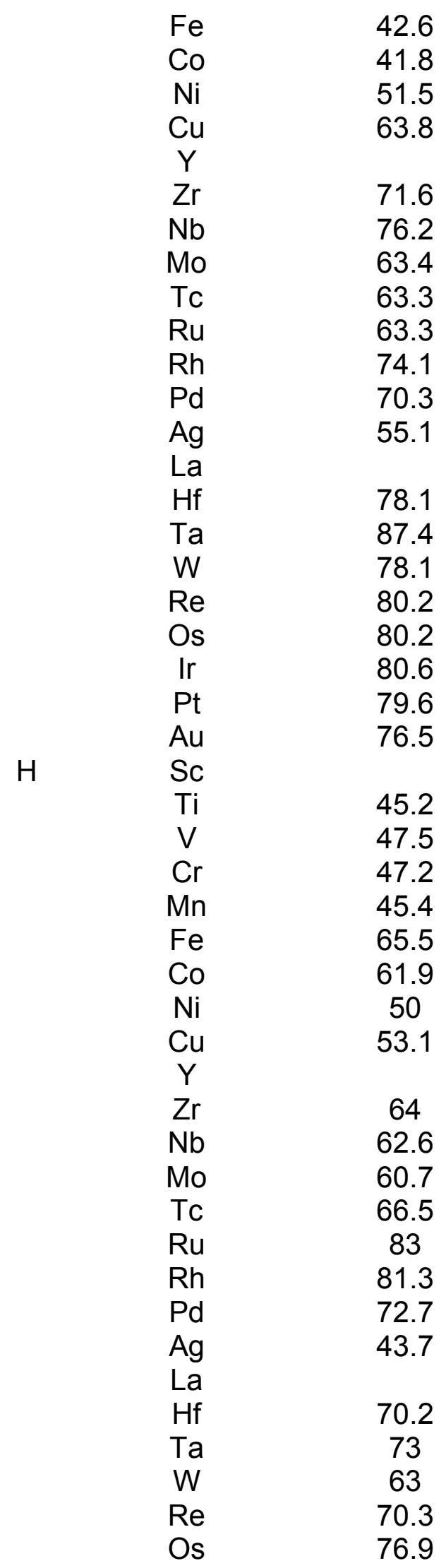


Ir

$\mathrm{Au}$

84

84.7

76.6 
Selected Cartesian Coordinates and Total Energies

\begin{tabular}{|c|c|c|c|}
\hline Jobname & Total E(har & $\mathrm{ZPE}(\mathrm{kcal} / \mathrm{mol})$ & $X Y Z$ \\
\hline 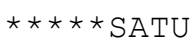 & RATED HYDRIDES & 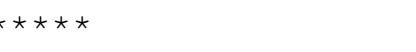 & \\
\hline Row 1 & & & \\
\hline ScH3_pyr. & -48.1 & 375919.376 & \\
\hline $\mathrm{Sc}$ & 0.0212707600 & -0.0041414149 & 0.0000000000 \\
\hline H1 & 0.0212707600 & 1.7999385558 & 0.0000000000 \\
\hline $\mathrm{H} 2$ & -0.4850462870 & -0.8076015209 & 1.5338837788 \\
\hline H3 & -0.4850462870 & -0.8076015209 & -1.5338837788 \\
\hline TiH4_C3v_ & -60.3 & 14.559 & \\
\hline $\mathrm{Ti}$ & -0.0000739107 & 0.0000412090 & -0.0000764348 \\
\hline H1 & 0.0015270877 & -0.0007647312 & 1.6878310281 \\
\hline $\mathrm{H} 2$ & 1.5910969178 & -0.0006617191 & -0.5632414544 \\
\hline H3 & -0.7959184959 & -1.3785225316 & -0.5614472391 \\
\hline H4 & -0.7963673648 & 1.3779884360 & -0.5619007952 \\
\hline VH5 & -74.1 & $58668 \quad 18.958$ & \\
\hline V1 & 0.0001711180 & 0.0321473209 & 0.0000000000 \\
\hline $\mathrm{H} 2$ & -0.0083544743 & 1.6778352020 & 0.0000000000 \\
\hline H3 & 0.9524943259 & -0.8184722807 & -0.9604387070 \\
\hline $\mathrm{H} 4$ & 0.9524943259 & -0.8184722807 & 0.9604387070 \\
\hline H5 & -0.9526419614 & -0.8329434707 & 0.9442725555 \\
\hline H 6 & -0.9526419614 & -0.8329434707 & -0.9442725555 \\
\hline BE_CrH6.0 & $1-89.7118900$ & 569 & \\
\hline $\mathrm{Cr}^{-}$ & 0.0000000000 & 0.0000000000 & 1.0000000000 \\
\hline $\mathrm{H} 1$ & 0.8257488091 & 0.0000000000 & -0.2715416601 \\
\hline $\mathrm{H} 2$ & -0.6253593051 & 0.5391623446 & -0.2714569934 \\
\hline H3 & -0.3621058565 & -0.9765100755 & -0.1016992800 \\
\hline H4 & 1.5313308135 & 0.2857590174 & 1.1794882643 \\
\hline H5 & -0.9754722539 & 1.2145797509 & 1.1789638061 \\
\hline H6 & -0.5221437004 & -1.4009388051 & 1.4729516517 \\
\hline $\mathrm{CrH} 6 \_\mathrm{C} 3 v_{-}$ & i_r $\quad-89.7$ & $10073 \quad 35.271$ & \\
\hline $\operatorname{Cr} 1$ & $\overline{0} .0000000000$ & 0.0000000000 & 0.0000000000 \\
\hline $\mathrm{x} 2$ & 0.0000000000 & 0.0000000000 & 1.0000007970 \\
\hline H3 & 0.9089112067 & 0.0000000000 & 1.1972403016 \\
\hline H4 & -0.4443711264 & -0.7698844821 & 1.2121535001 \\
\hline H5 & -0.4345003721 & 0.7870882236 & 1.2046599007 \\
\hline H 6 & 0.7621882363 & -1.3344354700 & 0.2745698009 \\
\hline $\mathrm{H} 7$ & -1.5344052061 & 0.0111102150 & 0.2872537786 \\
\hline H 8 & 0.7790625211 & 1.3272457024 & 0.2617593179 \\
\hline $\mathrm{MnH} 5 \_\mathrm{C} 5 \mathrm{v}$ & -106.748335 & 27.276 & \\
\hline $\mathrm{Mn}-$ & 0.0048998526 & -0.0029412504 & 0.0035753874 \\
\hline $\mathrm{H} 1$ & -0.0185297764 & 0.0113088725 & 1.5014146905 \\
\hline H2 & 1.4299449484 & 0.0097808553 & -0.4593008965 \\
\hline H3 & 0.6512266851 & -1.2737789634 & -0.4566762799 \\
\hline H4 & -0.2488366445 & -1.2764062292 & 0.7515087596 \\
\hline H5 & 1.0157730373 & 0.8087663875 & 0.7549059508 \\
\hline FeH4_Td_r & -125.691866 & 17.901 & \\
\hline & -0.0000613237 & 0.0000462135 & -0.0000323761 \\
\hline
\end{tabular}




\begin{tabular}{|c|c|c|c|}
\hline $\mathrm{H} 1$ & 0.0011818804 & -0.0008365812 & 1.4722546834 \\
\hline $\mathrm{H} 2$ & 1.3883706323 & -0.0008939539 & -0.4897030844 \\
\hline H3 & -0.6934616762 & 1.2019151690 & -0.4905614802 \\
\hline H4 & -0.6927403042 & -1.2028974947 & -0.4899827461 \\
\hline $\mathrm{COH} 3 \_\mathrm{C} 3 \mathrm{v} . \mathrm{C}$ & $02-146.781629$ & 11.973 & \\
\hline $\mathrm{Co}-$ & 0.0000000000 & 0.0000000000 & 0.0000000000 \\
\hline H1 & 0.0000000000 & 0.0000000000 & 1.4325390714 \\
\hline $\mathrm{H} 2$ & 1.4318505772 & 0.0000000000 & -0.0444085100 \\
\hline H3 & -0.0486184406 & -1.4310249203 & -0.0444085100 \\
\hline $\mathrm{NiH} 2 \_\mathrm{C} 2 \mathrm{v} . \mathrm{C}$ & $02-170.446480$ & 7.183 & \\
\hline $\mathrm{Ni}-$ & 0.0000000000 & 0.0000000000 & 0.0000000000 \\
\hline $\mathrm{H} 1$ & 0.0000000000 & 0.0000000000 & 1.4172607198 \\
\hline $\mathrm{H} 2$ & 1.4127222586 & 0.0000000000 & 0.1133303483 \\
\hline $\mathrm{CuH} 1 \_\mathrm{C} 2 \mathrm{v} \cdot \mathrm{C}$ & $01-196$. & 7388332.691 & \\
\hline $\mathrm{Cu}-$ & 0.0000000000 & 0.0000000000 & 0.0000000000 \\
\hline $\mathrm{H} 1$ & 0.0000000000 & 0.0000000000 & 1.4728120298 \\
\hline ow 2 & & & \\
\hline YH3_pyr.03 & $3 \quad-39.681982$ & 8.407 & \\
\hline $\mathrm{Y}-\mathrm{-}-\mathrm{x}$ & 0.0159999680 & -0.0042142756 & 0.0000000000 \\
\hline $\mathrm{H} 1$ & 0.0159999680 & 1.9729354217 & 0.0000000000 \\
\hline $\mathrm{H} 2$ & -0.7137230381 & -0.8005853721 & 1.6560248599 \\
\hline H3 & -0.7137230381 & -0.8005853721 & -1.6560248599 \\
\hline ZrH4_Td.03 & $3-48.893139$ & 13.676 & \\
\hline Zr & 0.0000000000 & 0.0000000000 & 0.0000000000 \\
\hline $\mathrm{H} 1$ & 0.0000000000 & 0.0000000000 & 1.8501223129 \\
\hline $\mathrm{H} 2$ & 1.7442888245 & 0.0000000000 & -0.6167731102 \\
\hline H3 & -0.8722943143 & -1.5105118777 & -0.6167731102 \\
\hline H 4 & -0.8722943143 & 1.5105118777 & -0.6167731102 \\
\hline $\mathrm{NbH} 5$ & $.193486 \quad 19.11$ & 524 & \\
\hline $\mathrm{Nb} 1$ & 0.0000828352 & 0.0171534918 & 0.0000000000 \\
\hline $\mathrm{H} 2$ & -0.0104759054 & 1.7995351931 & 0.0000000000 \\
\hline H3 & 1.0742305659 & -0.8385435568 & -1.0794661622 \\
\hline $\mathrm{H} 4$ & 1.0742305659 & -0.8385435568 & 1.0794661622 \\
\hline H5 & -1.0728106943 & -0.8518715888 & 1.0682962411 \\
\hline H 6 & -1.0728106943 & -0.8518715888 & -1.0682962411 \\
\hline MoH 6 & $.006863 \quad 27.92$ & 676 & \\
\hline Mo1 & -0.0020944623 & -0.0014940503 & -0.0032989394 \\
\hline $\mathrm{H} 2$ & 0.0224169852 & 0.0168694975 & 1.6392018625 \\
\hline H3 & 1.4524799443 & 0.0146906655 & 0.7605435799 \\
\hline H4 & 0.4684304521 & 1.3744949755 & 0.7610683576 \\
\hline H5 & 1.2343183466 & -0.8838754181 & -0.7466045460 \\
\hline H 6 & -0.4560853726 & 1.4483561567 & -0.7460273484 \\
\hline $\mathrm{H} 7$ & -1.2233220190 & -0.8848992874 & 0.7630428188 \\
\hline $\mathrm{TCH} 5 \_\mathrm{C} 5 \mathrm{v}$ & -82 & $64705 \quad 25.910$ & \\
\hline $\mathrm{TC}$ & -0.0039267079 & 0.0027647113 & -0.0024540483 \\
\hline $\mathrm{H} 1$ & 0.1027668619 & -0.0718758289 & 1.5954481131 \\
\hline H2 & 1.4913408362 & -0.0737891283 & -0.5759176044 \\
\hline H3 & 0.5782061408 & -1.3768146387 & -0.5754432621 \\
\hline H4 & -0.2817003762 & -1.3781003228 & 0.7633973157 \\
\hline
\end{tabular}




\begin{tabular}{|c|c|c|c|}
\hline H5 & 1.1984059796 & 0.7345363586 & 0.7651839782 \\
\hline RuH4_C3v. & -96.2 & $214544 \quad 18.299$ & \\
\hline $\mathrm{Ru}-$ & 0.0000014387 & -0.0000008188 & 0.0000988407 \\
\hline $\mathrm{H} 1$ & -0.0002291851 & 0.0002110940 & 1.5324409745 \\
\hline $\mathrm{H} 2$ & 1.4183634080 & -0.0000483870 & 0.6618625249 \\
\hline 3 & -0.7092133439 & -1.2281482892 & 0.6621397141 \\
\hline 14 & -0.7089385473 & 1.2284430232 & 0.6618813750 \\
\hline RhH3 C3v. & $.02-111.276434$ & 412.780 & \\
\hline $\mathrm{Rh}-0.0$ & 0000000000 & 0.0000000000 & 0.0000000000 \\
\hline 0.0 & 0000000000 & 0.0000000000 & 1.5219189039 \\
\hline 1.5 & 5129311032 & 0.0000000000 & 0.1651563714 \\
\hline 0.1 & 1483876239 & -1.5056366215 & 0.1651563714 \\
\hline $\mathrm{PdH} 2 \mathrm{C} 2 \mathrm{v}$. & $.01-127.910817$ & 76.895 & \\
\hline $\mathrm{Pd}-$ & 0.0000000000 & 0.0000000000 & 0.0000000000 \\
\hline H1 & 0.0000000000 & 0.0000000000 & 1.5221060354 \\
\hline $\mathrm{H} 2$ & 1.4408781386 & 0.0000000000 & 0.4905883943 \\
\hline $\mathrm{AgH}_{1} \mathrm{C} 2 \mathrm{v}$. & $.01-146$. & .3501362 .476 & \\
\hline $\mathrm{Ag}-$ & 0.0000000000 & 0.0000000000 & 0.0000000000 \\
\hline H1 & 0.0000000000 & 0.0000000000 & 1.6285622715 \\
\hline ow 3 & & & \\
\hline LaH3.01 & -32.966143 & 7.66385 & \\
\hline La1 & 0.0000000000 & 0.0000000000 & 0.0164017135 \\
\hline H2 & -1.9837346641 & 0.0000000000 & -0.7535375875 \\
\hline H3 & 0.9918673321 & -1.7179646135 & -0.7535375875 \\
\hline H4 & 0.9918673321 & 1.7179646135 & -0.7535375875 \\
\hline $\mathrm{BE} \_\mathrm{H} f \mathrm{H} 4 . \mathrm{C}$ & out -51.2367321 & 18615 & \\
\hline $\mathrm{Hf}^{-}$ & 0.0000000000 & 0.0000000000 & 0.0000000000 \\
\hline $\mathrm{H} 1$ & 0.0000000000 & 0.0000000000 & 1.8324016427 \\
\hline $\mathrm{H} 2$ & 1.7275667429 & 0.0000000000 & -0.6109126910 \\
\hline H3 & -0.8639899215 & 1.4960482594 & -0.6107735244 \\
\hline H4 & -0.8638919258 & -1.4960672965 & -0.6108130647 \\
\hline TaH5.01 & -60.712522 & 19.96286 & \\
\hline $\mathrm{Tal}$ & -0.0000235278 & 0.0078474152 & 0.0000000000 \\
\hline H2 & -0.0087826509 & 1.7913792333 & 0.0000000000 \\
\hline H3 & 1.0994402683 & -0.7939990267 & -1.0975434491 \\
\hline H4 & 1.0994402683 & -0.7939990267 & 1.0975434491 \\
\hline H5 & -1.0929368124 & -0.8061650843 & 1.0948422357 \\
\hline H 6 & -1.0929368124 & -0.8061650843 & -1.0948422357 \\
\hline WH 6.01 & -71.309271 & 28.68659 & \\
\hline W1 & 0.0000055349 & 0.0000610798 & 0.0000872156 \\
\hline $\mathrm{H} 2$ & -0.0004755191 & -0.0009093762 & 1.6625882958 \\
\hline H3 & 1.4823935727 & -0.0038610124 & 0.7528338767 \\
\hline $\mathrm{H} 4$ & 0.4652138364 & 1.4075312559 & 0.7530201780 \\
\hline H5 & 1.1514712870 & -0.9294993363 & -0.8506405818 \\
\hline H 6 & -0.5189189772 & 1.3873620747 & -0.8486462371 \\
\hline H7 & -1.2832848377 & -0.9240369538 & 0.6430382728 \\
\hline $\mathrm{ReH} 5 \_\mathrm{C} 5 \mathrm{v}$ & -81.982766 & 24.565 & \\
\hline & 0.0007860348 & 0.0012802357 & 0.0025447168 \\
\hline
\end{tabular}




\begin{tabular}{|c|c|c|c|}
\hline $\mathrm{H} 1$ & -0.4557682215 & 0.1501013382 & 1.5539089521 \\
\hline $\mathrm{H} 2$ & 1.6071522437 & 0.0116000496 & -0.2383613263 \\
\hline H3 & 0.7063419688 & -1.4144381134 & -0.3649722767 \\
\hline $\mathrm{H} 4$ & -0.5691353557 & -1.3284555813 & 0.7405835182 \\
\hline H5 & 0.8890667390 & 0.9763482955 & 0.9499349664 \\
\hline OsH4.01 & -93.382476 & 19.043 & \\
\hline $\mathrm{H} 1$ & 0.9246217931 & 0.9246217931 & -0.9246217931 \\
\hline$s 2$ & 0.0000000000 & 0.0000000000 & 0.0000000000 \\
\hline 3 & 0.9246217931 & -0.9246217931 & 0.9246217931 \\
\hline 5 & -0.9246217931 & 0.9246217931 & 0.9246217931 \\
\hline 6 & -0.9246217931 & -0.9246217931 & -0.9246217931 \\
\hline $\operatorname{IrH} 3.01$ & -106.468337 & 13.30 & \\
\hline $\operatorname{Ir} 1$ & 0.0002188314 & 0.0000705838 & -0.1347611022 \\
\hline H2 & -0.6172181289 & 1.1268308648 & 0.7281708552 \\
\hline 3 & -0.6725161923 & -1.0956703744 & 0.7266347918 \\
\hline H4 & 1.2841244953 & -0.0308970937 & 0.7295539989 \\
\hline PtH2.01 & -120.343494 & 8.153 & \\
\hline Pt1 & 0.0000000000 & 0.0000000000 & -0.0113246847 \\
\hline $\mathrm{H} 2$ & 0.0000000000 & 1.0418905457 & 1.0953858670 \\
\hline H3 & 0.0000000000 & -1.0418905457 & 53858670 \\
\hline AuH1_C2v & $.01 \quad-136.0$ & 592163.249 & \\
\hline $\mathrm{Au} \quad-$ & 0.0000000000 & 0.0000000000 & 0.0000000000 \\
\hline $\mathrm{H} 1$ & 0.0000000000 & 0.0000000000 & 1.5422806020 \\
\hline \multirow{2}{*}{\multicolumn{4}{|c|}{$\star \star \star \star * t-$ butyl-substituted-SATURATED HYDRIDES $* \star \star \star * *$}} \\
\hline & & & \\
\hline \multicolumn{2}{|c|}{ ScH2tBu_agcorr_r.01 } & \multicolumn{2}{|l|}{$-205.499565 \quad 85.260$} \\
\hline $\mathrm{Sc} 1$ & $-.01 \overline{3} 6988529$ & -.0255731154 & -.0006931984 \\
\hline $\mathrm{C} 2$ & .0008563907 & .0044704142 & 2.1654607865 \\
\hline $\mathrm{C} 3$ & 1.4652852179 & .0662874381 & 2.6114027703 \\
\hline $\mathrm{C} 4$ & -.7735710067 & 1.2494155861 & 2.6100410407 \\
\hline H5 & -1.6152856880 & -.4909903928 & -.7569271941 \\
\hline H6 & 1.0036978294 & 1.2709205140 & -.7978882044 \\
\hline $\mathrm{C} 7$ & -.6804800535 & -1.2812127353 & 2.6444513591 \\
\hline H8 & -.1518188717 & -2.1851651158 & 2.3087068317 \\
\hline H9 & -.7118915646 & -1.3396284715 & 3.7449988891 \\
\hline $\mathrm{H} 10$ & -1.7164020703 & -1.3585233916 & 2.2947365162 \\
\hline $\mathrm{H} 11$ & 1.9765587653 & .9613859521 & 2.2384536738 \\
\hline $\mathrm{H} 12$ & 1.5561662052 & .0844563701 & 3.7100272003 \\
\hline $\mathrm{H} 13$ & 2.0427218469 & -.8073403031 & 2.2752969236 \\
\hline $\mathrm{H} 14$ & -1.8162916997 & 1.2325651183 & 2.2685292445 \\
\hline H1 5 & -.8083027112 & 1.3357027453 & 3.7087013183 \\
\hline H1 6 & -.3158134237 & 2.1759269624 & 2.2413695051 \\
\hline \multicolumn{3}{|c|}{ TiH3tBu.01 -217.702799} & \\
\hline $\operatorname{Ti} 1$ & 0.0000000000 & 0.0000000000 & 0.0000000000 \\
\hline C2 & 0.0000000000 & 0.0000000000 & 2.0618340608 \\
\hline H3 & 1.5922695850 & 0.0000000000 & -0.6102968621 \\
\hline $\mathrm{H} 4$ & -0.7938698313 & -1.3789158723 & -0.6134281209 \\
\hline H5 & -0.7942392311 & 1.3791365611 & -0.6125027621 \\
\hline C 6 & -0.7534908882 & -1.2603733574 & 2.5021752423 \\
\hline C7 & 1.4687569425 & -0.0268873126 & 2.5018287791 \\
\hline $\mathrm{C} 8$ & -0.7104977404 & 1.2819892791 & 2.5102725434 \\
\hline
\end{tabular}




\begin{tabular}{|c|c|c|c|}
\hline H9 & -0.7822858699 & -1.3156504057 & 009795103 \\
\hline $\mathrm{H} 10$ & -1.7918241579 & -1.2720823896 & 2.1543027072 \\
\hline H11 & -0.2753736283 & -2.1798451777 & 2.1485970938 \\
\hline $\mathrm{H} 12$ & -0.7336860247 & 1.3339714180 & 3.6093920752 \\
\hline $\mathrm{H} 13$ & -0.2037973278 & 2.1867370969 & 2.1583495241 \\
\hline $\mathrm{H} 14$ & -1.7489173066 & 1.3286736666 & 2.1659374244 \\
\hline H1 5 & 1.5305183626 & -0.0361724900 & 3.6006266225 \\
\hline H1 6 & 1.9983543750 & -0.9166088788 & 2.1451299440 \\
\hline $\mathrm{H} 17$ & 2.0264480457 & 0.8500769076 & 2.1572774904 \\
\hline VH4tBu_r & -231.502352 & 97.270 & \\
\hline V1 & .0405877038 & -.0712466532 & -.1296138227 \\
\hline $\mathrm{H} 2$ & .0621346254 & -.0486241924 & 1.4470140103 \\
\hline H3 & 1.1788164382 & .6805783417 & .7633195316 \\
\hline H4 & .7562044333 & -1.3276393852 & -.9464175004 \\
\hline H5 & -1.1673468091 & -.6612903464 & .7923801830 \\
\hline $\mathrm{C} 6$ & -.8044085905 & 1.3792135860 & -1.1770882983 \\
\hline $\mathrm{C} 7$ & .5075475777 & 1.6070593786 & -1.9225395196 \\
\hline $\mathrm{C} 8$ & -1.6712444257 & .3505661845 & -1.8972287871 \\
\hline C9 & -1.4995439027 & 2.5961539893 & -.6095758434 \\
\hline $\mathrm{H} 10$ & .3443168624 & 1.7238171903 & -3.0010619041 \\
\hline $\mathrm{H} 11$ & 1.0636379168 & 2.4624087632 & -1.5415722765 \\
\hline $\mathrm{H} 12$ & 1.1797243099 & .7187118885 & -1.8559283498 \\
\hline $\mathrm{H} 13$ & -1.8833510369 & 3.2463307466 & -1.4092765655 \\
\hline $\mathrm{H} 14$ & -2.3497447725 & 2.3109338285 & .0155477867 \\
\hline H1 5 & -.8163003034 & 3.1949578836 & -.0016217026 \\
\hline H1 6 & -1.7069733591 & .5372030969 & -2.9777094295 \\
\hline $\mathrm{H} 17$ & -1.2383601097 & -.6760171118 & -1.8259206366 \\
\hline $\mathrm{H} 18$ & -2.6839624866 & .3028385102 & -1.4996255946 \\
\hline CrH5tBu_r & -246.960671 & 108.705 & \\
\hline $\operatorname{Cr} 1$ & .0105674504 & .0060548563 & -.0008670869 \\
\hline C2 & .0157452700 & .0057074229 & 2.4705819885 \\
\hline H3 & 1.4312249730 & .0172353191 & .5781962919 \\
\hline H4 & .3907554115 & -1.3688155762 & .5731638425 \\
\hline H5 & -1.1800053253 & -.8032050768 & .5198451468 \\
\hline H6 & -1.1455130384 & .8655503257 & .5427550377 \\
\hline $\mathrm{H} 7$ & .4689983418 & 1.3725509061 & .5201770939 \\
\hline $\mathrm{C} 8$ & 1.2127252568 & -.8084125369 & 2.9255197991 \\
\hline C9 & .1200372301 & 1.4553676904 & 2.9033876789 \\
\hline C10 & -1.2937564475 & -.6238010913 & 2.9050103177 \\
\hline $\mathrm{H} 11$ & 1.1633173558 & -1.8426256539 & 2.5845221478 \\
\hline $\mathrm{H} 12$ & 2.1566156495 & -.3760978528 & 2.5929200099 \\
\hline $\mathrm{H} 13$ & 1.2167934196 & -.8171009091 & 4.0282250871 \\
\hline $\mathrm{H} 14$ & 1.0509804146 & 1.9183213273 & 2.5756448060 \\
\hline H1 5 & -.7157855949 & 2.0552147486 & 2.5443366974 \\
\hline $\mathrm{H} 16$ & .1034624488 & 1.4793733924 & 4.0058345167 \\
\hline $\mathrm{H} 17$ & -2.1587804047 & -.0628219316 & 2.5520822654 \\
\hline $\mathrm{H} 18$ & -1.3847469843 & -1.6580821818 & 2.5727703829 \\
\hline $\mathrm{H} 19$ & -1.3182913057 & -.6223908492 & 4.0076818787 \\
\hline
\end{tabular}

Mn: not found

$\begin{array}{lrrr}\text { FeH3tBu.02 } & -283.021875 & 95.949 & \\ \text { Fe1 } & 0.0566482979 & -0.0290250177 & 0.0087598405 \\ \text { C2 } & -0.0474367019 & 0.0328846398 & 1.8780148564 \\ \text { H3 } & 1.4464084644 & -0.1825985886 & -0.4869405621\end{array}$




\begin{tabular}{|c|c|c|c|}
\hline H4 & -0.7146814406 & -1.1532540309 & -0.5760879371 \\
\hline H5 & -0.4401696070 & 1.1489506975 & -0.7605601324 \\
\hline C6 & -1.5111355196 & 0.1371199288 & 2.2754247768 \\
\hline $\mathrm{C} 7$ & 0.5643974854 & -1.3572366536 & 1.9445742105 \\
\hline $\mathrm{C} 8$ & 0.8329648227 & 1.1716057929 & 2.3679062009 \\
\hline H9 & -1.6265723767 & -0.0322636628 & 3.3556270185 \\
\hline $\mathrm{H} 10$ & -1.9168284705 & 1.1274132511 & 2.0530137851 \\
\hline $\mathrm{H} 11$ & -2.1261537773 & -0.6116342272 & 1.7671141955 \\
\hline $\mathrm{H} 12$ & 0.9586032904 & 1.1142272931 & 3.4587630438 \\
\hline $\mathrm{H} 13$ & 1.8343451571 & 1.1311293675 & 1.9286409510 \\
\hline $\mathrm{H} 14$ & 0.3928851334 & 2.1455060002 & 2.1385483288 \\
\hline $\mathrm{H} 15$ & 1.5245587436 & -1.3733447967 & 2.4635012175 \\
\hline H1 6 & -0.1116454905 & -2.0969860942 & 2.3767676872 \\
\hline H1 7 & 0.7874081111 & -1.7355417785 & 0.9143121206 \\
\hline $\mathrm{CoH} 2 \mathrm{tBu} . \mathrm{OI}$ & -304.102203 & 90.941 & \\
\hline $\operatorname{Col}$ & 0.0000000000 & 0.0000000000 & 0.0000000000 \\
\hline C2 & 0.0000000000 & 0.0000000000 & 2.3483954744 \\
\hline C3 & 1.1928596473 & 0.0000000000 & 1.4003519453 \\
\hline $\mathrm{C} 4$ & 2.2816382185 & 1.0073270335 & 1.6568542932 \\
\hline $\mathrm{C} 5$ & 1.6157437384 & -1.4046733788 & 0.9902683626 \\
\hline H6 & 1.8626891811 & 2.0106821010 & 1.7661174022 \\
\hline $\mathrm{H} 7$ & 2.8430493637 & 0.7776654922 & 2.5754612552 \\
\hline H 8 & 2.9940233616 & 1.0281803609 & 0.8286253016 \\
\hline H9 & 0.8792124694 & -1.8460093796 & 0.2670007708 \\
\hline $\mathrm{H} 10$ & 1.6329754490 & -2.1164193905 & 1.8197587573 \\
\hline $\mathrm{H} 11$ & 2.5792527870 & -1.4133078246 & 0.4741005929 \\
\hline $\mathrm{H} 14$ & -0.9458151996 & -0.2492488781 & 1.7973234372 \\
\hline H1 5 & -0.1713750387 & 0.9813095807 & 2.7985018339 \\
\hline H1 6 & 0.0614801204 & -0.7619568319 & 3.1297375173 \\
\hline H12 & -0.4937371063 & 1.3558426349 & -0.1621730844 \\
\hline $\mathrm{H} 13$ & 0.7374612261 & 0.2616408320 & -1.2239707913 \\
\hline NiHtBu_r & -327.758949 & 83.567 & \\
\hline $\mathrm{Nil}$ & -.0203052915 & -.0008105466 & -.0959226656 \\
\hline C2 & .0411147525 & .0006866482 & 1.7922879551 \\
\hline C3 & 1.5182509734 & .0006301930 & 2.1912044755 \\
\hline $\mathrm{C} 4$ & -.6689860897 & 1.2709685061 & 2.2610099951 \\
\hline $\mathrm{C} 5$ & -.6694438498 & -1.2685154981 & 2.2630181884 \\
\hline H 6 & 2.0502216763 & .8883378468 & 1.8321438205 \\
\hline H7 & 1.5974962336 & .0025674900 & 3.2878223950 \\
\hline H 8 & 2.0492319015 & -.8888634221 & 1.8352225038 \\
\hline H9 & -1.4512984360 & -.0022086183 & -.2725638045 \\
\hline $\mathrm{H} 10$ & -.2087639642 & -2.1724606613 & 1.8552074814 \\
\hline $\mathrm{H} 11$ & -.6226228417 & -1.3435802859 & 3.3620143350 \\
\hline $\mathrm{H} 12$ & -1.7224054764 & -1.2680230975 & 1.9766228529 \\
\hline H13 & -1.7216398176 & 1.2711907749 & 1.9734168290 \\
\hline $\mathrm{H} 14$ & -.6233570851 & 1.3468976220 & 3.3599894995 \\
\hline H1 5 & -.2070584872 & 2.1742157489 & 1.8530230238 \\
\hline CutBu_new & -354.042376 & 78.584 & \\
\hline $\mathrm{Cu} 1-$ & 0.0029023182 & -0.0001152691 & 0.0081910957 \\
\hline C2 & 0.0043950023 & 0.0001280429 & 1.9918390310 \\
\hline C3 & 1.4571785839 & 0.0034838821 & 2.4727001702 \\
\hline $\mathrm{C} 4$ & -0.7243546513 & 1.2560690140 & 2.4741758021 \\
\hline $\mathrm{C} 5$ & -0.7187311474 & -1.2591497750 & 2.4741183873 \\
\hline H 6 & -1.7650029350 & 1.2857436345 & 2.1414652854 \\
\hline
\end{tabular}




$\begin{array}{lr}\text { H7 } & -0.7339490940 \\ \text { H8 } & -0.2346116796 \\ \text { H9 } & -0.2245161764 \\ \text { H10 } & -0.7288153372 \\ \text { H11 } & -1.7591239871 \\ \text { H12 } & 2.0029902119 \\ \text { H13 } & 1.4786371239 \\ \text { H14 } & 2.0072705083\end{array}$

Row 2

YH2tBu_agcorr_r.01

Y1 -0.0004230241

C2 $\quad-0.0009855126$

C3 1.4560115504

C4 $\quad-0.7260825709$

H5 -1.5403492520

H6 $\quad 0.1144638332$

C7 $\quad-0.7264692481$

H8 $\quad-0.2295607266$

H9 $\quad-0.7678008617$

H10 $\quad-1.7624372791$

H11 2.0092880564

H12 1.5244774722

H13 2.0134905490

H14 -1.7740405198

H15 -0.7431883747

H16 -0.2507255285

ZrH3tBu_r $\quad-206.206940 \quad 90.504$

$\begin{array}{lr}\text { Zr1 } & -0.0000685579 \\ \text { C2 } & 0.0002478651 \\ \text { H3 } & 1.7742414288 \\ \text { H4 } & -0.8873483300 \\ \text { H5 } & -0.8874814504 \\ \text { C6 } & -1.4599592622 \\ \text { C7 } & 0.7287022428 \\ \text { C8 } & 0.7509145370 \\ \text { H9 } & -1.5143883437 \\ \text { H10 } & -1.9996804531 \\ \text { H11 } & -2.0143945570 \\ \text { H12 } & 0.2579391680 \\ \text { H13 } & 0.8009104516 \\ \text { H14 } & 1.7856047493 \\ \text { H15 } & 0.7798304350 \\ \text { H16 } & 0.2186446321 \\ \text { H17 } & 1.7621888854\end{array}$

1.2736045355

2.1744624701

$-2.1752355722$

$-1.2764937897$

$-1.2938562682$

0.8896401919

0.0032388919

$-0.8798872227$

$-196.98623687 .150$

$-0.0003576449$

$-0.0001912762$

0.0018402456

1.2624966330

$-1.0398697492$

1.8539418040

$-1.2623142650$

$-2.1847163500$

$-1.3139421196$

$-1.3102049402$

0.8879144288

0.0022440530

$-0.8868858840$

1.2878626741

1. 3268052680

2.1825891689
3.5781995271

2.1405971817

2.1409183566

3.5781394914

2.1409563753

2.1390205137

3.5767036812

2.1386009573
-0.0000638970
2.3537291463
2.8368322680
2.8358478238
-0.7122637988
-0.7127510817
2.8359472927
2.5046115977
3.9378636910
2.4805012675
2.4975172964
3.9386470513
2.5042485757
2.5102687793
3.9377561585
2.4750773244

0.0051539350

2.2310933382

$-0.5723821041$

$-0.5735290207$

$-0.5736688663$

2.7028307087

2.6837723070

2.6681672549

3. 8025247538

2.3551979828

2. 3678547379

2. 3232238930

3.7661287430

2. 3065541861

3. 7824471069

2. 3513584748

2. 3205211610

0.0022398624

1.7292909201

0.5918939350

$-0.9343051930$

0.7739427368

$-0.9479382459$

$-1.1666861645$

0.2182241497

$-2.2168072204$ 


\begin{tabular}{|c|c|c|c|}
\hline $\mathrm{H} 11$ & -2.8388357203 & 2.6547816773 & -1.5214177932 \\
\hline $\mathrm{H} 12$ & -2.7727028435 & 0.9376807993 & -1.9186995190 \\
\hline $\mathrm{H} 13$ & -3.0280721197 & 1.4498113430 & -0.2466179263 \\
\hline $\mathrm{H} 14$ & -0.1661175097 & 2.9184352930 & -2.5568868273 \\
\hline 15 & 0.9191826504 & 1.6306076468 & -2.0669231183 \\
\hline 6 & -0.5097203318 & 1.2478696966 & -3.0363533894 \\
\hline 17 & 0.0817191436 & 3.4096397181 & -0.0780568358 \\
\hline 18 & -1.2684098076 & 2.7894564237 & 0.9166334742 \\
\hline 19 & 0.2869601143 & 1.9680489771 & 0.8654816297 \\
\hline MotB & $03-228$ & 326153108.655 & \\
\hline Mo1 & 0.0027685185 & -0.0022521058 & 0.0021584393 \\
\hline H2 & -0.0054810360 & 0.0126485393 & 1.6551325459 \\
\hline H3 & 1.4748120595 & -0.0096662361 & 0.7660335313 \\
\hline $\mathrm{H} 4$ & 0.5034847304 & 1.3937770197 & 0.7385748861 \\
\hline H5 & 1.2573563440 & -0.8043155151 & -0.8053705460 \\
\hline H 6 & -0.4047187762 & 1.4664937069 & -0.7657286256 \\
\hline C7 & -1.5991084152 & -1.2822227098 & 0.5024189395 \\
\hline $\mathrm{C} 8$ & -2.0271846257 & -1.2609087234 & -0.9660464491 \\
\hline $\mathrm{C} 9$ & -0.7195819385 & -2.4789007829 & 0.8441436528 \\
\hline C10 & -2.6968081357 & -0.9676727315 & 1.4964582946 \\
\hline $\mathrm{H} 11$ & -2.2550788949 & -2.2575075740 & -1.3574469599 \\
\hline H12 & -2.8754918866 & -0.5998185659 & -1.1449913056 \\
\hline $\mathrm{H} 13$ & -1.2085927843 & -0.9077769308 & -1.6501915153 \\
\hline $\mathrm{H} 14$ & -3.4823862496 & -1.7373572537 & 1.4831150979 \\
\hline H1 5 & -2.2971594475 & -0.9150011314 & 2.5118628922 \\
\hline H1 6 & -3.1789442690 & -0.0108920177 & 1.2742833071 \\
\hline $\mathrm{H} 17$ & -1.0840274191 & -3.4069120822 & 0.3868249010 \\
\hline H18 & 0.3156727814 & -2.3643590304 & 0.4541145532 \\
\hline $\mathrm{H} 19$ & -0.6374100376 & -2.6139070793 & 1.9220441655 \\
\hline $\mathrm{TCH} 4$ & C5V_r $\quad-240$. & 273330101.320 & \\
\hline $\mathrm{TC}$ & 0.0987666630 & -0.0046003254 & 0.0607540508 \\
\hline $\mathrm{H} 1$ & 0.1677789768 & -0.0372055473 & 1.6866166463 \\
\hline $\mathrm{H} 2$ & 1.5057623239 & -0.0246937769 & -0.7170459295 \\
\hline H3 & 0.9540540946 & -1.3751292352 & 0.1280614227 \\
\hline C4 & -1.6973312050 & -0.0386836634 & -0.9045470570 \\
\hline H5 & 0.9207074624 & 1.3894035145 & 0.0999063836 \\
\hline $\mathrm{C} 7$ & -2.3560893086 & 1.2824684104 & -0.5067130753 \\
\hline $\mathrm{C} 8$ & -1.3222845465 & -0.1380205287 & -2.3768636968 \\
\hline C9 & -2.3998633650 & -1.2700572063 & -0.3346304598 \\
\hline $\mathrm{H} 10$ & -0.4772191467 & 0.5211110020 & -2.6220236110 \\
\hline $\mathrm{H} 11$ & -1.0559586490 & -1.1568135969 & -2.6632902207 \\
\hline $\mathrm{H} 12$ & -2.1555678226 & 0.1863749139 & -3.0140361033 \\
\hline $\mathrm{H} 13$ & -3.3744639942 & -1.4107165249 & -0.8217267379 \\
\hline $\mathrm{H} 14$ & -1.8235673720 & -2.1851937901 & -0.5042119012 \\
\hline H1 5 & -2.5941318292 & -1.1770489792 & 0.7389431713 \\
\hline $\mathrm{H} 16$ & -1.8927399961 & 2.1353027931 & -1.0078515412 \\
\hline $\mathrm{H} 17$ & -3.4205527753 & 1.2742159062 & -0.7759183072 \\
\hline $\mathrm{H} 18$ & -2.3158400877 & 1.4594614799 & 0.5763896413 \\
\hline RuH3 & $03-253.524882$ & 94.647 & \\
\hline Ru1 & -0.0164830033 & 0.0004035250 & 0.0029273066 \\
\hline C2 & 0.0502355327 & -0.0011973291 & 2.0234436528 \\
\hline H3 & 1.3962029210 & -0.0008455713 & -0.7085539615 \\
\hline H4 & -0.7564768609 & -1.2407390425 & -0.6404568719 \\
\hline H5 & -0.7533588519 & 1.2443572103 & -0.6386312568 \\
\hline
\end{tabular}




\begin{tabular}{|c|c|c|c|}
\hline C6 & -1.4062486198 & 0.0019909905 & 2.4830905229 \\
\hline $\mathrm{C} 7$ & 0.7971040259 & -1.2777610130 & 2.4049488361 \\
\hline $\mathrm{C} 8$ & 0.8032595935 & 1.2712087099 & 2.4067869512 \\
\hline H9 & -1.4553114197 & -0.0012812750 & 3.5809368438 \\
\hline $\mathrm{H} 10$ & -1.9405155500 & 0.8930978137 & 2.1411605728 \\
\hline H11 & -1.9460280684 & -0.8837539806 & 2.1357980476 \\
\hline $\mathrm{H} 12$ & 0.8847006999 & 1.3460517895 & 3.5000847193 \\
\hline H13 & 1.8221108405 & 1.2785909409 & 2.0085959649 \\
\hline H1 4 & 0.2868128489 & 2.1715469055 & 2.0614954668 \\
\hline H1 5 & 0.8822603649 & -1.3523334566 & 3.4979766322 \\
\hline H1 6 & 0.2742286270 & -2.1752686441 & 2.0620960880 \\
\hline H1 7 & 1.8144902040 & -1.2913256521 & 2.0031024796 \\
\hline $\mathrm{RhH} 2 \mathrm{tBu}$ & -268.579820 & 89.042 & \\
\hline Rh1 & 0.0917188829 & -0.0201350260 & -0.0691913332 \\
\hline C2 & -0.1587402937 & 0.0099798945 & 2.6919596972 \\
\hline C3 & 1.0294433629 & 0.0362525553 & 1.7249114931 \\
\hline C 4 & 1.9518208604 & 1.2194545269 & 1.9787826020 \\
\hline C5 & 1.7858708582 & -1.2961075527 & 1.7183295239 \\
\hline H 6 & 1.4089208394 & 2.1654982863 & 1.9269931597 \\
\hline $\mathrm{H} 7$ & 2.4031927600 & 1.1450467302 & 2.9805209755 \\
\hline H 8 & 2.7637243992 & 1.2539487044 & 1.2494964977 \\
\hline H9 & 1.1587824521 & -2.1320397437 & 1.3748019944 \\
\hline $\mathrm{H} 10$ & 2.1122402186 & -1.5641989560 & 2.7340991731 \\
\hline H11 & 2.6732809706 & -1.2522055791 & 1.0837226717 \\
\hline H1 4 & -0.6539058404 & 0.9815819493 & 2.7481114050 \\
\hline H1 5 & 0.1707739462 & -0.2582526786 & 3.7066624195 \\
\hline H1 6 & -0.9100449248 & -0.7429922169 & 2.4117953363 \\
\hline H12 & -0.2909067647 & 1.4619251668 & -0.1738173939 \\
\hline $\mathrm{H} 13$ & 1.2672104346 & 0.4077180568 & -0.9576132822 \\
\hline PdHtBu_r & -285.212808 & 83.226 & \\
\hline $\mathrm{Pd} 1$ & 0.0291651896 & -0.0004879779 & 0.0000971063 \\
\hline C2 & -0.0553508743 & 0.0006371385 & 2.0473554297 \\
\hline C3 & 1.4493896267 & 0.0009496354 & 2.3313292832 \\
\hline $\mathrm{C} 4$ & -0.7318490071 & 1.2643719317 & 2.5660229685 \\
\hline C5 & -0.7322703192 & -1.2611748971 & 2.5699767723 \\
\hline H6 & 1.9492073105 & 0.8900631606 & 1.9326001453 \\
\hline H7 & 1.6201964102 & 0.0082907596 & 3.4187942169 \\
\hline H8 & 1.9474498724 & -0.8938792972 & 1.9438201057 \\
\hline H9 & -1.5080652651 & -0.0068210580 & -0.1504178633 \\
\hline $\mathrm{H} 10$ & -0.2746440366 & -2.1695659755 & 2.1723813606 \\
\hline H11 & -0.6450352382 & -1.2962545244 & 3.6679823954 \\
\hline $\mathrm{H} 12$ & -1.7939048153 & -1.2769248270 & 2.3196198072 \\
\hline H13 & -1.7930029313 & 1.2807396008 & 2.3137190706 \\
\hline H1 4 & -0.6465276016 & 1.3016950140 & 3.6641098928 \\
\hline H1 5 & -0.2725433147 & 2.1715016313 & 2.1673906423 \\
\hline AgtBu_new & -303.649521 & 78.585 & \\
\hline $\operatorname{Ag} 1-$ & 0.0031001967 & -0.0000812831 & -0.0550449323 \\
\hline C2 & 0.0041030034 & 0.0000831824 & 2.1318277786 \\
\hline C3 & 1.4598737033 & 0.0034305812 & 2.5869753355 \\
\hline $\mathrm{C} 4$ & -0.7263769712 & 1.2585829811 & 2.5883928518 \\
\hline C5 & -0.7205588943 & -1.2618204188 & 2.5883252048 \\
\hline H 6 & -1.7632438605 & 1.2816500162 & 2.244866900 \\
\hline H7 & -0.7443327144 & 1.2915127296 & 3.692097649 \\
\hline H 8 & -0.2331343614 & 2.1702471798 & 2.243225572 \\
\hline
\end{tabular}




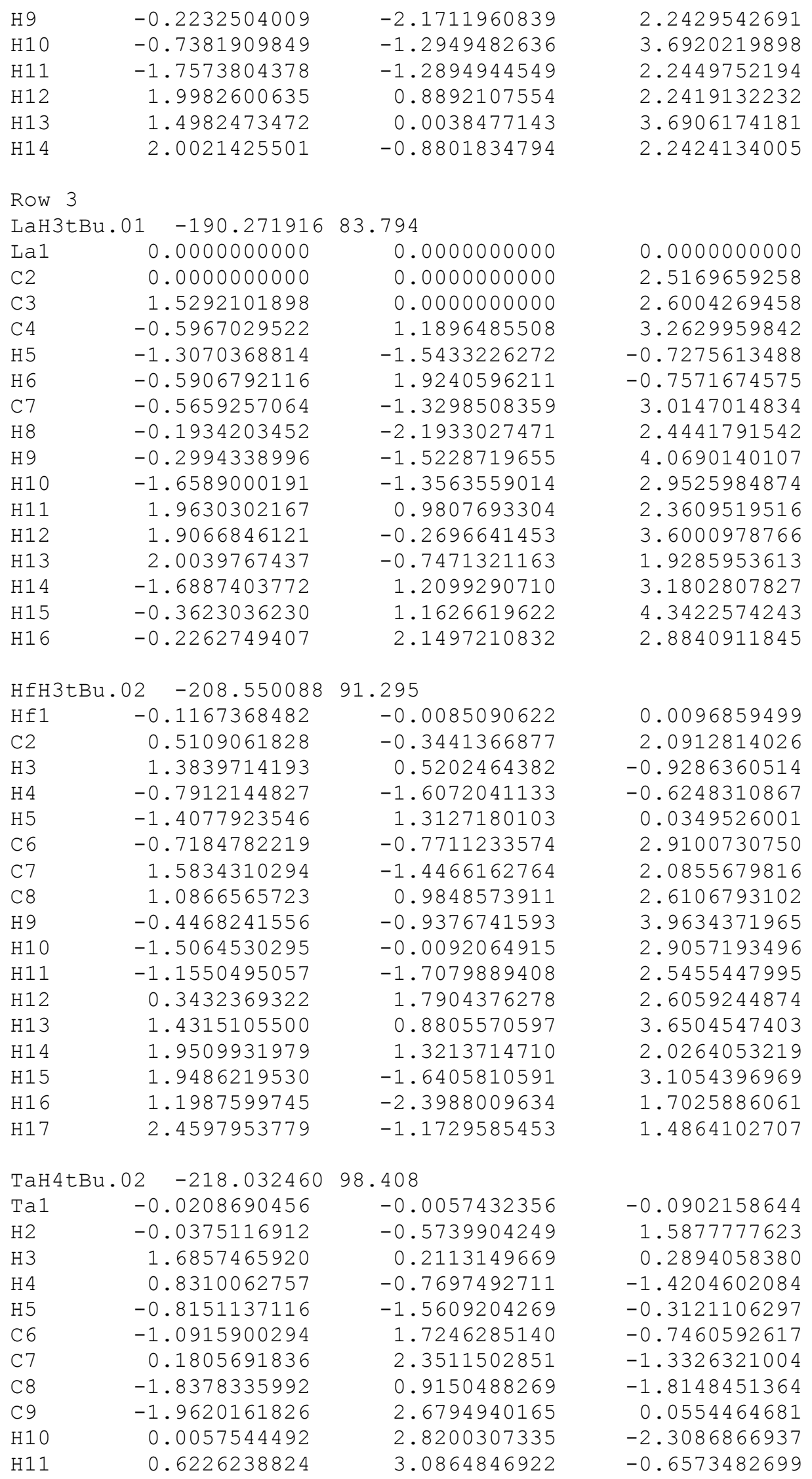




\begin{tabular}{|c|c|c|c|}
\hline $\mathrm{H} 12$ & 0.9817278483 & 1.5994966339 & -1.5416062175 \\
\hline $\mathrm{H} 13$ & -2.3767892637 & 3.4758942148 & -0.5787364509 \\
\hline $\mathrm{H} 14$ & -2.8076695547 & 2.1609470720 & 0.5171065473 \\
\hline H1 5 & -1.3945788764 & 3.1657299519 & 0.8546420991 \\
\hline H1 6 & -1.9118318973 & 1.4484595591 & -2.7702797768 \\
\hline H1 7 & -1.3162513901 & -0.0367989317 & -2.0854541820 \\
\hline H1 8 & -2.8389272934 & 0.6292958684 & -1.4858178583 \\
\hline WH5t & -228.5 & $597626 \quad 107.332$ & \\
\hline W1 & 0.0022355158 & 0.0003035447 & -0.0051163068 \\
\hline $\mathrm{C} 2$ & -0.0234760453 & -0.0127380920 & 2.2251845744 \\
\hline H3 & 1.5669931948 & -0.0070846444 & 0.5684229960 \\
\hline H4 & 0.5751386435 & 1.4576288738 & 0.5655409196 \\
\hline H5 & 0.9932718322 & -1.0383287086 & -0.9305584572 \\
\hline H 6 & -0.5929022537 & 1.3038488035 & -0.9347711772 \\
\hline H7 & -1.3438517213 & -0.9107579587 & 0.5291696948 \\
\hline $\mathrm{C} 8$ & 0.0556270242 & -1.5052916983 & 2.5821302082 \\
\hline C9 & 1.0948240845 & 0.7448150353 & 2.9520924658 \\
\hline C10 & -1.3798499211 & 0.6176625851 & 2.5772559563 \\
\hline H11 & -0.8656652784 & -2.0435527720 & 2.3535192109 \\
\hline $\mathrm{H} 12$ & 0.8840967676 & -2.0060476355 & 2.0615987388 \\
\hline H13 & 0.2603548802 & -1.6294726347 & 3.6544609486 \\
\hline $\mathrm{H} 14$ & 2.0806811699 & 0.3459247220 & 2.7023252833 \\
\hline H1 5 & 1.0916254006 & 1.8079127537 & 2.7004642279 \\
\hline H1 6 & 0.9641825192 & 0.6579612547 & 4.0401912017 \\
\hline $\mathrm{H} 17$ & -1.5351597578 & 1.5712455281 & 2.0529666092 \\
\hline $\mathrm{H} 18$ & -2.2219769119 & -0.0378068234 & 2.3490213293 \\
\hline H19 & -1.4210890077 & 0.8582564697 & 3.6485800778 \\
\hline $\mathrm{ReH} 4$ & $02-239.310422$ & 100.454 & \\
\hline $\operatorname{Re} 1$ & 0.0000000000 & 0.0000000000 & 0.0000000000 \\
\hline H2 & 0.0000000000 & 0.0000000000 & 1.6380661379 \\
\hline H3 & 1. 4586150572 & 0.0000000000 & -0.7534255164 \\
\hline $\mathrm{C} 4$ & -1.7877431232 & -0.6246826608 & -0.7747474583 \\
\hline H5 & 1.1666912276 & -1.0885785720 & 0.3828208704 \\
\hline H 6 & 0.4118140978 & 1.5652918804 & -0.3298109377 \\
\hline $\mathrm{C} 7$ & -2.4405744447 & 0.5053457361 & 0.0026673439 \\
\hline $\mathrm{C} 8$ & -2.0286355283 & -0.6763472991 & -2.2701974875 \\
\hline C9 & -1.7344829253 & -1.9571440608 & -0.0394625985 \\
\hline $\mathrm{H} 10$ & -3.0535226411 & -1.0042171840 & -2.4897299211 \\
\hline H11 & -1.8930016472 & 0.3050135331 & -2.7331479579 \\
\hline $\mathrm{H} 12$ & -1.3447474838 & -1.3797935345 & -2.7530055740 \\
\hline $\mathrm{H} 13$ & -2.6279686845 & -2.1517239243 & 0.5628660867 \\
\hline $\mathrm{H} 14$ & -1.5592542592 & -2.7840149664 & -0.7264661165 \\
\hline H1 5 & -0.8934948971 & -1.9751529754 & 0.7037091787 \\
\hline $\mathrm{H} 16$ & -2.8435779809 & 1.2855708046 & -0.6416618845 \\
\hline H1 7 & -3.1835303666 & 0.1865254612 & 0.7361982726 \\
\hline $\mathrm{H} 18$ & -1.6541720742 & 1.0430735083 & 0.6510315513 \\
\hline OsH3 & $03-250.698970$ & 94.115 & \\
\hline Os 1 & -0.0079776759 & 0.0010619517 & 0.0000012043 \\
\hline C2 & 0.0438928714 & -0.0030785194 & 2.0383084586 \\
\hline H3 & 1.4517815557 & -0.0346160806 & -0.6655784967 \\
\hline $\mathrm{H} 4$ & -0.7938270264 & -1.2557823201 & -0.6151072948 \\
\hline H5 & -0.7285851833 & 1.3003133143 & -0.6065478202 \\
\hline C 6 & -1.4111492814 & 0.0532116181 & 2.5287928555 \\
\hline $\mathrm{C} 7$ & 0.7407229328 & -1.3007069999 & 2.4748995934 \\
\hline
\end{tabular}




\begin{tabular}{|c|c|c|c|}
\hline $\mathrm{C} 8$ & 0.8373483367 & 1.2399969565 & 2.4699514151 \\
\hline H9 & -1.4372458534 & 0.0407254338 & 3.6268586863 \\
\hline $\mathrm{H} 10$ & -1.9174297272 & 0.9680185344 & 2.2068056604 \\
\hline $\mathrm{H} 11$ & -1.9936058194 & -0.8070448679 & 2.1854456248 \\
\hline $\mathrm{H} 12$ & 0.8751406503 & 1.2981457973 & 3.5662212558 \\
\hline $\mathrm{H} 13$ & 1.8714271569 & 1.2100941919 & 2.1144225252 \\
\hline $\mathrm{H} 14$ & 0.3738531694 & 2.1663984851 & 2.1171780565 \\
\hline H1 5 & 0.8066072320 & -1.3402315473 & 3.5706460060 \\
\hline H1 6 & 0.1896051561 & -2.1897996338 & 2.1546294314 \\
\hline H1 7 & 1.7631978499 & -1.3669380119 & 2.0908569234 \\
\hline \multicolumn{4}{|c|}{ IrH2tBu flat } \\
\hline $\operatorname{Ir} 1$ & -0.0063063283 & -0.0121980103 & -0.0012295341 \\
\hline $\mathrm{C} 2$ & 0.0297288181 & 0.0569334303 & 2.7958094081 \\
\hline C3 & 1.1052748936 & 0.0376515600 & 1.6932873824 \\
\hline $\mathrm{C} 4$ & 2.0763938538 & 1.2028719003 & 1.8290737596 \\
\hline $\mathrm{C} 5$ & 1.8374812579 & -1.3166786702 & 1.6545048457 \\
\hline H 6 & 1.5498359347 & 2.1597177550 & 1.8101864443 \\
\hline H7 & 2.6249826212 & 1.1388062555 & 2.7807788688 \\
\hline H8 & 2.8099669900 & 1.2018476358 & 1.0197531163 \\
\hline H9 & 1.1709989243 & -2.1490106099 & 1.3845805477 \\
\hline $\mathrm{H} 10$ & 2.2390762528 & -1.5634692796 & 2.6466782216 \\
\hline $\mathrm{H} 11$ & 2.6712895920 & -1.3030365398 & 0.9497950496 \\
\hline $\mathrm{H} 14$ & -0.4167972351 & 1.0472638866 & 2.9049871782 \\
\hline H1 5 & 0.4687588292 & -0.2231674192 & 3.7629531990 \\
\hline H1 6 & -0.7773557359 & -0.6670126984 & 2.6109331309 \\
\hline $\mathrm{H} 12$ & -0.5881104167 & 1.4171207409 & -0.1853663438 \\
\hline $\mathrm{H} 13$ & 0.9758101181 & 0.2213145161 & -1.1833099747 \\
\hline PtHtBu. 02 & -277.647358 & 83.855 & \\
\hline Pt1 & 0.0082815674 & -0.0007352008 & -0.0004757781 \\
\hline C2 & -0.0265369600 & 0.0020372968 & 2.0427460275 \\
\hline $\mathrm{C} 3$ & 1.4756869541 & 0.0025021698 & 2.3696049162 \\
\hline $\mathrm{C} 4$ & -0.7117857629 & 1.2657648273 & 2.5594377004 \\
\hline C5 & -0.7124729483 & -1.2586516140 & 2.5653194134 \\
\hline H 6 & 1.9856410935 & 0.8897982808 & 1.9806243030 \\
\hline $\mathrm{H} 7$ & 1.6105731349 & 0.0142092559 & 3.4600574322 \\
\hline H8 & 1.9823819720 & -0.8938364066 & 1.9982888922 \\
\hline H9 & -1.4918198598 & -0.0132821647 & -0.3062544571 \\
\hline $\mathrm{H} 10$ & -0.2390011458 & -2.1674535010 & 2.1888475717 \\
\hline $\mathrm{H} 11$ & -0.6566982203 & -1.2781720009 & 3.6638768723 \\
\hline $\mathrm{H} 12$ & -1.7677510790 & -1.2822854658 & 2.2865679624 \\
\hline H13 & -1.7661122912 & 1.2906619839 & 2.2773275500 \\
\hline $\mathrm{H} 14$ & -0.6592786911 & 1.2885913811 & 3.6580925846 \\
\hline H1 5 & -0.2355126727 & 2.1726209060 & 2.1817021591 \\
\hline AutBu_new & -293.362025 & 78.728 & \\
\hline Aul - & 0.0029236074 & -0.0000707436 & -0.0129091047 \\
\hline C2 & 0.0044176828 & -0.0000149587 & 2.0874399223 \\
\hline C3 & 1.4631700401 & 0.0032816008 & 2.5411339171 \\
\hline $\mathrm{C} 4$ & -0.7275510798 & 1.2610624522 & 2.5425800821 \\
\hline C5 & -0.7217898174 & -1.2644080889 & 2.5426083916 \\
\hline H 6 & -1.7634126379 & 1.2799028696 & 2.1992022965 \\
\hline H7 & -0.7391569544 & 1.2825235380 & 3.6431612956 \\
\hline $\mathrm{H} 8$ & -0.2313280952 & 2.1699970108 & 2.1978711704 \\
\hline H9 & -0.2212176444 & -2.1710377795 & 2.1981011227 \\
\hline $\mathrm{H} 10$ & -0.7335000090 & -1.2858037843 & 3.6431904522 \\
\hline
\end{tabular}




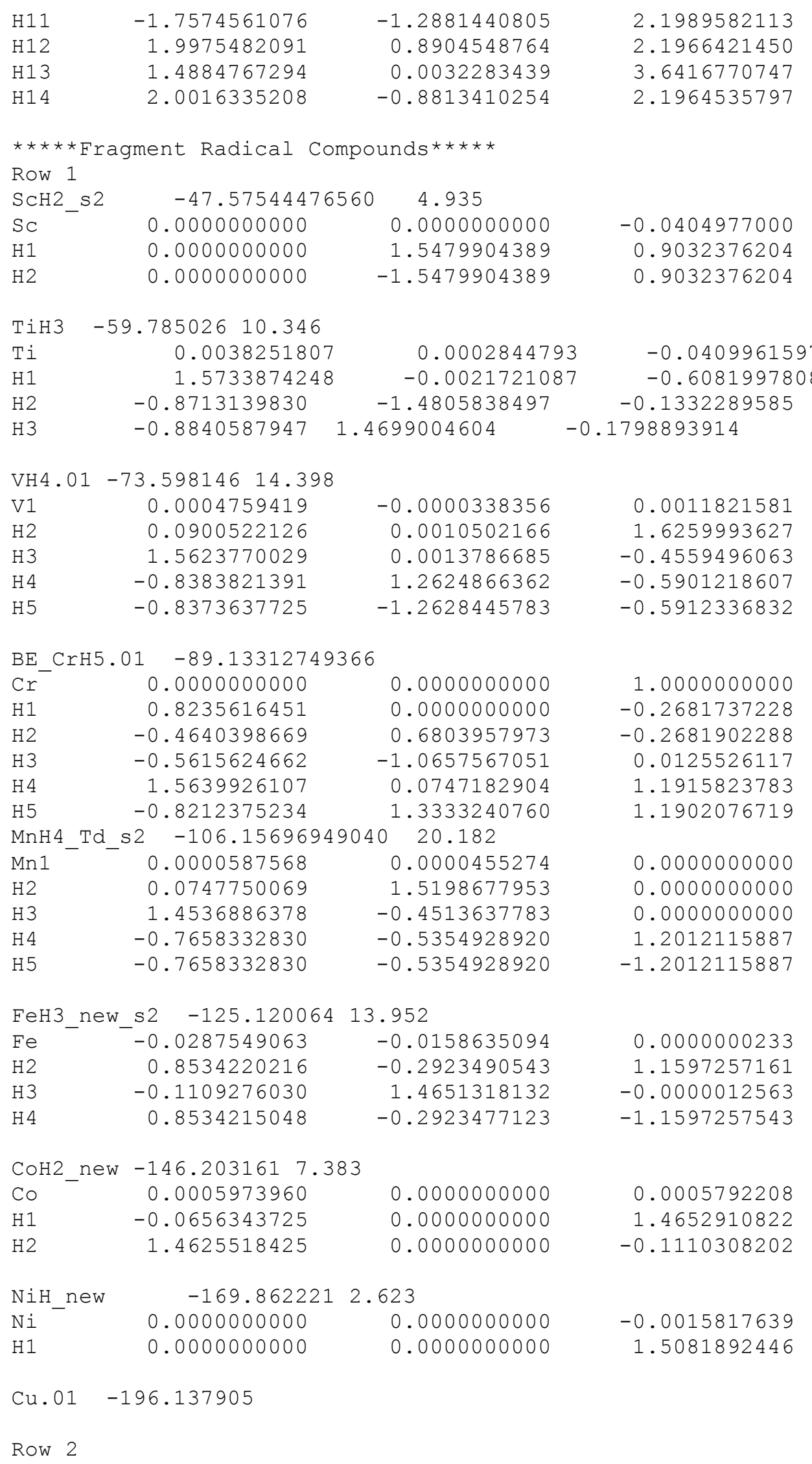




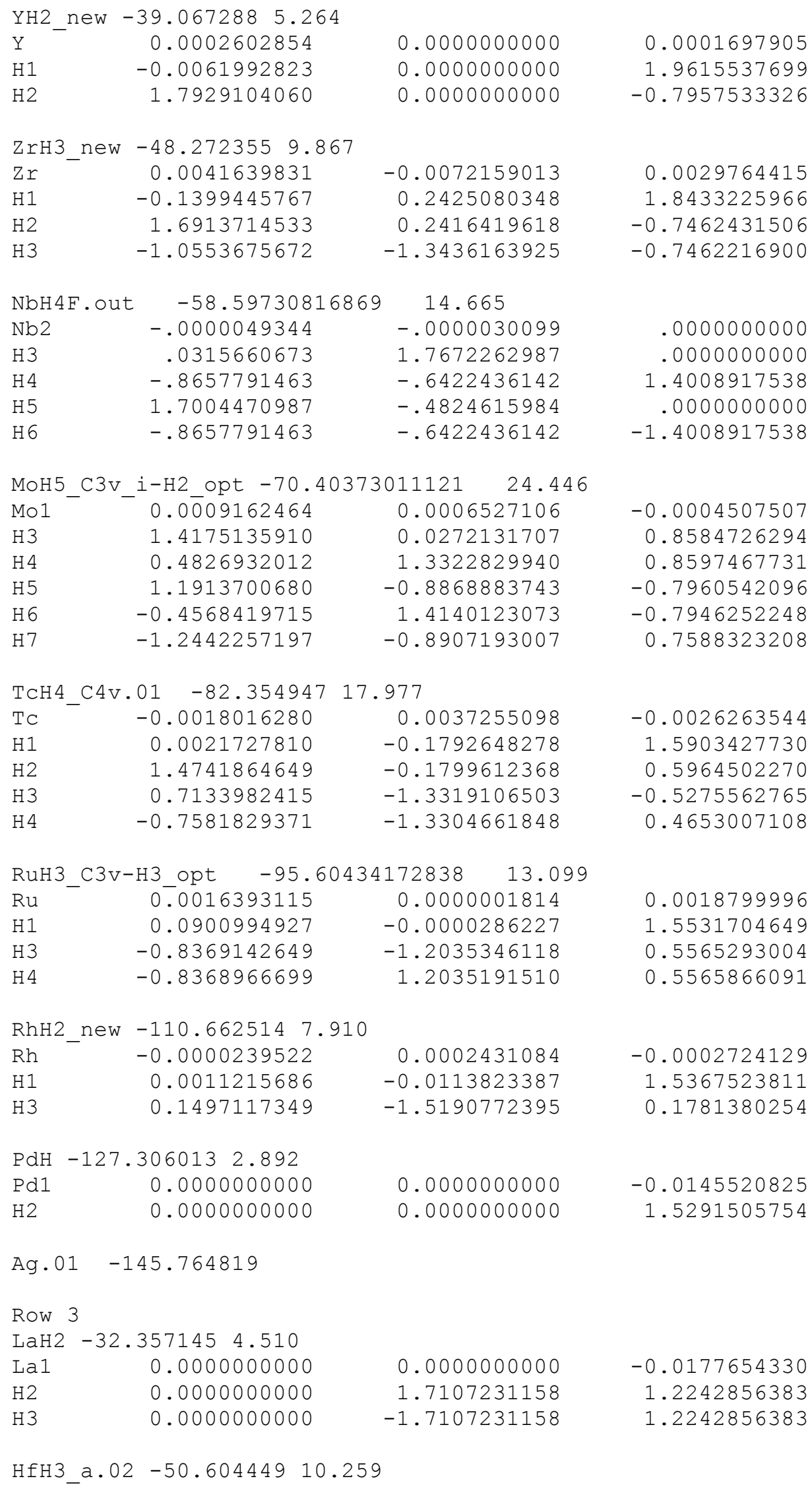




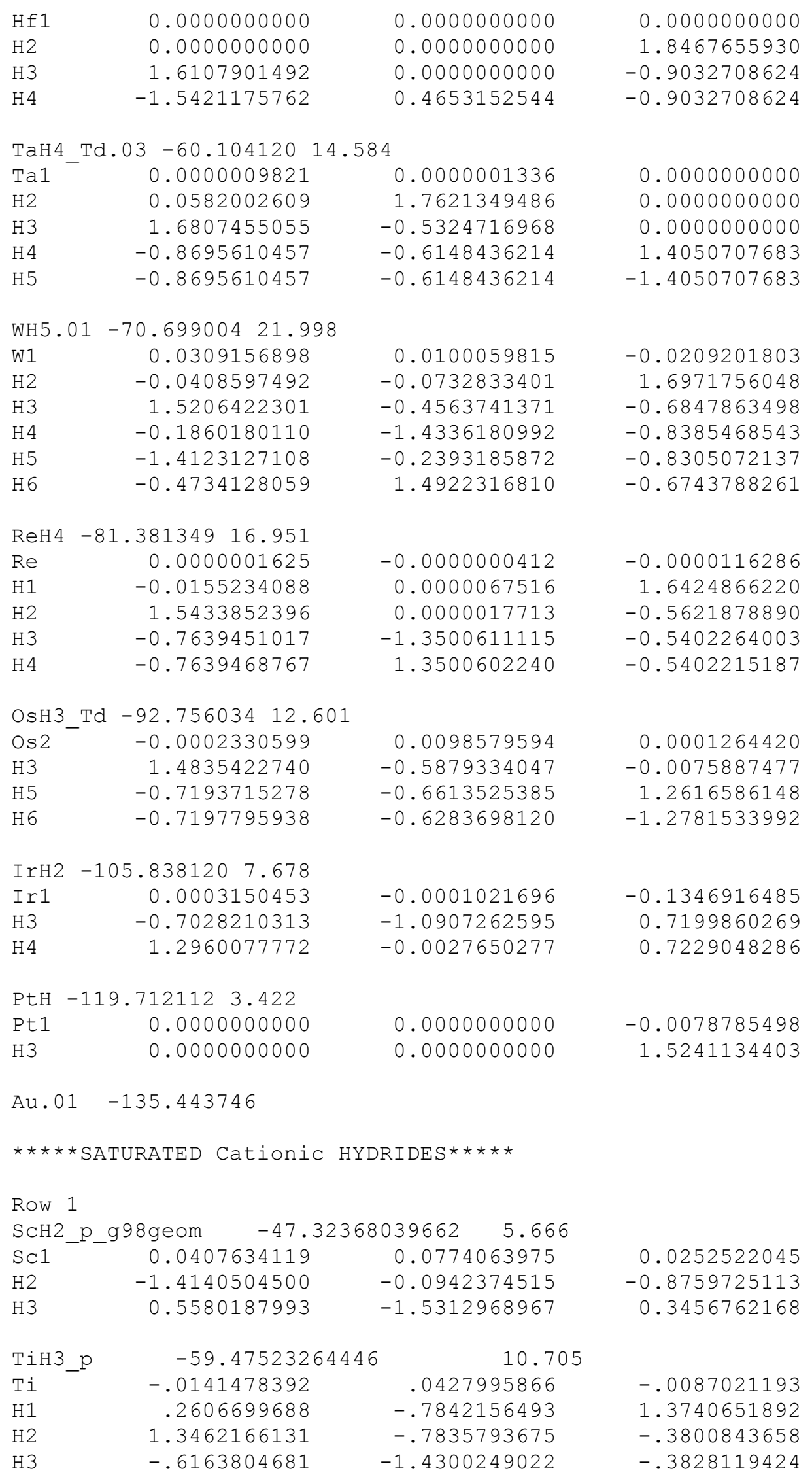




\begin{tabular}{|c|c|c|c|}
\hline VH $4 \mathrm{C} 4 \mathrm{~V}$ & -73.273946 & 18.673 & \\
\hline V1 & .0000000000 & .0000000000 & .0868103471 \\
\hline $\mathrm{H} 2$ & .7378907758 & .7378907758 & -1.0970314863 \\
\hline H3 & -.7378907758 & .7378907758 & -1.0970314863 \\
\hline H4 & -.7378907758 & -.7378907758 & -1.0970314863 \\
\hline H5 & .7378907758 & -.7378907758 & -1.0970314863 \\
\hline CrH5C4vUm & -88.802022 & 28.084 & \\
\hline $\operatorname{Cr} 1$ & .0000000000 & .0000000000 & -.0922884156 \\
\hline H2 & .8641385392 & .8641385392 & .8411571946 \\
\hline H3 & -.8641385392 & .8641385392 & .8411571946 \\
\hline H4 & -.8641385392 & -.8641385392 & .8411571946 \\
\hline H5 & .8641385392 & -.8641385392 & .8411571946 \\
\hline H 6 & .0000000000 & .0000000000 & 1.3916606544 \\
\hline MnH 6C5vu & -107.015187 & 36.507 & \\
\hline $\mathrm{H} 1$ & 0.0000000000 & 0.0000000000 & 1.3703249490 \\
\hline Mn2 & 0.0000000000 & 0.0000000000 & -.0868109026 \\
\hline H3 & 1.0246056483 & .7444195780 & .6723734348 \\
\hline H4 & 1.0246056483 & -.7444195780 & .6723734348 \\
\hline H5 & -.3913645326 & 1.2044961790 & .6723734348 \\
\hline H 6 & -1.2664822315 & 0.0000000000 & .6723734348 \\
\hline H7 & -.3913645326 & -1.2044961790 & .6723734348 \\
\hline FeH5C5V & -125.996233 & 28.62 & \\
\hline Fe1 & 0.0000000000 & 0.0000000000 & -.0764713909 \\
\hline H2 & 1.1241954296 & 0.0000000000 & .8488423465 \\
\hline H3 & .3473954927 & 1.0691733889 & .8488423465 \\
\hline H4 & -.9094932076 & .6607854942 & .8488423465 \\
\hline H5 & -.9094932076 & -.6607854942 & .8488423465 \\
\hline H 6 & .3473954927 & -1.0691733889 & .8488423465 \\
\hline $\mathrm{COH} 4 \mathrm{C} 4 \mathrm{~V}$ & -147.050416 & 21.948 & \\
\hline $\mathrm{Col}$ & .0000000000 & .0000000000 & -.0635304560 \\
\hline H2 & .0000000000 & 1.0073058337 & .9287458052 \\
\hline H3 & 1.0073058337 & .0000000000 & .9287458052 \\
\hline H4 & .0000000000 & -1.0073058337 & .9287458052 \\
\hline H5 & -1.0073058337 & .0000000000 & .9287458052 \\
\hline NiH3C3v & -170.713511 & 14.473 & \\
\hline $\mathrm{Ni}$ & .0013806153 & -.0012479279 & .0018464204 \\
\hline H1 & .1205540305 & -.0983649972 & 1.3940394092 \\
\hline $\mathrm{H} 2$ & 1.3410082956 & -.1028642880 & .4005681404 \\
\hline H3 & .3266758610 & -1.3059988555 & .3938679253 \\
\hline $\mathrm{CuH} 278$ & -196.970671 & 7.797 & \\
\hline $\mathrm{H} 1$ & .0000000000 & .9065460385 & 1.0847464392 \\
\hline $\mathrm{Cu} 2$ & .0000000000 & .0000000000 & -.0347446860 \\
\hline H3 & .0000000000 & -.9065460385 & 1.0847464392 \\
\hline \multicolumn{4}{|l|}{ Row 2} \\
\hline YH2_p -38 & .83547469 & & \\
\hline $\mathrm{Y}-$ & -0.0014749117 & 0.0000000000 & -0.0009619942 \\
\hline $\mathrm{H} 1$ & 0.1569165769 & 0.0000000000 & 1.8788180371 \\
\hline $\mathrm{H} 2$ & 1.7828659258 & 0.0000000000 & -0.6131765773 \\
\hline ZrH3_p & -47.99778 & 10.397 & \\
\hline
\end{tabular}




\begin{tabular}{|c|c|c|c|}
\hline Zr & -.0032145153 & .0073006931 & -.0020888677 \\
\hline $\mathrm{H} 1$ & .1249436036 & -.2837669436 & 1.7635446519 \\
\hline $\mathrm{H} 2$ & 1.6643585633 & -.2835364759 & -.5964009220 \\
\hline H3 & -.9140464613 & -1.4195009752 & -.5963953211 \\
\hline $\mathrm{NbH} 4 \mathrm{C} 3 \mathrm{viU} 2$ & -58.291915 & 17.321 & \\
\hline $\mathrm{H} 1$ & 0.0000000000 & 0.0000000000 & -1.7283687733 \\
\hline $\mathrm{Nb} 2$ & 0.0000000000 & 0.0000000000 & -.0000316072 \\
\hline H3 & 1.6293186129 & .0000000000 & .5770941602 \\
\hline H 4 & -.8146593064 & 1.4110313096 & .5770941602 \\
\hline H5 & -.8146593064 & -1.4110313096 & .5770941602 \\
\hline MoH5C 4 vUm & -70.086409 & 27.591 & \\
\hline Mo1 & .0000000000 & .0000000000 & -.0520760048 \\
\hline $\mathrm{H} 2$ & .9685570557 & .9685570557 & .8729704720 \\
\hline H3 & -.9685570557 & .9685570557 & .8729704720 \\
\hline H4 & -.9685570557 & -.9685570557 & .8729704720 \\
\hline H5 & .9685570557 & -.9685570557 & .8729704720 \\
\hline H6 & .0000000000 & .0000000000 & 1.5508110137 \\
\hline TcH6C5vu & -83.272042 & 34.763 & \\
\hline $\mathrm{H} 1$ & 0.0000000000 & 0.0000000000 & 1.5244832296 \\
\hline Tc2 & 0.0000000000 & 0.0000000000 & -.0541193984 \\
\hline H3 & 1.1199953522 & .8137242546 & .7234572830 \\
\hline H4 & 1.1199953522 & -.8137242546 & .7234572830 \\
\hline H5 & -.4278001573 & 1.3166335014 & .7234572830 \\
\hline H6 & -1.3843903898 & 0.0000000000 & .7234572830 \\
\hline H7 & -.4278001573 & -1.3166335014 & .7234572830 \\
\hline RuH5C5V & -96.519931 & 26.511 & \\
\hline Ru1 & .0000001763 & -.0449048313 & 0.0000000000 \\
\hline $\mathrm{H} 2$ & -1.2282550243 & .9181307735 & 0.0000000000 \\
\hline H3 & -.3897501450 & .9002869684 & 1.1775650531 \\
\hline H4 & 1.0038687432 & .9108818029 & .7155475646 \\
\hline H5 & 1.0038687432 & .9108818029 & -.7155475646 \\
\hline H6 & -.3897501450 & .9002869684 & -1.1775650531 \\
\hline $\mathrm{RhH} 4 \mathrm{C} 4 \mathrm{~V}$ & -111.557916 & 20.883 & \\
\hline Rh1 & .0000000000 & .0000000000 & -.0382650261 \\
\hline $\mathrm{H} 2$ & .0000000000 & 1.1331382667 & .9767771383 \\
\hline H3 & 1.1331382667 & .0000000000 & .9767771383 \\
\hline H4 & .0000000000 & -1.1331382667 & .9767771383 \\
\hline H5 & -1.1331382667 & .0000000000 & .9767771383 \\
\hline $\mathrm{PdH} 3 \mathrm{C} 3 \mathrm{v}$ & -128.182824 & 14.15 & \\
\hline $\mathrm{Pd}$ & -.0004906492 & .0001671838 & -.0008468422 \\
\hline H1 & .0680728170 & -.0603199269 & 1.5085170889 \\
\hline H2 & 1.4634602301 & -.0596362753 & .3736498811 \\
\hline H3 & .3021379599 & -1.4333467196 & .3710739550 \\
\hline AgH2 78 & -146.580747 & 7.28 & \\
\hline $\mathrm{H} 1$ & .0000000000 & .9836651697 & 1.1922460292 \\
\hline $\operatorname{Ag} 2$ & .0000000000 & .0000000000 & -.0224792918 \\
\hline H3 & .0000000000 & -.9836651697 & 1.1922460292 \\
\hline \multicolumn{4}{|l|}{ Row 3} \\
\hline \multicolumn{4}{|c|}{-32.14203273} \\
\hline
\end{tabular}




\begin{tabular}{|c|c|c|c|}
\hline La1 & -0.0000709285 & 0.0000000000 & -0.0000513594 \\
\hline $\mathrm{H} 2$ & 0.0629104763 & 0.0000000000 & 2.0377063621 \\
\hline H3 & 1.9570278537 & 0.0000000000 & -0.5711720377 \\
\hline HfH3_p & -50.335643 & 11.308 & \\
\hline $\mathrm{Hfl}$ & -.0000023195 & -.0000000144 & .0172695105 \\
\hline $\mathrm{H} 2$ & -1.5716033637 & .0000016110 & -.8054629165 \\
\hline H3 & .7860094055 & -1.3611898524 & -.8050470138 \\
\hline H4 & .7860081022 & 1.3611908155 & -.8050474668 \\
\hline TaH4C3viu & -59.817509 & 17.656 & \\
\hline $\mathrm{H} 1$ & 0.0000000000 & 0.0000000000 & 1.7233592751 \\
\hline Ta2 & 0.0000000000 & 0.0000000000 & .0004564795 \\
\hline H3 & -.8090955158 & 1.4013945416 & -.6017723333 \\
\hline $\mathrm{H} 4$ & 1.6181910317 & .0000000000 & -.6017723333 \\
\hline H5 & -.8090955158 & -1.4013945416 & -.6017723333 \\
\hline WH5CXX & -70.37973 & 26.691 & \\
\hline $\mathrm{H} 1$ & -.6342406469 & 1.5733176810 & .0000000000 \\
\hline W2 & .0051325628 & .0074912798 & .0000000000 \\
\hline H3 & -.9910846614 & -1.3046361698 & .0000000000 \\
\hline H4 & -.4983940990 & -.7178661487 & -1.4144196900 \\
\hline H5 & -.4983940990 & -.7178661487 & 1.4144196900 \\
\hline H6 & 1.6853043508 & -.2002777373 & .0000000000 \\
\hline ReH $6 \mathrm{p}$ & -82.301954 & 34.652 & \\
\hline $\operatorname{Re} 1^{-+}$ & -.0017134087 & -.0012228126 & -.0027592792 \\
\hline H2 & .0281229567 & .0200030700 & 1.6036398492 \\
\hline H3 & 1.4177983057 & .0199804655 & .7499621461 \\
\hline H4 & .4613661434 & 1.3406694475 & .7502497265 \\
\hline H5 & 1.2472071262 & -.8283087301 & -.6503236978 \\
\hline H6 & -.3982575306 & 1.4435023811 & -.6497422682 \\
\hline H7 & -1.1436212508 & -.8285130826 & .8188205094 \\
\hline OsH5C5V & -93.683161 & 27.943 & \\
\hline Os1 & -.0012738717 & -.0222758415 & .0000000000 \\
\hline $\mathrm{H} 2$ & -1.3191202181 & .8537128451 & .0000000000 \\
\hline H3 & -.4052381537 & .8583405621 & 1.2508462193 \\
\hline H4 & 1.0630831307 & .8562987052 & .7724310363 \\
\hline H5 & 1.0630831307 & .8562987052 & -.7724310363 \\
\hline H6 & -.4052381537 & .8583405621 & -1.2508462193 \\
\hline $\operatorname{IrH} 4 \mathrm{C} 4 \mathrm{~V}$ & -106.760941 & 22.064 & \\
\hline $\operatorname{Ir} 1$ & .0000000000 & .0000000000 & -.0197397669 \\
\hline $\mathrm{H} 2$ & .0000000000 & 1.2040274259 & .9448671677 \\
\hline H3 & 1.2040274259 & .0000000000 & .9448671677 \\
\hline H 4 & .0000000000 & -1.2040274259 & .9448671677 \\
\hline H5 & -1.2040274259 & .0000000000 & .9448671677 \\
\hline PtH3C3v & -120.635121 & 14.641 & \\
\hline Pt & .0023092490 & -.0014264443 & .0020378463 \\
\hline $\mathrm{H} 1$ & -.0642575079 & .0428343701 & 1.5259741710 \\
\hline H2 & 1.5053479164 & .0482689384 & .2496043077 \\
\hline H3 & .1997094447 & -1.4924007408 & .2531872089 \\
\hline $\mathrm{AuH} 2 \_\mathrm{p}$ & -136.305229 & 8.103 & \\
\hline $\mathrm{Aul}$ & .0000000000 & .0000000005 & -.0123619346 \\
\hline
\end{tabular}




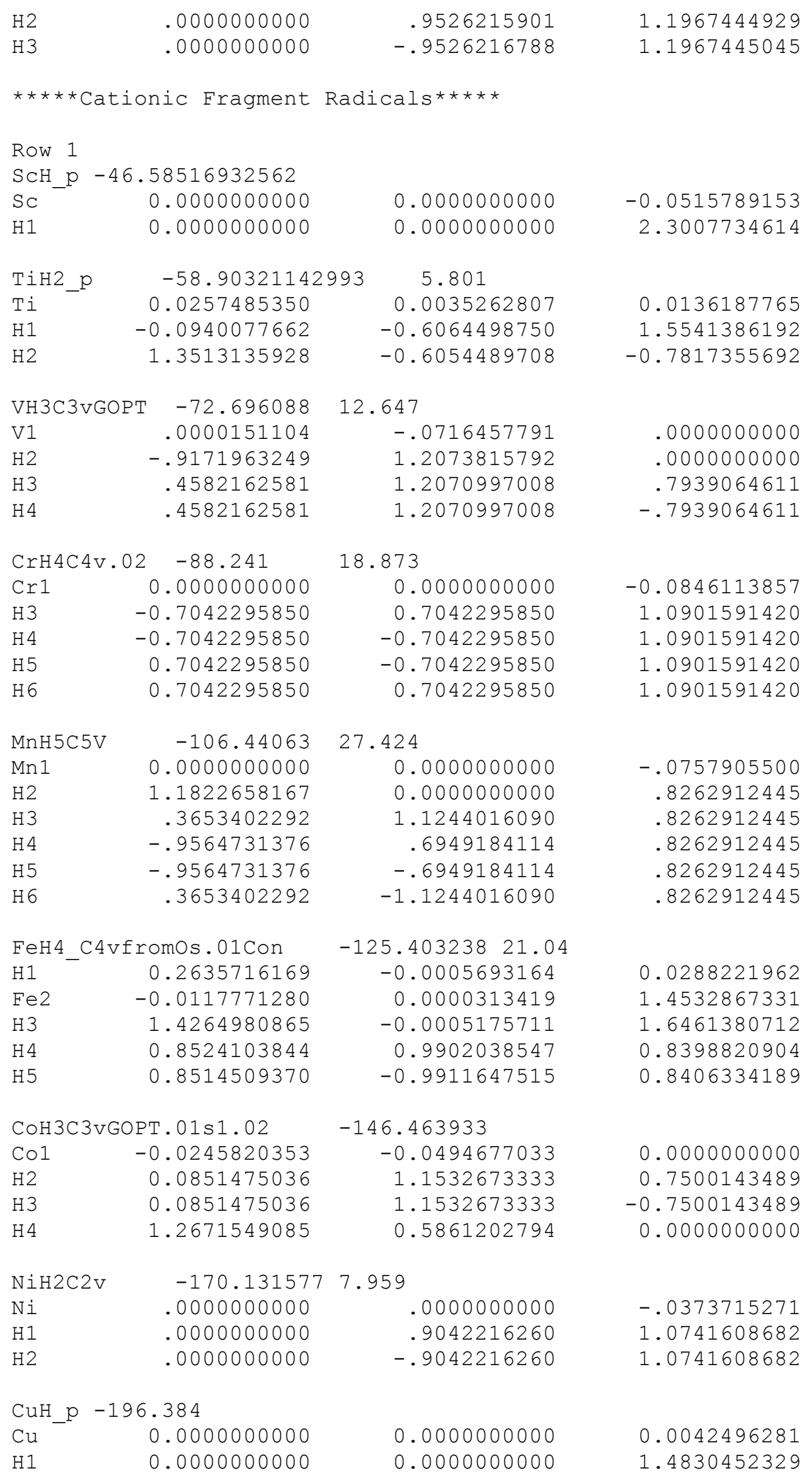




\begin{tabular}{|c|c|c|c|}
\hline \multicolumn{4}{|c|}{ YH_P $\quad-38.231$} \\
\hline $\mathrm{Y}^{-}$ & -0.0014603372 & 0.0000000000 & -0.0007889651 \\
\hline H1 & 0.1556308768 & 0.0000000000 & 1.8635541766 \\
\hline ZrH2_p.01 & -47.393 & 5.783 & \\
\hline Zr $-r$ & -0.0017778388 & 0.0068351413 & -0.0011515676 \\
\hline H1 & 0.0218591613 & -0.2630098074 & 1.7815479209 \\
\hline H2 & 1.6392818896 & -0.2627632990 & -0.6980176016 \\
\hline NbH3_p & -57.690 & 10.849 & \\
\hline $\mathrm{Nb}$ & -0.0179921966 & -0.0226535292 & 0.0000000000 \\
\hline H1 & 0.0589273153 & 1.4256876638 & 0.9160672993 \\
\hline H2 & 0.0589273153 & 1.4256876638 & -0.9160672993 \\
\hline H3 & 1.5407564966 & -0.7630591349 & 0.0000000000 \\
\hline $\mathrm{MoH} 4 \mathrm{C} 3 \mathrm{v}$ & C4v actually) & $-69.487364 \quad 14.964$ & \\
\hline $\mathrm{H} 1$ & -.7648862476 & 1.4272632117 & .0000000000 \\
\hline Mo2 & -.0178801162 & -.0433890340 & .0000000000 \\
\hline H3 & 1.5066164747 & .5866163514 & .0000000000 \\
\hline H4 & .3718288854 & 1.0026732567 & 1.2138112839 \\
\hline H5 & .3718288854 & 1.0026732567 & -1.2138112839 \\
\hline $\mathrm{TCH} 5 \mathrm{C} 5 \mathrm{~V}$ & -82.663823 & 26.744 & \\
\hline Tc1 & 0.0000000000 & 0.0000000000 & -.0433694545 \\
\hline H2 & 1.3284291779 & 0.0000000000 & .8426428005 \\
\hline H3 & .4105071918 & 1.2634112261 & .8426428005 \\
\hline H4 & -1.0747217807 & .7808310795 & .8426428005 \\
\hline H5 & -1.0747217807 & -.7808310795 & .8426428005 \\
\hline H6 & .4105071918 & -1.2634112261 & .8426428005 \\
\hline RuH 4C 4v1.0 & $01-95.89961429$ & 20.105 & \\
\hline H1 & 1.1258542624 & 0.0000000000 & 1.0429093728 \\
\hline Ru2 & 0.0000000000 & 0.0000000000 & -0.0412571270 \\
\hline H3 & -1.1258542624 & 0.0000000000 & 1.0429093728 \\
\hline H4 & 0.0000000000 & 1.1258542624 & 1.0429093728 \\
\hline H5 & 0.0000000000 & -1.1258542624 & 1.0429093728 \\
\hline $\mathrm{RhH} 3 \mathrm{C} 3 \mathrm{v}$ & -110.926071 & 12.551 & \\
\hline Rh2 & .0000000000 & .0000000000 & -.0345666650 \\
\hline H3 & .9490408893 & .0000000000 & 1.1764939258 \\
\hline H 4 & -.4745204447 & .8218935194 & 1.1764939258 \\
\hline H5 & -.4745204447 & -.8218935194 & 1.1764939258 \\
\hline $\mathrm{PdH} 2 \mathrm{C} 2 \mathrm{vCon}$ & n $\quad-127.56409$ & 7.978 & \\
\hline $\mathrm{Pd}$ & .0000000000 & .0000000000 & -.0220376031 \\
\hline H1 & .0000000000 & .9598435105 & 1.1578690985 \\
\hline $\mathrm{H} 2$ & .0000000000 & -.9598435105 & 1.1578690985 \\
\hline AgH_p -146 & 6.000 & & \\
\hline $\mathrm{Ag}-{ }^{-}$ & 0.0000000000 & 0.0000000000 & 0.0036833139 \\
\hline H1 & 0.0000000000 & 0.0000000000 & 1.7130136409 \\
\hline LaH_p -31 . & .528 & & \\
\hline La $1^{--}$ & 0.0000000000 & 0.0000000000 & 0.0000127148 \\
\hline & 0.0000000000 & 0.0000000000 & 2.0369783245 \\
\hline
\end{tabular}




\begin{tabular}{|c|c|c|c|}
\hline $\mathrm{HfH} 2 \mathrm{p}$ & -49.721 & 6.034 & \\
\hline $\mathrm{Hfl}$ & -0.0000574944 & -0.0000956208 & 0.0171539691 \\
\hline H2 & -1.5771741730 & 0.0145967735 & -0.7951499978 \\
\hline H3 & 0.8014316567 & -1.3587145550 & -0.7947300868 \\
\hline $\mathrm{TaH} 3 \mathrm{C} 3 \mathrm{v} \cdot 0$ & $1 \quad-59.19870933$ & 12.537 & \\
\hline Ta1 & -0.0142552995 & 0.0013161207 & 0.0000000000 \\
\hline $\mathrm{H} 2$ & 0.8240123531 & 1.5034575941 & 0.0000000000 \\
\hline 3 & 0.8677139095 & -0.8698789662 & 1.1940994637 \\
\hline 4 & 0.8677139095 & -0.8698789662 & -1.1940994637 \\
\hline WH4C3viU & -69.776947 & 18.461 & \\
\hline $\mathrm{H} 1$ & .0201814427 & 1.6775409409 & .0000000000 \\
\hline $\mathrm{H} 2$ & 1.6001875119 & -.5072120559 & .0000000000 \\
\hline H3 & -.8129836731 & -.5881115211 & -1.3456580779 \\
\hline W4 & .0000306723 & .0000322927 & .0000000000 \\
\hline H5 & -.8129836731 & -.5881115211 & 1.3456580779 \\
\hline eH5C5V & -81.687731 & 27.52 & \\
\hline e1 & 0.0000000000 & 0.0000000000 & -.0211904145 \\
\hline H2 & 1.3947013370 & .0000000000 & .7861820696 \\
\hline H3 & .4309864152 & 1.3264397949 & .7861820696 \\
\hline H4 & -1.1283370837 & .8197848773 & .7861820696 \\
\hline H5 & -1.1283370837 & -.8197848773 & .7861820696 \\
\hline H6 & .4309864152 & -1.3264397949 & .7861820696 \\
\hline $\mathrm{OsH} 4 \mathrm{C} 4 \mathrm{v}_{-} \mathrm{j}$ & amalguess. 01 & -93.05625402 & \\
\hline $\mathrm{H} 1$ & 1.2039933468 & 0.0000000000 & 1.0041657658 \\
\hline Os 2 & 0.0000000000 & 0.0000000000 & -0.0210884180 \\
\hline H3 & -1.2039933468 & 0.0000000000 & 1.0041657658 \\
\hline H4 & 0.0000000000 & 1.2038856883 & 1.0042006529 \\
\hline H5 & 0.0000000000 & -1.2038856883 & 1.0042006529 \\
\hline IrH3_p & -106.126399 & & \\
\hline $\mathrm{H} 1$ & -0.1985960152 & -0.0000165640 & 0.0600653116 \\
\hline $\operatorname{Ir} 2$ & 0.0026973550 & 0.0000000095 & 1.6024447415 \\
\hline H5 & -0.8920366111 & 1.2012819499 & 2.0217792558 \\
\hline H6 & -0.8920362779 & -1.2012730614 & 2.0218054034 \\
\hline PtH2C2v & -119.997904 & 8.536 & \\
\hline Pt & .0000000000 & .0000000000 & -.0118374736 \\
\hline H1 & .0000000000 & 1.0029347390 & 1.1449856327 \\
\hline $\mathrm{H} 2$ & .0000000000 & -1.0029347390 & 1.1449856327 \\
\hline $\mathrm{AuH}$ _p -13 & 35.680939 & & \\
\hline & 0.0000000000 & -0.0000126534 & -0.0123779964 \\
\hline $\mathrm{H} 2$ & 0.0000000000 & 0.9550946338 & 1.1998835758 \\
\hline
\end{tabular}

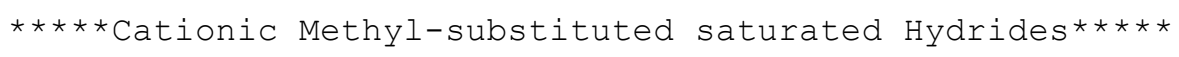

$\begin{array}{lrrr}\text { TiH2Me } & -58.903 & 32.315 & \\ \text { Ti1 } & -.0991979825 & -.4519490295 & .0000000000 \\ \text { C2 } & .1869085396 & 1.4972061173 & .0000000000\end{array}$




\begin{tabular}{|c|c|c|c|}
\hline H3 & .7574367757 & 1.7510120205 & .9044963352 \\
\hline $\mathrm{H} 4$ & .7574367757 & 1.7510120205 & -.9044963352 \\
\hline H5 & -.7985802108 & 1.9882176807 & .0000000000 \\
\hline 16 & .8888143875 & -.9077212069 & 1.2505540919 \\
\hline & .8888143875 & -.9077212069 & -1.2505540919 \\
\hline VH3MeC 3vL & -72.696088 & 39.603 & \\
\hline $\mathrm{H} 1$ & 1.0604956575 & .0000000000 & 1.7870750555 \\
\hline 2 & 0.0000000000 & 0.0000000000 & 1.4893228840 \\
\hline V3 & 0.0000000000 & 0.0000000000 & -.4033691565 \\
\hline H4 & -.5302478287 & .9184161800 & 1.7870750555 \\
\hline H5 & -.5302478287 & -.9184161800 & 1.7870750555 \\
\hline H6 & 1.5200169044 & .0000000000 & -.9015545993 \\
\hline H7 & -.7600084522 & 1.3163732534 & -.9015545993 \\
\hline $\mathrm{H} 8$ & -.7600084522 & -1.3163732534 & -.9015545993 \\
\hline $\mathrm{MnH} 5 \mathrm{MeCsC}$ & $5 \mathrm{ViUmCon}-106.4$ & $4063 \quad 57.155$ & \\
\hline h1 & 1.4407013370 & 1.4637672562 & .0000000000 \\
\hline$c 2$ & .3396588084 & 1.4720732218 & .0000000000 \\
\hline h3 & -.1288469412 & 1.8296091837 & .9299684502 \\
\hline h4 & -.1288469412 & 1.8296091837 & -.9299684502 \\
\hline mn 5 & -.0728472409 & -.3155582077 & .0000000000 \\
\hline h 6 & -1.4778362747 & -.7942298322 & .0000000000 \\
\hline h 7 & -.6289414196 & -1.0141053918 & 1.1842578433 \\
\hline h8 & -.6289414196 & -1.0141053918 & -1.1842578433 \\
\hline h 9 & .7397322278 & -1.3133594367 & .7382581422 \\
\hline h10 & .7397322278 & -1.3133594367 & -.7382581422 \\
\hline $\mathrm{CoH} 3 \mathrm{Me}$ & -146.463933 & 42.647 & \\
\hline Col & .0019546676 & .0002445615 & .0767001287 \\
\hline C2 & -.0081783466 & -.0007558009 & 1.8950247192 \\
\hline H3 & 1.3123827735 & -.0011165735 & -.4876978376 \\
\hline H4 & -.6481970312 & 1.1315123757 & -.4996468967 \\
\hline H5 & -.6485585988 & -1.1322631693 & -.4976602373 \\
\hline H6 & -1.0811493765 & -.0009819406 & 2.1280109685 \\
\hline $\mathrm{H} 7$ & .5258258560 & -.9287670760 & 2.1385114878 \\
\hline H8 & .5258926086 & .9269110733 & 2.1390922570 \\
\hline $\mathrm{NiH} 2 \mathrm{MeCsC}$ & on -170.131577 & 36.62 & \\
\hline $\mathrm{H} 1$ & 1.1814777303 & 1.4845523646 & .0000000000 \\
\hline C2 & .0797070222 & 1.4678362582 & .0000000000 \\
\hline $\mathrm{Ni3}$ & .0147214127 & -.4044919259 & .0000000000 \\
\hline H4 & -.3711611392 & 1.8605430031 & -.9103712726 \\
\hline H5 & -.3711611392 & 1.8605430031 & .9103712726 \\
\hline H 6 & -1.0631484071 & -.2885292621 & -.8848238683 \\
\hline H7 & -1.0631484071 & -.2885292621 & .8848238683 \\
\hline CuHMeCon & -196.384 & 28.135 & \\
\hline $\mathrm{C} 1$ & .0617120872 & 1.5497423607 & .0000000000 \\
\hline $\mathrm{Cu} 2$ & -.0349924661 & -.3734049153 & .0000000000 \\
\hline H3 & -1.0395422989 & 1.5661933239 & .0000000000 \\
\hline $\mathrm{H} 4$ & .5373657043 & 1.8716908297 & .9218014911 \\
\hline H5 & .5373657043 & 1.8716908297 & -.9218014911 \\
\hline H 6 & 1.4149801586 & -.4463161048 & .0000000000 \\
\hline $\mathrm{ZrH} 2 \mathrm{Me}$ & -47.393 & 31.369 & \\
\hline Zr1 & .0568967020 & .0019657391 & .0364952534 \\
\hline
\end{tabular}




\begin{tabular}{|c|c|c|c|}
\hline $\mathrm{C} 2$ & -.4223915208 & -.0137398070 & 2.1045245495 \\
\hline H3 & .4415923056 & -.0151919204 & 2.7837726149 \\
\hline H4 & -1.0422158577 & .8755859778 & 2.3028696315 \\
\hline H5 & -1.0277044696 & -.9174434806 & 2.2838319527 \\
\hline H 6 & -.8093650987 & -1.4710449271 & -.5429968175 \\
\hline H7 & -.9286466230 & 1.3954773913 & -.5498876966 \\
\hline $\mathrm{NbH} 3 \mathrm{Me}$ & -57.690 & 38.825 & \\
\hline $\mathrm{Nb} 1$ & .0013064734 & -.0017271702 & .0166792426 \\
\hline $\mathrm{C} 2$ & -.0041048252 & -.0004776759 & 2.0605615966 \\
\hline H3 & 1.6653794309 & .0001054990 & -.4769362557 \\
\hline H 4 & -.8321133216 & -1.4412554885 & -.4810749137 \\
\hline H5 & -.8301265281 & 1.4400296192 & -.4775550324 \\
\hline H 6 & -.5272948899 & -.9069106795 & 2.4090091543 \\
\hline $\mathrm{H} 7$ & 1.0422391642 & .0011461159 & 2.4100836049 \\
\hline H 8 & -.5286418104 & .9060054678 & 2.4067652320 \\
\hline $\mathrm{MoH} 4 \mathrm{Me}$ & -69.487364 & 45.186 & \\
\hline $\mathrm{H} 1$ & 1.5075102824 & -.7637252400 & -.0021294289 \\
\hline Mo2 & -.0862893982 & -.3779378455 & .0009280472 \\
\hline H3 & -1.2602716817 & -1.5904467498 & -.0012777421 \\
\hline $\mathrm{H} 4$ & .8409527740 & -.5841143731 & 1.3684772906 \\
\hline H5 & .8353625136 & -.5840030819 & -1.3706148108 \\
\hline C6 & -.5076727874 & 1.5891100692 & -.0012792742 \\
\hline H7 & -1.5856820480 & 1.7905226586 & -.0042082546 \\
\hline H 8 & .0002098692 & 1.9847676615 & .8967450910 \\
\hline H9 & .0050135716 & 1.9848661958 & -.8964489075 \\
\hline TcH5Me & -82.663823 & 54.365 & \\
\hline $\mathrm{C} 1$ & -.0000172224 & 1.7610287208 & 0.0000000000 \\
\hline TC2 & -.0000292202 & -.2276539902 & 0.0000000000 \\
\hline H3 & -1.4048630695 & -.9987634071 & 0.0000000000 \\
\hline H4 & -.4219407234 & -1.0140753234 & 1.3298858070 \\
\hline H5 & 1.1253804290 & -1.0032461878 & .8353385585 \\
\hline H 6 & -.4219407234 & -1.0140753234 & -1.3298858070 \\
\hline $\mathrm{H} 7$ & 1.1253804290 & -1.0032461878 & -.8353385585 \\
\hline H 8 & 1.0594304351 & 2.0578190720 & 0.0000000000 \\
\hline H9 & -.5292015266 & 2.0616153438 & -.9165895012 \\
\hline $\mathrm{H} 10$ & -.5292015266 & 2.0616153438 & .9165895012 \\
\hline RuH4MeCon & -95.89961429 & 47.389 & \\
\hline $\mathrm{C} 1$ & .0040966558 & 1.7489993506 & .0000000000 \\
\hline ru2 & .0143016835 & -.2285186222 & .0000000000 \\
\hline h3 & .4390755115 & -1.0215485755 & 1.2853876011 \\
\hline h 4 & .4390755115 & -1.0215485755 & -1.2853876011 \\
\hline h5 & -1.1903700341 & -.8867136362 & .7496810734 \\
\hline h 6 & -1.1903700341 & -.8867136362 & -.7496810734 \\
\hline h 7 & 1.0716435479 & 2.0178955306 & .0000000000 \\
\hline h 8 & -.5319603773 & 2.0399138881 & -.9126294801 \\
\hline h9 & -.5319603773 & 2.0399138881 & .9126294801 \\
\hline RhH3Me & -110.926071 & 40.823 & \\
\hline C1 & 0.0000000000 & 0.0000000000 & 1.7415751158 \\
\hline $\mathrm{H} 2$ & 1.0628962838 & .0000000000 & 2.0101510540 \\
\hline H3 & -.5314481419 & .9204951834 & 2.0101510540 \\
\hline H4 & -.5314481419 & -.9204951834 & 2.0101510540 \\
\hline Rh 5 & .0000000000 & .0000000000 & -.2367229625 \\
\hline
\end{tabular}




\begin{tabular}{|c|c|c|c|}
\hline H6 & -1.3991362461 & .0000000000 & -.8653774705 \\
\hline $\mathrm{H} 7$ & .6995681231 & -1.2116875325 & -.8653774705 \\
\hline H8 & .6995681231 & 1.2116875325 & -.8653774705 \\
\hline $\mathrm{PdH} 2 \mathrm{Me}$ & -127.56409 & 33.722 & \\
\hline $\mathrm{H} 1$ & 1.0026735003 & 1.6597398652 & .8047705342 \\
\hline C2 & .0421320442 & 1.6147516705 & .2859079145 \\
\hline $\mathrm{Pd} 3$ & -.1084282931 & -.3984694356 & -.0526346081 \\
\hline H4 & .0552260362 & 1.9921779178 & -.7393362832 \\
\hline H5 & -.7818679424 & 2.0045325586 & .8754783204 \\
\hline H 6 & -.6866751182 & -.4496780332 & 1.3443031068 \\
\hline H7 & -1.5942858019 & -.1310427292 & -.1506504491 \\
\hline AgHMeCon & -146.000 & 26.229 & \\
\hline $\mathrm{C} 1$ & -.1459196600 & 1.8704701946 & .0000000000 \\
\hline $\operatorname{Ag} 2$ & .0075561560 & -.2705853430 & .0000000000 \\
\hline H3 & 1.5264168109 & .1678132217 & .0000000000 \\
\hline H4 & .8149735600 & 2.3702345200 & .0000000000 \\
\hline H5 & -.7027348096 & 1.9464692544 & .9327496984 \\
\hline H 6 & -.7027348096 & 1.9464692544 & -.9327496984 \\
\hline $\mathrm{H} f \mathrm{H} 2 \mathrm{Me}$ & -49.721 & 32.447 & \\
\hline Hfl & .0646767317 & .0032045065 & .0156661675 \\
\hline C2 & -.4640745219 & -.0238595537 & 2.0477870059 \\
\hline H3 & .4017013975 & -.0613348557 & 2.7264773287 \\
\hline $\mathrm{H} 4$ & -1.0528630421 & .8791285283 & 2.2722771106 \\
\hline H5 & -1.0948384361 & -.9075061079 & 2.2321946581 \\
\hline H 6 & -.8294090126 & -1.4333720887 & -.5532846877 \\
\hline H7 & -1.0026737088 & 1.3127089204 & -.5593873631 \\
\hline TaH3Me & -59.19870933 & 38.966 & \\
\hline Tal & 0.0000000000 & 0.0000000000 & -.1505691902 \\
\hline C2 & 0.0000000000 & 0.0000000000 & 1.8753589967 \\
\hline H3 & 1.5677705119 & -.5333584848 & -.6674540693 \\
\hline H4 & -.3219832588 & 1.6244083330 & -.6674540693 \\
\hline H5 & -1.2457872531 & -1.0910498482 & -.6674540693 \\
\hline H6 & -.2023115183 & 1.0236580168 & 2.2354793283 \\
\hline $\mathrm{H} 7$ & -.7853580882 & -.6870359227 & 2.2354793283 \\
\hline H8 & .9876696065 & -.3366220941 & 2.2354793283 \\
\hline WH $4 \mathrm{Me}-69$ & $.776947 \quad 45.078$ & & \\
\hline W1 & -.0074278604 & .0078624811 & -.0124111011 \\
\hline $\mathrm{H} 2$ & .0130782671 & -.3264963477 & 1.6148710900 \\
\hline H3 & 1.4535355847 & -.3193473210 & .7076360349 \\
\hline $\mathrm{H} 4$ & -1.1447042882 & .8473915321 & .9000006885 \\
\hline H5 & 1.3024443077 & .8559821997 & -.6415407063 \\
\hline C 6 & -.4808253304 & -1.7446368276 & -.7782066381 \\
\hline H7 & -1.5123271278 & -2.1009773793 & -.6893271813 \\
\hline H 8 & .0528096870 & -2.0908234522 & -1.6695795761 \\
\hline H9 & .1013282435 & -2.1027908675 & .1467452383 \\
\hline $\mathrm{ReH} 5 \mathrm{Me}$ & -81.687731 & 55.162 & \\
\hline $\mathrm{H} 1$ & .4375758986 & -.8686776080 & 1.3772569776 \\
\hline Re2 & .0001801517 & -.1310087142 & 0.0000000000 \\
\hline H3 & -1.1696737432 & -.8549546847 & .8613219406 \\
\hline H4 & -1.1696737432 & -.8549546847 & -.8613219406 \\
\hline H5 & .4375758986 & -.8686776080 & -1.3772569776 \\
\hline
\end{tabular}




\begin{tabular}{|c|c|c|c|}
\hline H 6 & 1.4534401106 & -.8541870355 & 0.0000000000 \\
\hline $\mathrm{C} 7$ & -.0011895923 & 1.8526240995 & 0.0000000000 \\
\hline H8 & -1.0550921435 & 2.1766823594 & 0.0000000000 \\
\hline H9 & .5232965473 & 2.1842774502 & .9112095068 \\
\hline $\mathrm{H} 10$ & .5232965473 & 2.1842774502 & -.9112095068 \\
\hline $\mathrm{OsH} 4 \mathrm{Me}$ & -93.05838132 & 48.302 & \\
\hline Os 1 & .0064658252 & .0043150620 & -.0087645325 \\
\hline H2 & -.1429016148 & -.0044730297 & 1.5830143590 \\
\hline H3 & 1.5983848401 & -.0753263206 & -.1150454776 \\
\hline H4 & -.7948061365 & -1.3360254344 & .3221234143 \\
\hline H5 & .3135892403 & -1.3782356641 & -.7430805171 \\
\hline $\mathrm{C} 6$ & -.8619655771 & 1.4892662384 & -.9442233793 \\
\hline H7 & -.3704580809 & 2.3588269658 & -.4584260464 \\
\hline H8 & -.6258328607 & 1.4206876590 & -2.0146843852 \\
\hline H9 & -1.9417679064 & 1.4527416833 & -. 7420619163 \\
\hline $\mathrm{I} r \mathrm{H} 3 \mathrm{Me}$ & -106.126399 & 41.683 & \\
\hline C1 & 0.0000000000 & .0000000000 & 1.8301665797 \\
\hline H2 & 1.0574307683 & .0000000000 & 2.1304471437 \\
\hline H3 & -.5287153841 & .9157619081 & 2.1304471437 \\
\hline H4 & -.5287153841 & -.9157619081 & 2.1304471437 \\
\hline $\operatorname{Ir} 5$ & 0.0000000000 & .0000000000 & -.1352908567 \\
\hline H6 & -1.4299840255 & .0000000000 & -.7597990728 \\
\hline $\mathrm{H} 7$ & .7149920128 & -1.2384024931 & -.7597990728 \\
\hline H8 & .7149920128 & 1.2384024931 & -.7597990728 \\
\hline PtH2Me & -119.997904 & 35.199 & \\
\hline Pt1 & .0164199713 & .0269392496 & -.0005016754 \\
\hline C2 & -.1819769231 & -.2693974717 & 2.0168366081 \\
\hline H3 & .7054775696 & -.8444356136 & 2.2965892889 \\
\hline $\mathrm{H} 4$ & -.1910629658 & .7394592700 & 2.4396749444 \\
\hline H5 & -1.1031912573 & -.8100229526 & 2.2283408174 \\
\hline H 6 & -.4592023622 & -1.3986789736 & -.2546825657 \\
\hline H7 & -1.4695556547 & .3519999813 & -.0969443065 \\
\hline $\mathrm{AuHMe}$ & -135.68093928 .751 & & \\
\hline Aul 1 & .0723176856 & .0018932427 & .0006124133 \\
\hline C2 & -.8281511759 & -.0229279203 & 1.8964222990 \\
\hline H3 & .0619064762 & -.0171718396 & 2.5301995454 \\
\hline H4 & -1.4245089225 & .8836646806 & 1.9592790363 \\
\hline H5 & -1.4030415038 & -.9440779001 & 1.9461290335 \\
\hline H 6 & -1.3810291607 & -.0157987010 & -.5033008512 \\
\hline
\end{tabular}

\section{D мм $_{\text {parameters* used in solving Equation } 5}$}

\begin{tabular}{|c|c|c|c|c|c|c|c|c|c|c|}
\hline $\mathrm{Sc}$ & $\mathrm{Ti}$ & $\mathrm{V}$ & $\mathrm{Cr}$ & $\mathrm{Y}$ & $\mathrm{Zr}$ & $\mathrm{Nb}$ & $\mathrm{Mo}$ & $\mathrm{Hf}$ & $\mathrm{Ta}$ & $\mathrm{W}$ \\
\hline 35.67 & 27.43 & 15.74 & 21.61 & 36.86 & 44.58 & 29.52 & 38.44 & 53.41 & 36.97 & 42.25 \\
\hline
\end{tabular}

* Values were obtained by solving Equation 5 initially with computed $\mathrm{D}_{\mathrm{MH}}$ and $\mathrm{k}=8.4853786$, rather than from first principles. 
LACV3P++** basis from Schrödinger, Inc.

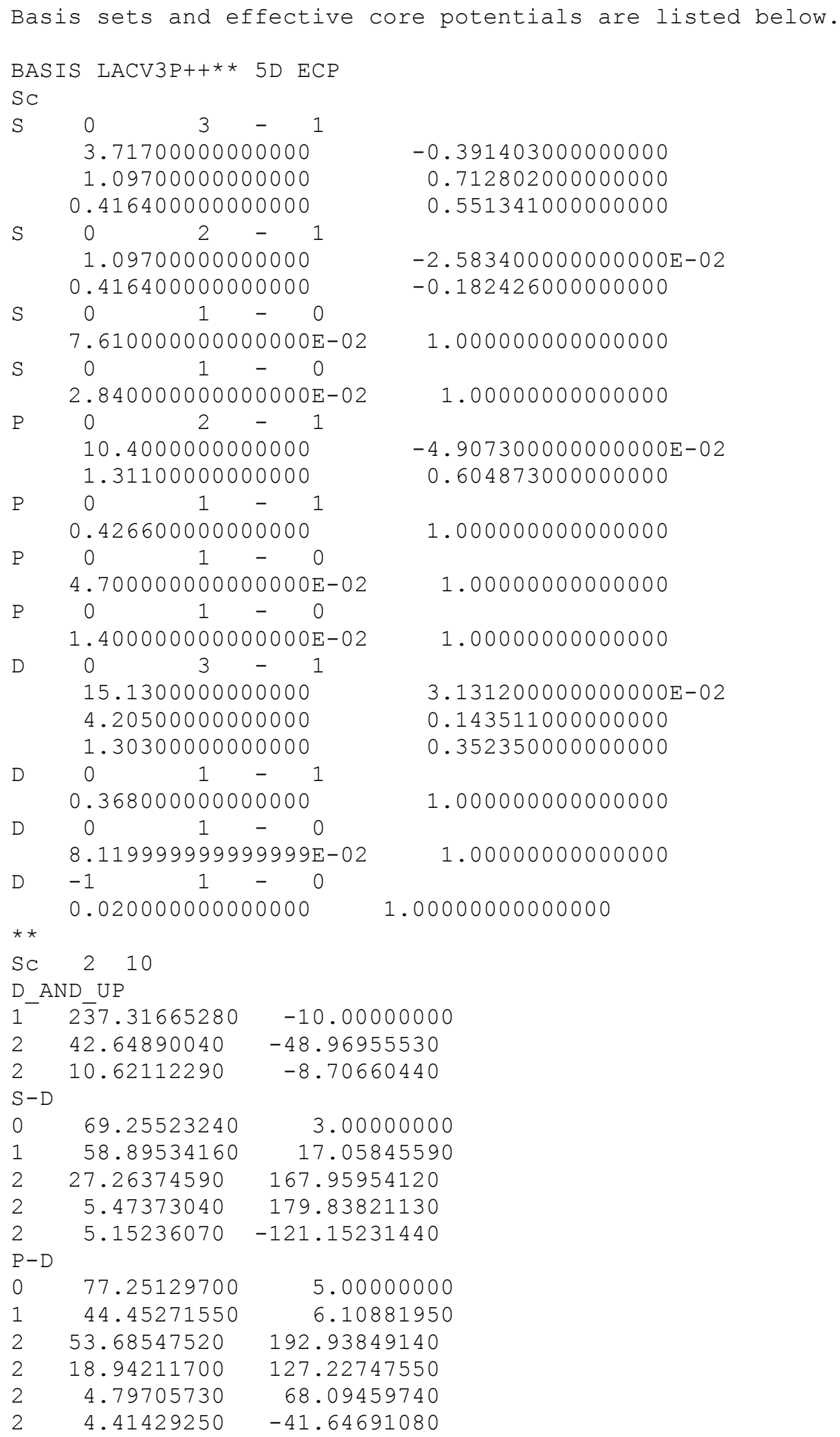


Ti

\begin{tabular}{|c|c|c|c|}
\hline & \multicolumn{2}{|c|}{$\begin{array}{llll}0 & 3 & - & 1\end{array}$} & \\
\hline & \multirow{2}{*}{\multicolumn{2}{|c|}{$\begin{array}{l}4.37200000000000 \\
1.09800000000000\end{array}$}} & -0.363439000000000 \\
\hline & & & 0.817934000000000 \\
\hline & \multicolumn{2}{|c|}{0.417800000000000} & 0.418470000000000 \\
\hline \multirow[t]{3}{*}{ S } & \multirow{2}{*}{\multicolumn{2}{|c|}{$\begin{array}{l}0 \\
1.09800000000000^{2}-{ }^{1}\end{array}$}} & \\
\hline & & & $-4.529800000000000 \mathrm{E}-02$ \\
\hline & \multicolumn{2}{|c|}{0.417800000000000} & -0.166081000000000 \\
\hline & $0 \quad 1$ & $-\quad 0$ & \\
\hline & \multicolumn{2}{|c|}{$8.720000000000000 \mathrm{E}-02$} & 1.000000000000000 \\
\hline & $0 \quad 1$ & -0 & \\
\hline & \multicolumn{2}{|c|}{$3.140000000000000 \mathrm{E}-02$} & 1.00000000000000 \\
\hline & $0 \quad 2$ & -1 & \\
\hline & \multicolumn{2}{|c|}{12.5200000000000} & $-4.552800000000000 \mathrm{E}-02$ \\
\hline & \multicolumn{2}{|c|}{1.49100000000000} & 0.618127000000000 \\
\hline & $0 \quad 1$ & -1 & \\
\hline & \multicolumn{2}{|c|}{0.485900000000000} & 1.000000000000000 \\
\hline & $0 \quad 1$ & -0 & \\
\hline & \multicolumn{2}{|c|}{$5.300000000000000 \mathrm{E}-02$} & 1.00000000000000 \\
\hline$P$ & $0 \quad 1$ & -0 & \\
\hline & \multicolumn{2}{|c|}{$1.600000000000000 \mathrm{E}-02$} & 1.00000000000000 \\
\hline \multirow[t]{4}{*}{ D } & \multirow{2}{*}{\multicolumn{2}{|c|}{$\frac{0}{20.2100000000000^{1}}$}} & \\
\hline & & & $2.872600000000000 \mathrm{E}-02$ \\
\hline & \multicolumn{2}{|c|}{5.49500000000000} & 0.143765000000000 \\
\hline & \multicolumn{2}{|c|}{1.69900000000000} & 0.370411000000000 \\
\hline D & $0 \quad 1$ & $\begin{array}{llll}- & 1 & r & r\end{array}$ & \\
\hline & 0.48400000000 & 00000 & 1.000000000000000 \\
\hline & $0 \quad 1$ & -0 & \\
\hline & 0.11570000000 & 00000 & 1.00000000000000 \\
\hline & $-1 \quad 1$ & -0 & \\
\hline & 0.02900000000 & 00000 & 1.00000000000000 \\
\hline & & & \\
\hline 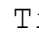 & 210 & & \\
\hline & ID_UP & & \\
\hline & $2 \overline{6} 5.32639090$ & -10.000 & 0000 \\
\hline 2 & 47.76878150 & -51.8427 & 160 \\
\hline 2 & 11.89033340 & -9.1429 & 450 \\
\hline & & & \\
\hline 0 & 81.47036960 & 3.000 & 0000 \\
\hline 1 & 72.64967240 & 19.482 & 5790 \\
\hline 2 & 31.81282130 & 207.3349 & \\
\hline 2 & 6.16644680 & 235.6744 & 010 \\
\hline 2 & 5.82683470 & -166.8784 & 870 \\
\hline & & & \\
\hline 0 & 50.29669430 & 5.000 & 0000 \\
\hline 1 & 63.50897540 & 5.534 & 8220 \\
\hline 2 & 26.09960840 & 177.8419 & \\
\hline 2 & 5.60225730 & 107.4207 & 530 \\
\hline 2 & 5.21710690 & -71.9065 & \\
\hline & & & \\
\hline & & & \\
\hline & $0 \quad 3$ & -1 & \\
\hline & 4.5900000000 & 00000 & -0.425305000000000 \\
\hline & 1.4930000000 & 00000 & 0.703564000000000 \\
\hline & 0.55700000000 & 00000 & 0.590172000000000 \\
\hline & $0 \quad 2$ & $\begin{array}{ll}- & 1\end{array}$ & \\
\hline & 1.4930000000 & 00000 & $-2.530400000000000 \mathrm{E}-02$ \\
\hline
\end{tabular}




\begin{tabular}{|c|c|c|c|}
\hline \multicolumn{3}{|c|}{0.557000000000000} & \multirow[t]{2}{*}{-0.165228000000000} \\
\hline \multirow[t]{3}{*}{$\mathrm{S}$} & $0 \quad 1$ & -0 & \\
\hline & 9.75000000000 & $00000 \mathrm{E}-02$ & 1.000000000000000 \\
\hline & $0 \quad 1$ & -0 & \\
\hline $\mathrm{S}$ & 3.42000000000 & $00000 \mathrm{E}-02$ & 1.00000000000000 \\
\hline \multirow[t]{3}{*}{$\mathrm{P}$} & $0 \quad 2$ & -1 & \\
\hline & 13.760000000 & 00000 & $-4.805500000000000 \mathrm{E}-02$ \\
\hline & 1.7120000000 & 00000 & 0.611873000000000 \\
\hline \multirow[t]{2}{*}{$\mathrm{P}$} & $0 \quad 1$ & -1 & \\
\hline & 0.55870000000 & 00000 & 1.000000000000000 \\
\hline \multirow[t]{2}{*}{$\mathrm{P}$} & $0 \quad 1$ & -0 & \\
\hline & 5.90000000000 & $00000 \mathrm{E}-02$ & 1.00000000000000 \\
\hline \multirow[t]{2}{*}{$\mathrm{P}$} & $0 \quad 1$ & $-\quad 0$ & \\
\hline & 1.80000000000 & $00000 \mathrm{E}-02$ & 1.00000000000000 \\
\hline \multirow[t]{4}{*}{ D } & $0 \quad 3$ & -1 & \\
\hline & 25.700000000 & 00000 & $2.783700000000000 \mathrm{E}-02$ \\
\hline & 6.5300000000 & 00000 & 0.151007000000000 \\
\hline & 2.0780000000 & 00000 & 0.367736000000000 \\
\hline \multirow[t]{2}{*}{ D } & $0 \quad 1$ & $-\quad 1$ & \\
\hline & 0.62430000000 & 00000 & 1.000000000000000 \\
\hline \multirow[t]{2}{*}{ D } & $0 \quad 1$ & -0 & \\
\hline & 0.15420000000 & 00000 & 1.00000000000000 \\
\hline \multirow[t]{2}{*}{ D } & $-1 \quad 1$ & -0 & \\
\hline & 0.03800000000 & 00000 & 1.00000000000000 \\
\hline \multicolumn{4}{|c|}{ 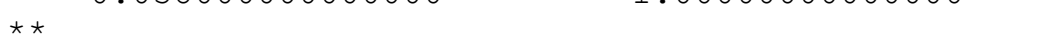 } \\
\hline $\mathrm{V}$ & 210 & & \\
\hline \\
\hline 1 & \multicolumn{3}{|c|}{ D_AND_UP } \\
\hline 2 & 53.50150520 & \multicolumn{2}{|c|}{$\begin{array}{r}-10.00000000 \\
-54.89098080\end{array}$} \\
\hline 2 & 13.32045100 & \multicolumn{2}{|c|}{-9.70105860} \\
\hline \multicolumn{4}{|c|}{$S-D$} \\
\hline 0 & 93.21936540 & \multicolumn{2}{|c|}{3.00000000} \\
\hline 1 & 71.10295670 & 22.534 & 6890 \\
\hline 2 & 33.87669930 & 216.5634 & 090 \\
\hline 2 & 6.86912000 & 252.6822 & 380 \\
\hline 2 & 6.48047220 & -176.2671 & 540 \\
\hline 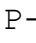 & & & \\
\hline 0 & 52.09539770 & 5.000 & 0000 \\
\hline 1 & 65.67784110 & 5.700 & 5550 \\
\hline 2 & 27.47037640 & 182.9054 & 740 \\
\hline 2 & 6.23520200 & 117.2756 & 260 \\
\hline 2 & 5.81399090 & -78.5829 & 340 \\
\hline & & & \\
\hline $\mathrm{Cr}$ & & & \\
\hline S & $0 \quad 3$ & -1 & \\
\hline & 5.3610000000 & 00000 & -0.379604000000000 \\
\hline & 1.4490000000 & 00000 & 0.777950000000000 \\
\hline & 0.54960000000 & 00000 & 0.473200000000000 \\
\hline S & $0 \quad 2$ & $-\quad 1$ & \\
\hline & 1.4490000000 & 00000 & $-4.090000000000000 \mathrm{E}-02$ \\
\hline & 0.54960000000 & 00000 & -0.148646000000000 \\
\hline S & $0 \quad 1$ & -0 & \\
\hline & 0.10520000000 & 00000 & 1.000000000000000 \\
\hline $\mathrm{S}$ & $0 \quad 1$ & $-\quad 0$ & \\
\hline & 3.64000000000 & $00000 \mathrm{E}-02$ & 1.00000000000000 \\
\hline P & $0 \quad 2$ & -1 & \\
\hline & 16.420000000 & 00000 & $-4.597800000000000 \mathrm{E}-02$ \\
\hline
\end{tabular}




\begin{tabular}{|c|c|c|c|}
\hline \multirow{3}{*}{$\mathrm{P}$} & \multicolumn{2}{|c|}{1.91400000000000} & 0.608849000000000 \\
\hline & $\begin{array}{ll}0 & 1\end{array}$ & $-\quad 1$ & \\
\hline & \multicolumn{2}{|c|}{0.624100000000000} & 1.000000000000000 \\
\hline \multirow[t]{2}{*}{$\mathrm{P}$} & $\begin{array}{ll}0 & 1\end{array}$ & $-\quad 0$ & \\
\hline & \multicolumn{2}{|c|}{$6.300000000000000 E-02$} & 1.00000000000000 \\
\hline \multirow[t]{2}{*}{$\mathrm{P}$} & $\begin{array}{ll}0 & 1\end{array}$ & $-\quad 0$ & \\
\hline & \multicolumn{2}{|c|}{$1.900000000000000 \mathrm{E}-02$} & 1.00000000000000 \\
\hline \multirow[t]{4}{*}{$\mathrm{D}$} & $0 \quad 3$ & $-\quad 1$ & \\
\hline & \multicolumn{2}{|c|}{28.9500000000000} & $2.826900000000000 \mathrm{E}-02$ \\
\hline & \multicolumn{2}{|c|}{7.70800000000000} & 0.150890000000000 \\
\hline & \multicolumn{2}{|c|}{2.49500000000000} & 0.370373000000000 \\
\hline \multirow[t]{2}{*}{$\mathrm{D}$} & $\begin{array}{ll}0 & 1\end{array}$ & $-\quad 1$ & \\
\hline & \multicolumn{2}{|c|}{0.765500000000000} & 1.000000000000000 \\
\hline $\mathrm{D}$ & $\begin{array}{ll}0 & 1\end{array}$ & $-\quad 0$ & \\
\hline & \multicolumn{2}{|c|}{0.188900000000000} & 1.00000000000000 \\
\hline $\mathrm{D}$ & $\begin{array}{ll}-1 & 1\end{array}$ & $-\quad 0$ & \\
\hline & \multicolumn{2}{|c|}{0.044000000000000} & 1.00000000000000 \\
\hline \multicolumn{4}{|c|}{ 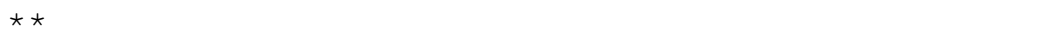 } \\
\hline $\mathrm{Cr}$ & \multicolumn{2}{|l|}{210} & \\
\hline \multicolumn{4}{|c|}{ D_AND_UP } \\
\hline 1 & \multicolumn{3}{|c|}{-10.00000000} \\
\hline 2 & 59.54875970 & -57.9409 & 980 \\
\hline 2 & 14.82659660 & -10.2623 & 850 \\
\hline & & & \\
\hline 0 & 100.02606530 & 3.000 & 0000 \\
\hline 1 & 67.84681240 & 25.253 & 5990 \\
\hline 2 & 34.67710060 & 210.6472 & 660 \\
\hline 2 & 7.67570050 & 235.2819 & 870 \\
\hline 2 & 7.20774270 & -152.5343 & 610 \\
\hline & & & \\
\hline 0 & 47.97233210 & 5.000 & 0000 \\
\hline 1 & 59.62248730 & 5.639 & 2660 \\
\hline 2 & 26.04326000 & 164.8362 & 980 \\
\hline 2 & 6.55826920 & 106.0168 & 180 \\
\hline 2 & 6.12682360 & -69.8585 & 680 \\
\hline & & & \\
\hline $\mathrm{Mr}$ & & & \\
\hline S & $0 \quad 3$ & $-\quad 1$ & \\
\hline & 5.9140000000 & 0000 & -0.374541000000000 \\
\hline & 1.6050000000 & 00000 & 0.769388000000000 \\
\hline & 0.62600000000 & 00000 & 0.477607000000000 \\
\hline S & $0 \quad 2$ & $-\quad 1$ & \\
\hline & 1.6050000000 & 00000 & $-3.779600000000000 \mathrm{E}-02$ \\
\hline & 0.62600000000 & 00000 & -0.141155000000000 \\
\hline S & $\begin{array}{ll}0 & 1\end{array}$ & $-\quad 0$ & \\
\hline & 0.11150000000 & 00000 & 1.000000000000000 \\
\hline S & $\begin{array}{ll}0 & 1\end{array}$ & $-\quad 0$ & \\
\hline & 3.80000000000 & $00000 \mathrm{E}-02$ & 1.00000000000000 \\
\hline $\mathrm{P}$ & $0 \quad 2$ & -1 & \\
\hline & 18.200000000 & 00000 & $-4.465000000000000 \mathrm{E}-02$ \\
\hline & 2.1410000000 & 00000 & 0.624073000000000 \\
\hline $\mathrm{P}$ & $\begin{array}{ll}0 & 1\end{array}$ & $-\quad 1$ & \\
\hline & 0.70090000000 & 00000 & 1.000000000000000 \\
\hline $\mathrm{P}$ & $\begin{array}{ll}0 & 1\end{array}$ & $-\quad 0$ & \\
\hline & 6.90000000000 & $0001 \mathrm{E}-02$ & 1.00000000000000 \\
\hline $\mathrm{P}$ & $\begin{array}{ll}0 & 1\end{array}$ & $-\quad 0$ & \\
\hline & 2.10000000000 & $0000 \mathrm{E}-02$ & 1.00000000000000 \\
\hline
\end{tabular}




\begin{tabular}{|c|c|c|c|}
\hline & $0 \quad 3$ & $-\quad 2$ & \\
\hline & 32.27000000 & 0000 & $2.895500000000000 \mathrm{E}-02$ \\
\hline & 8.875000000 & 0000 & 0.149285000000000 \\
\hline & 2.890000000 & 0000 & 0.372495000000000 \\
\hline & $\begin{array}{ll}0 & 1\end{array}$ & $-\quad 2$ & \\
\hline & 0.8761000000 & 0000 & 1.000000000000000 \\
\hline & $\begin{array}{ll}0 & 1\end{array}$ & $-\quad 0$ & \\
\hline & 0.21200000000 & 0000 & 1.00000000000000 \\
\hline & $\begin{array}{ll}-1 & 1\end{array}$ & $-\quad 0$ & \\
\hline & 0.0490000000 & 0000 & 1.00000000000000 \\
\hline & & & \\
\hline & 210 & & \\
\hline & ND_UP & & \\
\hline & $3 \overline{5} 7.39144690$ & -10.000 & 0000 \\
\hline & 64.64773890 & -60.2990 & 870 \\
\hline & 16.09608330 & -10.4217 & 340 \\
\hline & & & \\
\hline & 107.41272150 & 3.000 & 0000 \\
\hline & 111.49589730 & 16.259 & 8190 \\
\hline 2 & 46.55683460 & 276.9373 & 280 \\
\hline 2 & 8.36881350 & 241.3174 & 420 \\
\hline 2 & 7.72374890 & -146.4635 & 290 \\
\hline & & & \\
\hline 0 & 80.04151030 & 5.000 & 0000 \\
\hline 1 & 105.60436460 & 5.758 & 7560 \\
\hline 2 & 40.83004660 & 285.2918 & 540 \\
\hline 2 & 8.00984570 & 143.4222 & 470 \\
\hline 2 & 7.33909280 & -88.7031 & 510 \\
\hline & & & \\
\hline & & & \\
\hline & $0 \quad 3$ & $-\quad 1$ & \\
\hline & 6.422000000 & 0000 & -0.390482000000000 \\
\hline & 1.826000000 & 0000 & 0.767597000000000 \\
\hline & 0.71350000000 & 0000 & 0.492061000000000 \\
\hline & $\begin{array}{ll}0 & 2\end{array}$ & $-\quad 1$ & \\
\hline & 1.826000000 & 0000 & $-3.645700000000000 \mathrm{E}-02$ \\
\hline & 0.7135000000 & 0000 & -0.123931000000000 \\
\hline & $\begin{array}{ll}0 & 1\end{array}$ & $-\quad 0$ & \\
\hline & 0.1021000000 & 0000 & 1.000000000000000 \\
\hline & $\begin{array}{ll}0 & 1\end{array}$ & $-\quad 0$ & \\
\hline & 3.6300000000 & $0000 \mathrm{E}-02$ & 1.00000000000000 \\
\hline & $\begin{array}{ll}0 & 2\end{array}$ & -1 & \\
\hline & 19.48000000 & 0000 & $-4.688900000000000 \mathrm{E}-02$ \\
\hline & 2.389000000 & 0000 & 0.623034000000000 \\
\hline & $\begin{array}{ll}0 & 1\end{array}$ & $-\quad 1$ & \\
\hline & 0.77950000000 & 0000 & 1.000000000000000 \\
\hline 1 & $\begin{array}{ll}0 & 1\end{array}$ & $-\quad 0$ & \\
\hline & 7.40000000000 & $0000 E-02$ & 1.00000000000000 \\
\hline $\mathrm{P}$ & $\begin{array}{ll}0 & 1\end{array}$ & $-\quad 0$ & \\
\hline & 2.2000000000 & $0000 E-02$ & 1.00000000000000 \\
\hline $\mathrm{D}$ & $0 \quad 3$ & $-\quad 2$ & \\
\hline & 37.08000000 & 00000 & $2.829200000000000 \mathrm{E}-02$ \\
\hline & 10.10000000 & 0000 & 0.153707000000000 \\
\hline & 3.220000000 & 0000 & 0.385911000000000 \\
\hline$D$ & $\begin{array}{ll}0 & 1\end{array}$ & $-\quad 2$ & \\
\hline & 0.9628000000 & 0000 & 1.000000000000000 \\
\hline & $\begin{array}{ll}0 & 1\end{array}$ & $-\quad 0$ & \\
\hline
\end{tabular}




\begin{tabular}{|c|c|c|c|}
\hline & \multicolumn{2}{|c|}{0.226200000000000} & 1.00000000000000 \\
\hline D & $\begin{array}{ll}-1 & 1\end{array}$ & -0 & \\
\hline & \multicolumn{2}{|c|}{0.055000000000000} & 1.00000000000000 \\
\hline & & & \\
\hline & \multicolumn{2}{|l|}{210} & \\
\hline \multicolumn{4}{|c|}{ D_AND_UP } \\
\hline 1 & $3 \overline{9} 2.61497870$ & \multicolumn{2}{|c|}{-10.00000000} \\
\hline 2 & 71.17569790 & \multicolumn{2}{|c|}{-63.26675180} \\
\hline$z$ & 17.73202810 & \multicolumn{2}{|c|}{-10.96133380} \\
\hline & \\
\hline 0 & 126.05718950 & \multicolumn{2}{|c|}{3.00000000} \\
\hline 1 & 138.12642510 & \multicolumn{2}{|c|}{18.17291370} \\
\hline 2 & 54.20988580 & \multicolumn{2}{|c|}{339.12311640} \\
\hline 2 & 9.28379660 & \multicolumn{2}{|c|}{317.10680120} \\
\hline 2 & 8.62890820 & \multicolumn{2}{|c|}{-207.34216490} \\
\hline & \\
\hline 0 & 83.17594900 & \multicolumn{2}{|c|}{5.00000000} \\
\hline 1 & 106.05599380 & \multicolumn{2}{|c|}{5.95359300} \\
\hline 2 & 42.82849370 & \multicolumn{2}{|c|}{294.26655270} \\
\hline 2 & 8.77018050 & \multicolumn{2}{|c|}{154.42446350} \\
\hline 2 & 8.03978180 & -95.3164 & 490 \\
\hline & & & \\
\hline $\mathrm{Cc}$ & & & \\
\hline S & $0 \quad 3$ & -2 & \\
\hline & 7.1760000000 & 00000 & -0.382974000000000 \\
\hline & 2.0090000000 & 00000 & 0.741670000000000 \\
\hline & 0.80550000000 & 00000 & 0.509692000000000 \\
\hline $\mathrm{S}$ & $0 \quad 2$ & $-\quad 2$ & \\
\hline & 2.0090000000 & 00000 & $-3.153700000000000 \mathrm{E}-02$ \\
\hline & 0.80550000000 & 00000 & -0.119853000000000 \\
\hline S & $0 \quad 1$ & -0 & \\
\hline & 0.10700000000 & 00000 & 1.000000000000000 \\
\hline S & $0 \quad 1$ & -0 & \\
\hline & 3.75000000000 & $00000 \mathrm{E}-02$ & 1.00000000000000 \\
\hline $\mathrm{P}$ & $0 \quad 2$ & -2 & \\
\hline & 21.390000000 & 00000 & $-4.790100000000000 \mathrm{E}-02$ \\
\hline & 2.6500000000 & 00000 & 0.620417000000000 \\
\hline $\mathrm{P}$ & $0 \quad 1$ & -2 & \\
\hline & 0.86190000000 & 00000 & 1.000000000000000 \\
\hline $\mathrm{P}$ & $0 \quad 1$ & $-\quad 0$ & \\
\hline & 8.00000000000 & $00000 \mathrm{E}-02$ & 1.00000000000000 \\
\hline $\mathrm{P}$ & $0 \quad 1$ & -0 & \\
\hline & 2.30000000000 & $00000 \mathrm{E}-02$ & 1.00000000000000 \\
\hline D & $0 \quad 3$ & -3 & \\
\hline & 39.250000000 & 00000 & $3.116900000000000 \mathrm{E}-02$ \\
\hline & 10.780000000 & 00000 & 0.163521000000000 \\
\hline & 3.4960000000 & 00000 & 0.390105000000000 \\
\hline D & $\begin{array}{ll}0 & 1\end{array}$ & -3 & \\
\hline & 1.0660000000 & 00000 & 1.000000000000000 \\
\hline D & $0 \quad 1$ & -0 & \\
\hline & 0.26060000000 & 00000 & 1.00000000000000 \\
\hline D & $\begin{array}{ll}-1 & 1\end{array}$ & -0 & \\
\hline & 0.06000000000 & 00000 & 1.00000000000000 \\
\hline & & & \\
\hline & 210 & & \\
\hline & JD_UP & & \\
\hline & $4 \overline{3} 4.10761040$ & -10.000 & 0000 \\
\hline
\end{tabular}




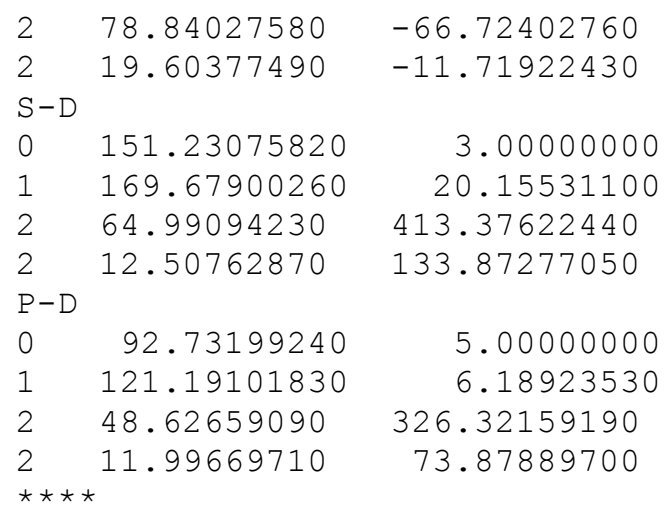

$\mathrm{Ni}$

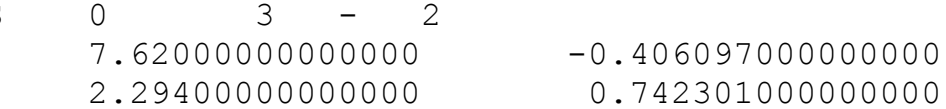

S $\quad 0 \quad 2 \quad-\quad 2$

$0.876000000000000 \quad 0.533018000000000$

$2.29400000000000 \quad-2.642700000000000 \mathrm{E}-02$

$0.876000000000000 \quad-0.121245000000000$

0

$0.115300000000000 \quad 1.000000000000000$

$\mathrm{S} \quad 0 \quad 1-0$

3.960000000000000E-02 1.00000000000000

$\mathrm{P} \quad 0 \quad 2-2$

$23.6600000000000 \quad-4.801900000000000 \mathrm{E}-02$

$2.89300000000000 \quad 0.624069000000000$

$\mathrm{P} \quad 0 \quad 1 \quad-\quad 2$

$0.943500000000000 \quad 1.000000000000000$

$\mathrm{P} \quad 0 \quad 1 \quad-0$

P $\quad 0 \quad 1 \quad-\quad 0$

$2.400000000000000 \mathrm{E}-02 \quad 1.00000000000000$

$\mathrm{D} \quad 0 \quad 3-3$

42.7200000000000

11.7600000000000

$3.236000000000000 \mathrm{E}-02$

3.81700000000000

0.169841000000000

0.396038000000000

$\begin{array}{lllll}\mathrm{D} & 0 & 1 & -\end{array}$

1.16900000000000

D $\quad 0 \quad 1 \quad-0$

1.000000000000000

0.283600000000000

$\begin{array}{lllll}\mathrm{D} & -1 & 1 & - & 0\end{array}$

0.065000000000000

1.00000000000000

1.00000000000000

$\star \star$

$\mathrm{Ni} \quad 2 \quad 10$

D AND UP

$\begin{array}{llr}1^{-} & 4 \overline{6} 9.93243310 & -10.00000000 \\ 2 & 85.42364110 & -69.40848050 \\ 2 & 21.26749840 & -12.09510200 \\ \text { S-D } & & \\ 0 & 162.16860970 & 3.00000000 \\ 1 & 176.53332320 & 22.02536180 \\ 2 & 68.95620100 & 443.01810880 \\ 2 & 13.57928380 & 145.56964110 \\ \text { P-D } & & \\ 0 & 69.01815060 & 5.00000000\end{array}$




\begin{tabular}{|c|c|c|c|}
\hline 1 & 275.59555960 & \multicolumn{2}{|c|}{4.98828240} \\
\hline 2 & 47.13154530 & \multicolumn{2}{|c|}{256.69458530} \\
\hline 2 & 12.98740750 & \multicolumn{2}{|c|}{78.47544500} \\
\hline \multicolumn{4}{|c|}{$\star \star \star \star$} \\
\hline \multicolumn{4}{|c|}{$\mathrm{Cu}$} \\
\hline & $0 \quad 3$ & $-\quad 2$ & \\
\hline & \multicolumn{2}{|c|}{8.17600000000000} & -0.419145000000000 \\
\hline & \multicolumn{2}{|c|}{2.56800000000000} & 0.735827000000000 \\
\hline & \multicolumn{2}{|c|}{0.958700000000000} & 0.552863000000000 \\
\hline S & $0 \quad 2$ & $-\quad 2$ & \\
\hline & \multicolumn{2}{|c|}{2.56800000000000} & $-2.276500000000000 \mathrm{E}-02$ \\
\hline & \multicolumn{2}{|c|}{0.958700000000000} & -0.117691000000000 \\
\hline S & $\begin{array}{ll}0 & 1\end{array}$ & $-\quad 0$ & \\
\hline & \multicolumn{2}{|c|}{0.115300000000000} & 1.000000000000000 \\
\hline \multirow[t]{2}{*}{ S } & $\begin{array}{ll}0 & 1\end{array}$ & $-\quad 0$ & \\
\hline & \multicolumn{2}{|c|}{$3.960000000000000 \mathrm{E}-02$} & 1.00000000000000 \\
\hline \multirow[t]{3}{*}{$\mathrm{P}$} & $0 \quad 2$ & -3 & \\
\hline & \multicolumn{2}{|c|}{25.6300000000000} & $-4.880200000000000 \mathrm{E}-02$ \\
\hline & \multicolumn{2}{|c|}{3.16600000000000} & 0.625804000000000 \\
\hline \multirow[t]{2}{*}{$\mathrm{P}$} & $0 \quad 1$ & $-\quad 3$ & \\
\hline & \multicolumn{2}{|c|}{1.02300000000000} & 1.000000000000000 \\
\hline $\mathrm{P}$ & $\begin{array}{ll}0 & 1\end{array}$ & $-\quad 0$ & \\
\hline & 8.59999999999 & $9999 E-02$ & 1.00000000000000 \\
\hline $\mathrm{P}$ & $\begin{array}{ll}0 & 1\end{array}$ & $-\quad 0$ & \\
\hline & 2.40000000000 & $0000 E-02$ & 1.00000000000000 \\
\hline D & $0 \quad 3$ & -3 & \\
\hline & 41.340000000 & 0000 & $4.075400000000000 \mathrm{E}-02$ \\
\hline & 11.420000000 & 0000 & 0.195077000000000 \\
\hline & 3.8390000000 & 0000 & 0.397458000000000 \\
\hline D & $0 \quad 1$ & -3 & \\
\hline & 1.2300000000 & 0000 & 1.000000000000000 \\
\hline D & $\begin{array}{ll}0 & 1\end{array}$ & $-\quad 1$ & \\
\hline & 0.31020000000 & 0000 & 1.00000000000000 \\
\hline $\mathrm{D}$ & $\begin{array}{ll}-1 & 1\end{array}$ & $-\quad 0$ & \\
\hline & 0.07000000000 & 0000 & 1.00000000000000 \\
\hline$\star x$ & & & \\
\hline $\mathrm{Cl}$ & 210 & & \\
\hline & JD_UP & & \\
\hline 1 & $5 \overline{1} 1.99517630$ & -10.000 & 0000 \\
\hline 2 & 93.28010740 & -72.5548 & 820 \\
\hline 2 & 23.22066690 & -12.7450 & 310 \\
\hline & & & \\
\hline 0 & 173.11808540 & 3.000 & 0000 \\
\hline 1 & 185.24198860 & 23.835 & 8250 \\
\hline 2 & 73.15178470 & 473.8930 & 880 \\
\hline 2 & 14.68841570 & 157.6345 & 230 \\
\hline & & & \\
\hline 0 & 100.71913690 & 5.000 & 0000 \\
\hline 1 & 130.83456650 & 6.499 & 9360 \\
\hline 2 & 53.86837200 & 351.4605 & 950 \\
\hline 2 & 14.09894690 & 85.5016 & 360 \\
\hline & & & \\
\hline Y & & & \\
\hline S & $0 \quad 3$ & $\begin{array}{ll}- & 1\end{array}$ & \\
\hline & 1.7510000000 & 0000 & -1.15418200000000 \\
\hline & 1.1430000000 & 0000 & 1.28790200000000 \\
\hline & 0.35810000000 & 0000 & 0.712344000000000 \\
\hline
\end{tabular}




\begin{tabular}{|c|c|c|c|}
\hline S & \multicolumn{2}{|c|}{$\begin{array}{l}1.14300000000000 \\
0.358100000000000\end{array}$} & $\begin{array}{l}-2.215900000000000 \mathrm{E}-02 \\
-0.243036000000000\end{array}$ \\
\hline \multirow[t]{2}{*}{ S } & $\begin{array}{ll}0 & 1\end{array}$ & $-\quad 0$ & \\
\hline & \multicolumn{2}{|c|}{0.105800000000000} & 1.000000000000000 \\
\hline \multirow[t]{2}{*}{$S$} & $\begin{array}{ll}0 & 1\end{array}$ & $-\quad 0$ & \\
\hline & \multicolumn{2}{|c|}{$3.180000000000000 \mathrm{E}-02$} & 1.00000000000000 \\
\hline & $0 \quad 2$ & -1 & \\
\hline & \multicolumn{2}{|c|}{3.88400000000000} & $\begin{array}{l}-8.128299999999999 \mathrm{E}-02 \\
0.669244000000000\end{array}$ \\
\hline & $\begin{array}{ll}0 & 1\end{array}$ & $-\quad 1$ & \\
\hline & \multicolumn{2}{|c|}{0.289000000000000} & 1.000000000000000 \\
\hline & $0 \quad 1$ & -0 & \\
\hline & \multicolumn{2}{|c|}{$6.290000000000000 \mathrm{E}-02$} & 1.00000000000000 \\
\hline & $0 \quad 1$ & -0 & \\
\hline & \multicolumn{2}{|c|}{$2.230000000000000 \mathrm{E}-02$} & 1.00000000000000 \\
\hline & $0 \quad 2$ & -1 & \\
\hline & \multicolumn{2}{|c|}{1.52300000000000} & $8.880000000000000 \mathrm{E}-02$ \\
\hline & \multicolumn{2}{|c|}{0.563400000000000} & 0.377058000000000 \\
\hline & $\begin{array}{ll}0 & 1\end{array}$ & $\begin{array}{ll}- & 0\end{array}$ & \\
\hline & \multicolumn{2}{|c|}{0.183400000000000} & 1.000000000000000 \\
\hline D & $\begin{array}{cc}0 & 1 \\
5.6900000000\end{array}$ & $\begin{array}{c}- \\
- \\
00000 \mathrm{E}-02\end{array}$ & 1.00000000000000 \\
\hline & \multirow{2}{*}{\multicolumn{2}{|c|}{0.017000000000000}} & \\
\hline & & & \\
\hline & \multicolumn{2}{|l|}{328} & \\
\hline \multicolumn{4}{|c|}{ F_AND_UP } \\
\hline & $5 \overline{7} 8.43103490$ & -0.040 & 8170 \\
\hline & 152.77920040 & -20.619 & 3440 \\
\hline & 44.93015240 & $-116 \cdot 7522$ & 790 \\
\hline & 11.45879180 & -43.7975 & 060 \\
\hline & 3.75232670 & -5.4247 & 090 \\
\hline & & & \\
\hline & 59.47151140 & 2.980 & 3390 \\
\hline & 17.21735530 & 34.783 & 6760 \\
\hline & 18.47970930 & 28.8453 & 460 \\
\hline 2 & 4.32761920 & 64.7642 & 880 \\
\hline & & & \\
\hline 0 & 45.72710050 & 4.988 & 7830 \\
\hline 1 & 49.45958860 & 19.650 & 5640 \\
\hline 2 & 18.99523730 & 194.0943 & 810 \\
\hline 2 & 3.66031930 & 43.1349 & 690 \\
\hline & & & \\
\hline 0 & 62.82684350 & 3.006 & 6470 \\
\hline 1 & 31.88979040 & 25.987 & 2500 \\
\hline 2 & 18.36465720 & 85.7172 & 970 \\
\hline 2 & 7.30624000 & 48.7792 & 680 \\
\hline 2 & 2.40516350 & 11.4535 & 040 \\
\hline & & & \\
\hline Z & & & \\
\hline$S$ & $0 \quad 3$ & -1 & \\
\hline & 1.976000000 & 00000 & -0.914787000000000 \\
\hline & 1.154000000 & 00000 & 1.08679100000000 \\
\hline & 0.3910000000 & 00000 & 0.679775000000000 \\
\hline S & $\begin{array}{lc}0 & 2 \\
1.154000000\end{array}$ & ${ }^{-}{ }^{1}$ & $-3.972500000000000 \mathrm{E}-02$ \\
\hline
\end{tabular}




\begin{tabular}{|c|c|c|c|}
\hline \multicolumn{3}{|c|}{0.391000000000000} & \multirow[t]{2}{*}{-0.206641000000000} \\
\hline \multirow[t]{2}{*}{$S$} & $\begin{array}{ll}0 & 1\end{array}$ & $-\quad 0$ & \\
\hline & 0.10010000000 & 00000 & 1.000000000000000 \\
\hline \multirow[t]{2}{*}{ S } & $\begin{array}{ll}0 & 1\end{array}$ & $-\quad 0$ & \\
\hline & 3.34000000000 & $00000 E-02$ & 1.00000000000000 \\
\hline \multirow[t]{3}{*}{$\mathrm{P}$} & $0 \quad 2$ & -1 & \\
\hline & 4.1920000000 & 00000 & $-9.350899999999999 E-02$ \\
\hline & 0.87640000000 & 00000 & 0.672996000000000 \\
\hline \multirow[t]{2}{*}{$\mathrm{P}$} & $\begin{array}{ll}0 & 1\end{array}$ & $-\quad 1$ & \\
\hline & 0.32630000000 & 00000 & 1.000000000000000 \\
\hline \multirow[t]{2}{*}{$\mathrm{P}$} & $\begin{array}{ll}0 & 1\end{array}$ & $-\quad 0$ & \\
\hline & 7.24000000000 & $00001 E-02$ & 1.00000000000000 \\
\hline \multirow[t]{2}{*}{$\mathrm{P}$} & $\begin{array}{ll}0 & 1\end{array}$ & $-\quad 0$ & \\
\hline & 2.43000000000 & $00000 E-02$ & 1.00000000000000 \\
\hline \multirow[t]{3}{*}{$\mathrm{D}$} & $0 \quad 2$ & $-\quad 1$ & \\
\hline & 2.2690000000 & 00000 & $4.975500000000000 \mathrm{E}-02$ \\
\hline & 0.78550000000 & 00000 & 0.392753000000000 \\
\hline \multirow[t]{2}{*}{$\mathrm{D}$} & $\begin{array}{ll}0 & 1\end{array}$ & $-\quad 1$ & \\
\hline & 0.26150000000 & 00000 & 1.000000000000000 \\
\hline \multirow[t]{2}{*}{$\mathrm{D}$} & $\begin{array}{ll}0 & 1\end{array}$ & $-\quad 0$ & \\
\hline & 8.01999999999 & $99999 \mathrm{E}-02$ & 1.00000000000000 \\
\hline \multirow[t]{2}{*}{ D } & $\begin{array}{ll}-1 & 1\end{array}$ & $-\quad 0$ & \\
\hline & 0.02300000000 & 00000 & 1.00000000000000 \\
\hline \multicolumn{4}{|c|}{ 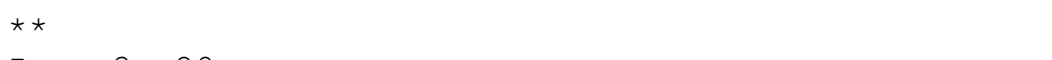 } \\
\hline & & & \\
\hline \multicolumn{4}{|c|}{$\begin{array}{l}Z r \quad 3 \quad 28 \\
F \text { AND UP }\end{array}$} \\
\hline 0 & $6 \overline{4} 5.93218730$ & \multicolumn{2}{|c|}{-0.04258430} \\
\hline 1 & 134.75474010 & \multicolumn{2}{|c|}{-20.22224090} \\
\hline 2 & 42.30746190 & \multicolumn{2}{|c|}{-101.86951720} \\
\hline 2 & 12.00032270 & \multicolumn{2}{|c|}{-41.61957840} \\
\hline 2 & 4.12604540 & -4.6986 & 600 \\
\hline$S-$ & & & \\
\hline 0 & 117.62518620 & 2.791 & 5590 \\
\hline 1 & 22.96460890 & 41.948 & 4590 \\
\hline 2 & 4.52252980 & 67.7271 & 660 \\
\hline $\mathrm{P}-$ & & & \\
\hline 0 & 47.19531450 & 4.991 & 1440 \\
\hline 1 & 48.03560330 & 20.719 & 1720 \\
\hline 2 & 19.45414560 & 195.5867 & 580 \\
\hline 2 & 4.05128750 & 48.2877 & 760 \\
\hline D- & & & \\
\hline 0 & 79.90739830 & 3.004 & 2260 \\
\hline 1 & 45.82637980 & 25.937 & 9890 \\
\hline 2 & 26.99035220 & 125.1244 & 340 \\
\hline 2 & 9.68357180 & 70.7634 & 220 \\
\hline 2 & 2.79956660 & 15.0492 & 220 \\
\hline & & & \\
\hline $\mathrm{Nk}$ & & & \\
\hline S & $0 \quad 3$ & $-\quad 1$ & \\
\hline & 2.1820000000 & 00000 & -0.882042000000000 \\
\hline & 1.2090000000 & 00000 & 1.10058900000000 \\
\hline & 0.41650000000 & 00000 & 0.630462000000000 \\
\hline S & $\begin{array}{ll}0 & 2\end{array}$ & $-\quad 1$ & \\
\hline & 1.2090000000 & 00000 & $-4.221500000000000 \mathrm{E}-02$ \\
\hline & 0.41650000000 & 00000 & -0.242823000000000 \\
\hline$S$ & $\begin{array}{ll}0 & 1\end{array}$ & $-\quad 0$ & \\
\hline & 0.14540000000 & 00000 & 1.000000000000000 \\
\hline
\end{tabular}




\begin{tabular}{|c|c|c|c|}
\hline & 0 & 0 & \\
\hline & 3.92000000000 & $0000 E-02$ & 1.00000000000000 \\
\hline & $0 \quad 2$ & $-\quad 1$ & \\
\hline & 4.5190000000 & 0000 & $-8.119800000000001 \mathrm{E}-02$ \\
\hline & 0.94060000000 & 0000 & 0.694956000000000 \\
\hline & $\begin{array}{ll}0 & 1\end{array}$ & $-\quad 1$ & \\
\hline & 0.34920000000 & 0000 & 1.000000000000000 \\
\hline & $\begin{array}{ll}0 & 1\end{array}$ & $-\quad 0$ & \\
\hline & 7.52000000000 & $0000 E-02$ & 1.00000000000000 \\
\hline & $\begin{array}{ll}0 & 1\end{array}$ & $-\quad 0$ & \\
\hline & 2.47000000000 & $0000 E-02$ & 1.00000000000000 \\
\hline & $0 \quad 2$ & -1 & \\
\hline & 3.4660000000 & 0000 & $2.634300000000000 \mathrm{E}-02$ \\
\hline & 0.99380000000 & 0000 & 0.403029000000000 \\
\hline & $\begin{array}{ll}0 & 1\end{array}$ & $-\quad 1$ & \\
\hline & 0.33500000000 & 0000 & 1.000000000000000 \\
\hline & $\begin{array}{ll}0 & 1\end{array}$ & $-\quad 0$ & \\
\hline & 0.10240000000 & 0000 & 1.00000000000000 \\
\hline & $\begin{array}{ll}-1 & 1\end{array}$ & $-\quad 0$ & \\
\hline & 0.02800000000 & 0000 & 1.00000000000000 \\
\hline & & & \\
\hline & 328 & & \\
\hline & ID_UP & & \\
\hline & $3 \overline{4} 2.96384050$ & -0.04 & 4010 \\
\hline & 139.93080140 & -20.05 & 1000 \\
\hline & 43.35234050 & -103.724 & 210 \\
\hline , & 12.44485330 & -41.045 & 650 \\
\hline ? & 4.32048370 & -4.204 & 190 \\
\hline & & & \\
\hline 0 & 112.86306170 & 2.79 & 6690 \\
\hline L & 22.96786760 & 42.88 & 7860 \\
\hline 2 & 4.93406730 & 75.187 & 660 \\
\hline & & & \\
\hline 0 & 63.68019630 & 4.94 & 8460 \\
\hline 1 & 23.09124290 & 24.83 & 3760 \\
\hline 2 & 24.42836470 & 137.918 & 920 \\
\hline 2 & 4.23100900 & 50.977 & 710 \\
\hline & & & \\
\hline 0 & 99.33596360 & 3.00 & 0590 \\
\hline 1 & 64.05864720 & 25.53 & 4580 \\
\hline 2 & 37.08910110 & $178.959^{\circ}$ & 520 \\
\hline 2 & 12.07085740 & $92.957^{\circ}$ & 900 \\
\hline 2 & 3.15753230 & 18.474 & 560 \\
\hline & & & \\
\hline$\sqrt{1}$ & & & \\
\hline & $0 \quad 3$ & $\begin{array}{ll}- & 1\end{array}$ & \\
\hline & 2.3610000000 & 0000 & -0.910746000000000 \\
\hline & 1.3090000000 & 0000 & 1.14621400000000 \\
\hline & 0.45000000000 & 0000 & 0.610233000000000 \\
\hline (2) & $0 \quad 2$ & $-\quad 1$ & \\
\hline & 1.3090000000 & 0000 & $-4.486400000000000 \mathrm{E}-02$ \\
\hline & 0.45000000000 & 0000 & -0.247454000000000 \\
\hline S & $\begin{array}{ll}0 & 1\end{array}$ & $-\quad 0$ & \\
\hline & 0.16810000000 & 0000 & 1.000000000000000 \\
\hline 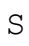 & $\begin{array}{ll}0 & 1\end{array}$ & $-\quad 0$ & \\
\hline & 4.23000000000 & $0000 E-02$ & 1.00000000000000 \\
\hline & $0 \quad 2$ & $-\quad 1$ & \\
\hline
\end{tabular}




\begin{tabular}{|c|c|c|c|}
\hline & \multicolumn{2}{|c|}{$\begin{array}{l}4.89500000000000 \\
1.04400000000000\end{array}$} & $\begin{array}{l}-9.029300000000000 E-02 \\
0.700158000000000\end{array}$ \\
\hline \multirow[t]{2}{*}{$\mathrm{P}$} & $\begin{array}{ll}0 & 1\end{array}$ & $\begin{array}{ll}- & 1\end{array}$ & \\
\hline & \multicolumn{2}{|c|}{0.387700000000000} & 1.000000000000000 \\
\hline \multirow[t]{2}{*}{$\mathrm{P}$} & $\begin{array}{ll}0 & 1\end{array}$ & $-\quad 0$ & \\
\hline & \multicolumn{2}{|c|}{$7.800000000000000 \mathrm{E}-02$} & 1.00000000000000 \\
\hline \multirow[t]{2}{*}{$\mathrm{P}$} & $\begin{array}{ll}0 & 1\end{array}$ & $-\quad 0$ & \\
\hline & \multicolumn{2}{|c|}{$2.470000000000000 \mathrm{E}-02$} & 1.00000000000000 \\
\hline \multirow[t]{3}{*}{ D } & $0 \quad 2$ & $-\quad 1$ & \\
\hline & \multicolumn{2}{|c|}{2.99300000000000} & $4.526600000000000 \mathrm{E}-02$ \\
\hline & \multicolumn{2}{|c|}{1.06300000000000} & 0.429772000000000 \\
\hline \multirow[t]{2}{*}{ D } & $\begin{array}{ll}0 & 1\end{array}$ & $-\quad 1$ & \\
\hline & \multicolumn{2}{|c|}{0.372100000000000} & 1.000000000000000 \\
\hline \multirow[t]{2}{*}{ D } & $\begin{array}{ll}0 & 1\end{array}$ & $-\quad 0$ & \\
\hline & \multicolumn{2}{|c|}{0.117800000000000} & 1.00000000000000 \\
\hline \multirow[t]{2}{*}{ D } & $\begin{array}{ll}-1 & 1\end{array}$ & $-\quad 0$ & \\
\hline & \multicolumn{2}{|c|}{0.034000000000000} & 1.00000000000000 \\
\hline \multicolumn{4}{|c|}{ 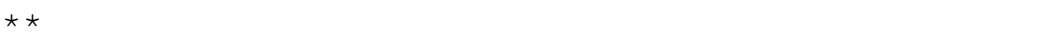 } \\
\hline $\mathrm{Mc}$ & \multicolumn{2}{|l|}{328} & \\
\hline \multicolumn{4}{|c|}{ F_AND UP } \\
\hline 0 & $5 \overline{3} 7.96678070$ & -0.046 & 4920 \\
\hline 1 & 147.89829380 & -20.208 & 840 \\
\hline 2 & 45.73588980 & -106.2116 & 20 \\
\hline 2 & 13.29114670 & -41.8107 & 580 \\
\hline 2 & 4.70599610 & -4.2054 & 030 \\
\hline & & & \\
\hline 0 & 110.29917600 & 2.806 & 7170 \\
\hline 1 & 23.20146450 & 44.516 & 120 \\
\hline 2 & 5.35301310 & 82.7785 & 270 \\
\hline & & & \\
\hline 0 & 63.29013970 & 4.942 & 3760 \\
\hline 1 & 23.33153020 & 25.860 & 9760 \\
\hline 2 & 24.67594230 & 132.4708 & 420 \\
\hline 2 & 4.64930400 & 57.3149 & 940 \\
\hline & & & \\
\hline 0 & 104.48399770 & 3.005 & 5910 \\
\hline 1 & 66.23072450 & 26.363 & 3510 \\
\hline 2 & 39.12831760 & 183.3894 & 990 \\
\hline 2 & 13.11644370 & 98.4453 & 580 \\
\hline 2 & 3.62802630 & 22.4901 & 770 \\
\hline & & & \\
\hline $\mathrm{T}$ & & & \\
\hline S & $0 \quad 3$ & $\begin{array}{ll}- & 1\end{array}$ & \\
\hline & 2.3420000000 & 0000 & -1.48655100000000 \\
\hline & 1.6340000000 & 0000 & 1.67009400000000 \\
\hline & 0.50940000000 & 0000 & 0.657337000000000 \\
\hline$S$ & $0 \quad 2$ & $-\quad 1$ & \\
\hline & 1.6340000000 & 0000 & $-4.036600000000000 E-02$ \\
\hline & 0.50940000000 & 0000 & -0.216478000000000 \\
\hline$S$ & $\begin{array}{ll}0 & 1\end{array}$ & $-\quad 0$ & \\
\hline & 0.17060000000 & 0000 & 1.000000000000000 \\
\hline$S$ & $\begin{array}{ll}0 & 1\end{array}$ & $-\quad 0$ & \\
\hline & 4.25000000000 & $0000 E-02$ & 0.860266000000000 \\
\hline $\mathrm{P}$ & $0 \quad 2$ & $-\quad 1$ & \\
\hline & 5.2780000000 & 0000 & $-9.896600000000000 \mathrm{E}-02$ \\
\hline & 1.1560000000 & 0000 & 0.704057000000000 \\
\hline $\mathrm{P}$ & $0 \quad 1$ & $-\quad 1$ & \\
\hline
\end{tabular}




\begin{tabular}{|c|c|c|c|}
\hline \multicolumn{3}{|c|}{0.432000000000000} & \multirow[t]{2}{*}{1.000000000000000} \\
\hline \multirow[t]{2}{*}{$\mathrm{P}$} & $\begin{array}{ll}0 & 1\end{array}$ & $-\quad 0$ & \\
\hline & 8.95000000000 & $0000 E-02$ & 1.00000000000000 \\
\hline \multirow[t]{2}{*}{$\mathrm{P}$} & $\begin{array}{ll}0 & 1\end{array}$ & $-\quad 0$ & \\
\hline & 2.46000000000 & $0000 E-02$ & 1.00000000000000 \\
\hline \multirow[t]{3}{*}{$\mathrm{D}$} & $0 \quad 2$ & $-\quad 1$ & \\
\hline & 4.6320000000 & 0000 & $2.290600000000000 \mathrm{E}-02$ \\
\hline & 1.2790000000 & 0000 & 0.432430000000000 \\
\hline \multirow[t]{2}{*}{$\mathrm{D}$} & $\begin{array}{ll}0 & 1\end{array}$ & $-\quad 1$ & \\
\hline & 0.44250000000 & 0000 & 1.000000000000000 \\
\hline \multirow[t]{2}{*}{$\mathrm{D}$} & $\begin{array}{ll}0 & 1\end{array}$ & $-\quad 0$ & \\
\hline & 0.13640000000 & 0000 & 1.00000000000000 \\
\hline \multirow[t]{2}{*}{$\mathrm{D}$} & $\begin{array}{ll}-1 & 1\end{array}$ & $-\quad 0$ & \\
\hline & 0.03800000000 & 0000 & 1.00000000000000 \\
\hline \multicolumn{4}{|c|}{ 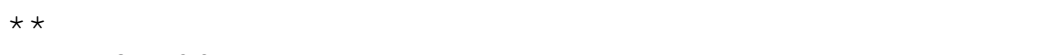 } \\
\hline $\mathrm{TC}$ & 328 & & \\
\hline \multicolumn{4}{|c|}{ F_AND_UP } \\
\hline 0 & $5 \overline{0} 1.11994090$ & \multicolumn{2}{|c|}{-0.04921150} \\
\hline 1 & 150.24688440 & \multicolumn{2}{|c|}{-20.14673080} \\
\hline 2 & 46.70979140 & \multicolumn{2}{|c|}{-105.57928800} \\
\hline 2 & 13.93390140 & \multicolumn{2}{|c|}{-41.59011970} \\
\hline 2 & 4.98540690 & \multicolumn{2}{|c|}{-3.94151110} \\
\hline \multicolumn{4}{|c|}{$S-F$} \\
\hline 0 & 108.17759950 & \multicolumn{2}{|c|}{2.82223740} \\
\hline 1 & 23.65943510 & \multicolumn{2}{|c|}{46.73307080} \\
\hline 2 & 5.78149770 & 90.5318 & 570 \\
\hline $\mathrm{P}-$ & & & \\
\hline 0 & 63.30620140 & 4.939 & 7820 \\
\hline 1 & 23.69355320 & 26.922 & 5310 \\
\hline 2 & 25.05258540 & 128.5075 & 960 \\
\hline 2 & 5.07170840 & 63.7490 & 500 \\
\hline & & & \\
\hline 0 & 102.77213970 & 2.992 & 9020 \\
\hline 1 & 45.67137560 & 28.182 & 3790 \\
\hline 2 & 48.26119970 & 89.0548 & 550 \\
\hline 2 & 15.70891150 & 121.1894 & 250 \\
\hline 2 & 3.94845760 & 25.5866 & 730 \\
\hline & & & \\
\hline Ru & & & \\
\hline S & $0 \quad 3$ & -1 & \\
\hline & 2.5650000000 & 0000 & -1.04037100000000 \\
\hline & 1.5080000000 & 0000 & 1.32852200000000 \\
\hline & 0.51290000000 & 0000 & 0.561194000000000 \\
\hline S & $\begin{array}{ll}0 & 2\end{array}$ & -1 & \\
\hline & 1.5080000000 & 0000 & $-5.959700000000000 \mathrm{E}-02$ \\
\hline & 0.51290000000 & 0000 & -0.151713000000000 \\
\hline S & $\begin{array}{ll}0 & 1\end{array}$ & $-\quad 0$ & \\
\hline & 0.13620000000 & 0000 & 1.000000000000000 \\
\hline S & $\begin{array}{ll}0 & 1\end{array}$ & $-\quad 0$ & \\
\hline & 4.17000000000 & $0000 \mathrm{E}-02$ & 1.00000000000000 \\
\hline $\mathrm{P}$ & $\begin{array}{ll}0 & 2\end{array}$ & -1 & \\
\hline & 4.8590000000 & 0000 & $-9.425799999999999 E-02$ \\
\hline & 1.2190000000 & 0000 & 0.740984000000000 \\
\hline $\mathrm{P}$ & $\begin{array}{ll}0 & 1\end{array}$ & $-\quad 1$ & \\
\hline & 0.44130000000 & 0000 & 1.000000000000000 \\
\hline $\mathrm{P}$ & $\begin{array}{ll}0 & 1\end{array}$ & $-\quad 0$ & \\
\hline & 8.30000000000 & $0000 \mathrm{E}-02$ & 1.00000000000000 \\
\hline
\end{tabular}




\begin{tabular}{|c|c|c|c|}
\hline \multirow[t]{2}{*}{$\mathrm{P}$} & $\begin{array}{ll}0 & 1\end{array}$ & $-\quad 0$ & \\
\hline & \multicolumn{2}{|c|}{$2.500000000000000 \mathrm{E}-02$} & 1.00000000000000 \\
\hline \multirow[t]{3}{*}{$\mathrm{D}$} & $0 \quad 2$ & $-\quad 1$ & \\
\hline & \multirow{2}{*}{\multicolumn{2}{|c|}{$\begin{array}{l}4.10500000000000 \\
1.37700000000000\end{array}$}} & $4.210600000000000 \mathrm{E}-02$ \\
\hline & & & 0.442552000000000 \\
\hline \multirow[t]{2}{*}{ D } & $\begin{array}{ll}0 & 1\end{array}$ & $-\quad 1$ & \\
\hline & \multicolumn{2}{|c|}{0.482800000000000} & 1.000000000000000 \\
\hline \multirow[t]{2}{*}{$\mathrm{D}$} & $\begin{array}{ll}0 & 1\end{array}$ & $-\quad 0$ & \\
\hline & \multicolumn{2}{|c|}{0.150100000000000} & 1.00000000000000 \\
\hline \multirow[t]{2}{*}{ D } & $\begin{array}{ll}-1 & 1\end{array}$ & $-\quad 0$ & \\
\hline & \multicolumn{2}{|c|}{0.042000000000000} & 1.00000000000000 \\
\hline \multicolumn{4}{|c|}{ - } \\
\hline Ru & \multicolumn{2}{|l|}{328} & \\
\hline \multicolumn{4}{|c|}{ F_AND_UP } \\
\hline 0 & $5 \overline{5} 4.37963030$ & \multicolumn{2}{|c|}{-0.05152700} \\
\hline 1 & 155.10668710 & \multicolumn{2}{|c|}{-20.18165360} \\
\hline 2 & 48.49762630 & \multicolumn{2}{|c|}{-105.99669150} \\
\hline 2 & 14.77015940 & \multicolumn{2}{|c|}{-42.21667880} \\
\hline 2 & 5.20773630 & \multicolumn{2}{|c|}{-3.76750240} \\
\hline \multicolumn{4}{|c|}{$S-F$} \\
\hline 0 & 66.71180600 & \multicolumn{2}{|c|}{2.95783440} \\
\hline 1 & 77.35036320 & \multicolumn{2}{|c|}{25.37487070} \\
\hline 2 & 18.35714450 & \multicolumn{2}{|c|}{536.12623720} \\
\hline 2 & 11.84047270 & \multicolumn{2}{|c|}{-651.20572210} \\
\hline 2 & 8.11794790 & \multicolumn{2}{|c|}{381.38169430} \\
\hline \multicolumn{4}{|c|}{$P-F$} \\
\hline 0 & 54.99379150 & \multicolumn{2}{|c|}{4.96515570} \\
\hline 1 & 13.93992120 & \multicolumn{2}{|c|}{23.88615010} \\
\hline 2 & 15.21182460 & \multicolumn{2}{|c|}{464.46313440} \\
\hline 2 & 10.54606910 & \multicolumn{2}{|c|}{-714.44517880} \\
\hline 2 & 7.55394860 & 377.550 & \\
\hline D- & & & \\
\hline 0 & 60.34445950 & 3.03 & 380 \\
\hline 1 & 45.21003050 & 23.29 & 230 \\
\hline 2 & 19.11900740 & 146.092 & \\
\hline 2 & 4.27120900 & 28.912 & \\
\hline
\end{tabular}

$\mathrm{Rh}$

$\begin{array}{lllll}\mathrm{S} & 0 & 3 & - & 1\end{array}$

2. 64600000000000

1.75100000000000

$-1.34877500000000$

0.571300000000000

$\begin{array}{lllll}S & 0 & 2 & - & 1\end{array}$

1.75100000000000

0.571300000000000

$0 \quad 1 \quad-$

0.143800000000000

$\mathrm{S} \quad 0 \quad 1 \quad-\quad 0$
4. $280000000000000 E-02$
1.00000000000000

1.60434700000000

0.589141000000000

$\begin{array}{lllll}\mathrm{P} & 0 & 2 & - & 1\end{array}$

5. 44000000000000

1.32900000000000

$\begin{array}{lllll}\mathrm{P} & 0 & 1 & - & 1\end{array}$

0.484500000000000

$-5.200800000000000 \mathrm{E}-02$

$-0.143435000000000$

1.000000000000000

$-9.844300000000000 E-02$

0.740899000000000

1.000000000000000

$\mathrm{P} \quad 0 \quad 1 \quad-\quad 0$

$8.690000000000001 \mathrm{E}-02 \quad 1.00000000000000$

$\mathrm{P} \quad 0 \quad 1 \quad-\quad 0$ 


\begin{tabular}{|c|c|c|c|}
\hline & \multicolumn{2}{|c|}{$2.570000000000000 \mathrm{E}-02$} & 1.00000000000000 \\
\hline \multirow[t]{3}{*}{$\mathrm{D}$} & $0 \quad 2$ & $-\quad 1$ & \\
\hline & \multirow{2}{*}{\multicolumn{2}{|c|}{$\begin{array}{l}3.66900000000000 \\
1.42300000000000\end{array}$}} & $6.704800000000000 \mathrm{E}-02$ \\
\hline & & & 0.455084000000000 \\
\hline \multirow[t]{2}{*}{$\mathrm{D}$} & $\begin{array}{ll}0 & 1\end{array}$ & $-\quad 1$ & \\
\hline & \multicolumn{2}{|c|}{0.509100000000000} & 1.000000000000000 \\
\hline \multirow[t]{2}{*}{$\mathrm{D}$} & $\begin{array}{ll}0 & 1\end{array}$ & $-\quad 0$ & \\
\hline & \multicolumn{2}{|c|}{0.161000000000000} & 1.00000000000000 \\
\hline \multirow[t]{2}{*}{$\mathrm{D}$} & $\begin{array}{ll}-1 & 1\end{array}$ & $-\quad 0$ & \\
\hline & \multicolumn{2}{|c|}{0.045000000000000} & 1.00000000000000 \\
\hline \multicolumn{4}{|c|}{ 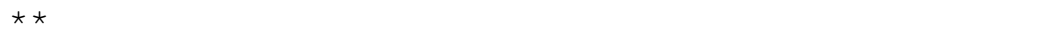 } \\
\hline $\mathrm{RH}$ & \multicolumn{2}{|l|}{328} & \\
\hline \multicolumn{4}{|c|}{ F_AND_UP } \\
\hline 0 & \multicolumn{2}{|l|}{$6 \overline{0} 0.32430320$} & 580 \\
\hline 1 & 157.69101760 & \multirow{2}{*}{\multicolumn{2}{|c|}{$\begin{array}{r}-20.13162820 \\
-105.36541210\end{array}$}} \\
\hline 2 & 49.88419950 & & -105.36541210 \\
\hline 2 & \multirow{2}{*}{$\begin{array}{r}15.59668950 \\
5.50992960\end{array}$} & -42.3274 & 00 \\
\hline 2 & & -3.6654 & 30 \\
\hline & & & \\
\hline 0 & 59.34425260 & 2.975 & 280 \\
\hline 1 & 83.74260610 & 25.123 & 060 \\
\hline 2 & 18.45302480 & 626.0926 & 50 \\
\hline 2 & 12.41946060 & -812.2549 & 50 \\
\hline 2 & 8.81729130 & 467.3729 & 00 \\
\hline & & & \\
\hline 0 & 53.43090680 & 4.953 & 130 \\
\hline 1 & 65.66718430 & 20.487 & 160 \\
\hline 2 & 16.83698620 & 598.0120 & 90 \\
\hline 2 & 11.30421360 & -718.4059 & 80 \\
\hline 2 & 8.03124440 & 382.8173 & 10 \\
\hline & & & \\
\hline 0 & 64.39936530 & 3.027 & 320 \\
\hline 1 & 43.46250530 & 24.752 & 160 \\
\hline 2 & 19.40203010 & 142.6844 & 90 \\
\hline 2 & 4.68793280 & 32.1406 & 80 \\
\hline & & & \\
\hline $\mathrm{Pc}$ & & & \\
\hline S & $0 \quad 3$ & $-\quad 1$ & \\
\hline & 2.7870000000 & 0000 & -1.60172800000000 \\
\hline & 1.9650000000 & 0000 & 1.84019500000000 \\
\hline & 0.62430000000 & 0000 & 0.603340000000000 \\
\hline S & $\begin{array}{ll}0 & 2\end{array}$ & $-\quad 1$ & \\
\hline & 1.9650000000 & 0000 & $-4.798200000000000 \mathrm{E}-02$ \\
\hline & 0.62430000000 & 0000 & -0.135579000000000 \\
\hline S & $\begin{array}{ll}0 & 1\end{array}$ & $-\quad 0$ & \\
\hline & 0.14960000000 & 0000 & 1.000000000000000 \\
\hline S & $\begin{array}{ll}0 & 1\end{array}$ & $-\quad 0$ & \\
\hline & 4.36000000000 & $0000 E-02$ & 1.00000000000000 \\
\hline $\mathrm{P}$ & $0 \quad 2$ & $-\quad 1$ & \\
\hline & 5.9990000000 & 0000 & -0.103179000000000 \\
\hline & 1.4430000000 & 0000 & 0.743447000000000 \\
\hline $\mathrm{P}$ & $\begin{array}{ll}0 & 1\end{array}$ & $\begin{array}{ll}- & 1\end{array}$ & \\
\hline & 0.52640000000 & 0000 & 1.000000000000000 \\
\hline $\mathrm{P}$ & $\begin{array}{ll}0 & 1\end{array}$ & $-\quad 0$ & \\
\hline & 8.98999999999 & $9999 E-02$ & 1.00000000000000 \\
\hline P & $\begin{array}{ll}0 & 1\end{array}$ & $-\quad 0$ & \\
\hline & 2.62000000000 & $0000 E-02$ & 1.00000000000000 \\
\hline
\end{tabular}




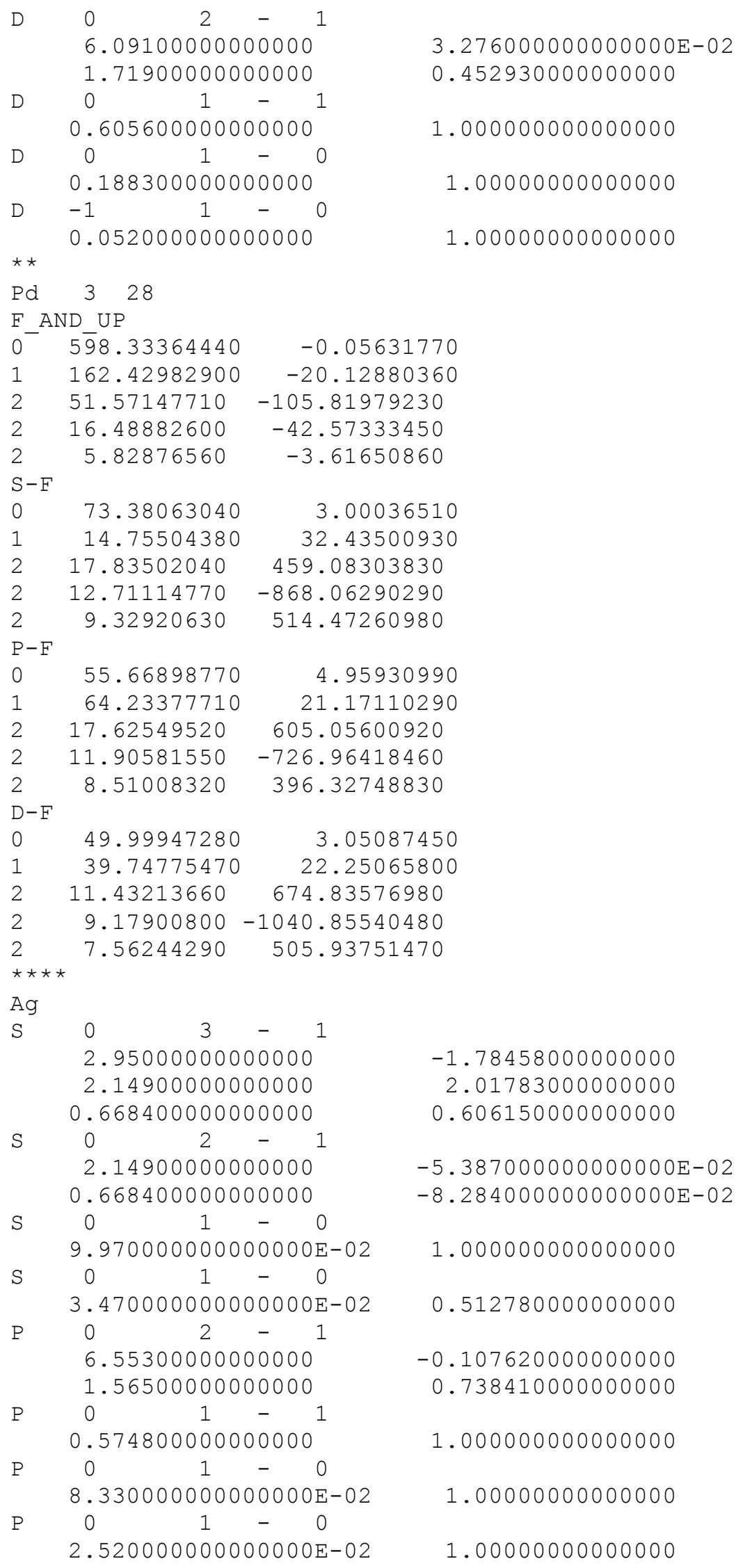




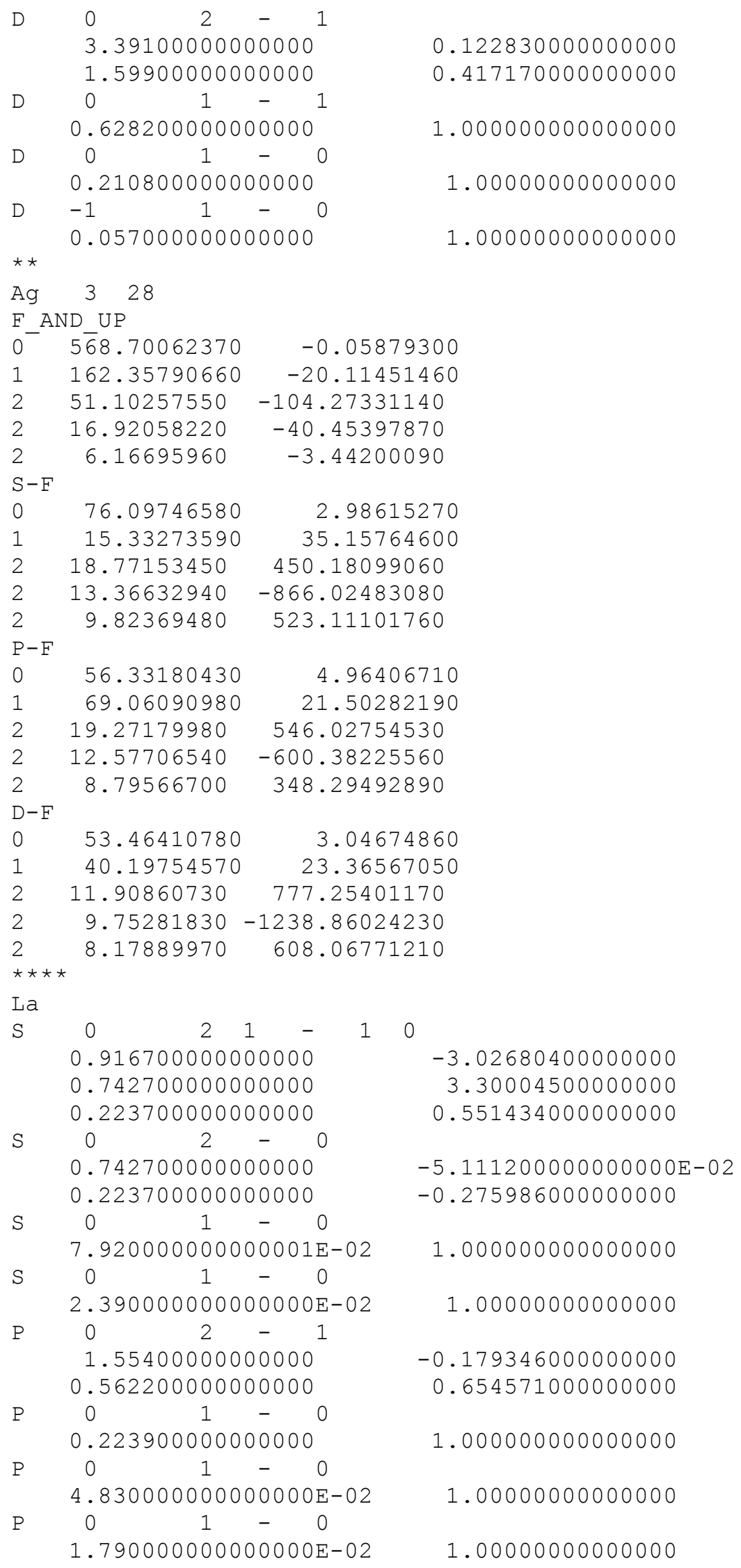




\begin{tabular}{|c|c|c|c|}
\hline \multirow[t]{2}{*}{$\mathrm{D}$} & $\begin{array}{ll}0 & 1\end{array}$ & -1 & \\
\hline & \multicolumn{2}{|c|}{0.452400000000000} & 0.365757000000000 \\
\hline \multirow[t]{2}{*}{$\mathrm{D}$} & $\begin{array}{ll}0 & 1\end{array}$ & $-\quad 0$ & \\
\hline & \multicolumn{2}{|c|}{0.160200000000000} & 0.535653000000000 \\
\hline \multirow[t]{2}{*}{ D } & $\begin{array}{ll}0 & 1\end{array}$ & $-\quad 0$ & \\
\hline & \multicolumn{2}{|c|}{$5.310000000000000 \mathrm{E}-02$} & 1.00000000000000 \\
\hline & $\begin{array}{ll}-1 & 1\end{array}$ & $-\quad 0$ & \\
\hline & \multicolumn{2}{|c|}{0.017000000000000} & 1.00000000000000 \\
\hline \multicolumn{4}{|c|}{ - } \\
\hline & \multicolumn{2}{|l|}{346} & \\
\hline \multicolumn{4}{|c|}{ F_AND_UP } \\
\hline & \multicolumn{3}{|c|}{-0.08647270} \\
\hline & 146.65714990 & \multicolumn{2}{|c|}{-33.51688030} \\
\hline & 42.35854130 & \multicolumn{2}{|c|}{-189.07098290} \\
\hline & 11.16475000 & \multicolumn{2}{|c|}{-54.24643670} \\
\hline & 3.75563430 & \multicolumn{2}{|c|}{-17.13513420} \\
\hline & 1.28718270 & \multicolumn{2}{|c|}{-1.80921850} \\
\hline \multicolumn{4}{|c|}{ 土. } \\
\hline & 125.68626650 & \multicolumn{2}{|c|}{2.88949430} \\
\hline & 37.57689550 & \multicolumn{2}{|c|}{61.95215920} \\
\hline 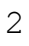 & 12.69548690 & 164.807 & 900 \\
\hline 2 & 2.62760820 & 55.695 & 090 \\
\hline & & & \\
\hline J & 179.17822470 & 4.59 & 4120 \\
\hline 1 & 42.61956610 & 71.67 & 7360 \\
\hline 2 & 13.41359570 & 175.896 & 960 \\
\hline 2 & 2.09503920 & 37.913 & 040 \\
\hline & & & \\
\hline 0 & 104.76820640 & 2.97 & 4960 \\
\hline 1 & 40.19303400 & 46.52 & 9460 \\
\hline 2 & 18.50507980 & 137.407 & 760 \\
\hline 2 & 5.80511850 & 63.494 & 830 \\
\hline 2 & 1.45832200 & 18.099 & 540 \\
\hline & & & \\
\hline & & & \\
\hline & $0 \quad 3$ & $-\quad 1$ & \\
\hline & 1.9500000000 & 0000 & -1.23343400000000 \\
\hline & 1.1830000000 & 0000 & 1.57817900000000 \\
\hline & 0.38970000000 & 0000 & 0.491661000000000 \\
\hline S & $0 \quad 2$ & $-\quad 1$ & \\
\hline & 1.1830000000 & 0000 & $-5.117900000000000 \mathrm{E}-02$ \\
\hline & 0.38970000000 & 0000 & -0.322442000000000 \\
\hline$S$ & $\begin{array}{ll}0 & 1\end{array}$ & $-\quad 0$ & \\
\hline & 0.1656000000 & 0000 & 1.000000000000000 \\
\hline$S$ & $\begin{array}{ll}0 & 1\end{array}$ & $-\quad 0$ & \\
\hline & 4.24000000000 & $0000 E-02$ & 1.00000000000000 \\
\hline $\mathrm{P}$ & $0 \quad 2$ & $-\quad 1$ & \\
\hline & 1.972000000 & 0000 & -0.625984000000000 \\
\hline & 1.3540000000 & 0000 & 1.03598600000000 \\
\hline F & $\begin{array}{ll}0 & 1\end{array}$ & $-\quad 0$ & \\
\hline & 0.4134000000 & 00000 & 1.000000000000000 \\
\hline $\mathrm{P}$ & $\begin{array}{ll}0 & 1\end{array}$ & $-\quad 0$ & \\
\hline & 8.0400000000 & $0000 E-02$ & 1.00000000000000 \\
\hline $\mathrm{P}$ & $\begin{array}{ll}0 & 1\end{array}$ & $-\quad 0$ & \\
\hline & 2.7400000000 & $0000 \mathrm{E}-02$ & 1.00000000000000 \\
\hline & $\begin{array}{ll}0 & 1\end{array}$ & $-\quad 1$ & \\
\hline & 0.8226000000 & 0000 & 1.000000000000000 \\
\hline
\end{tabular}




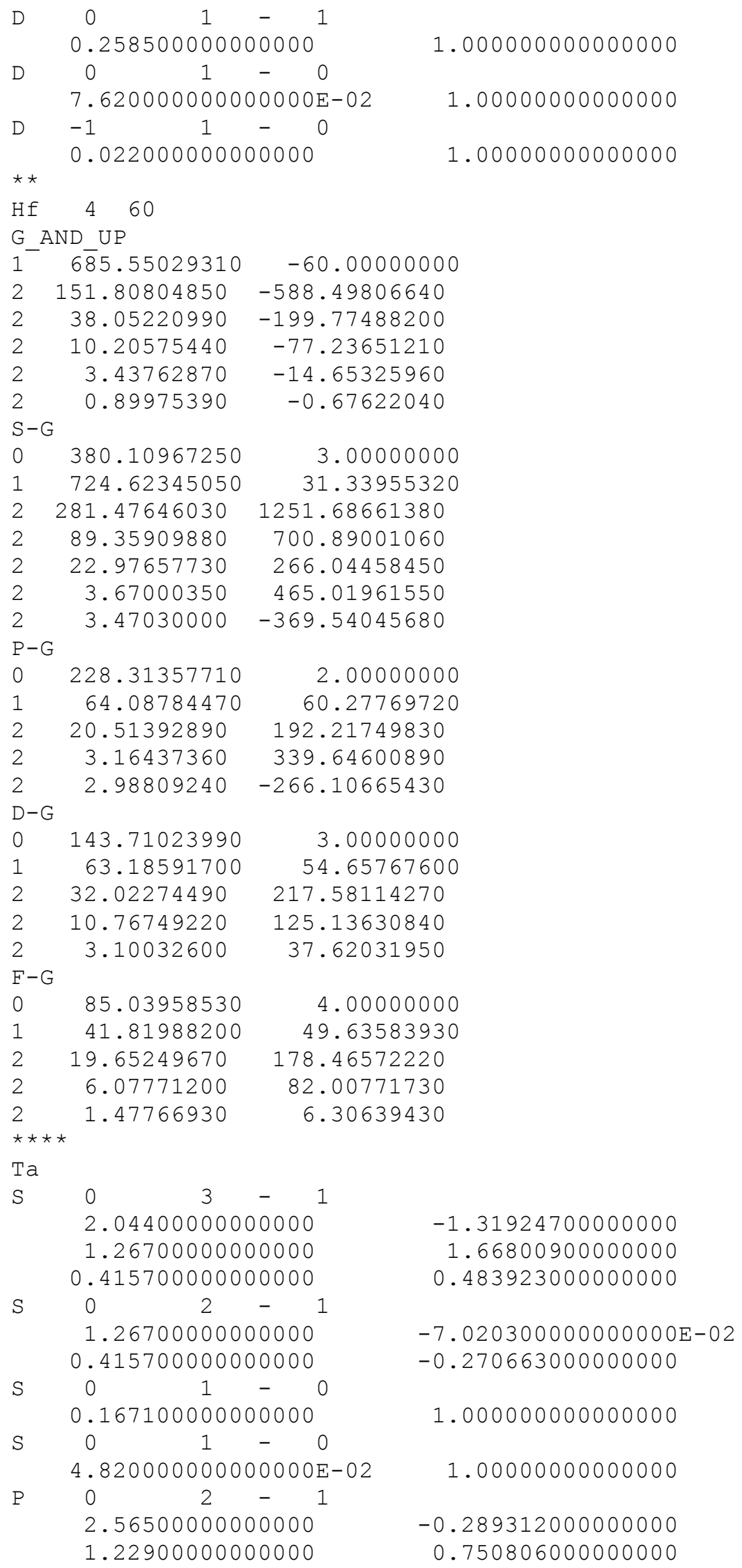




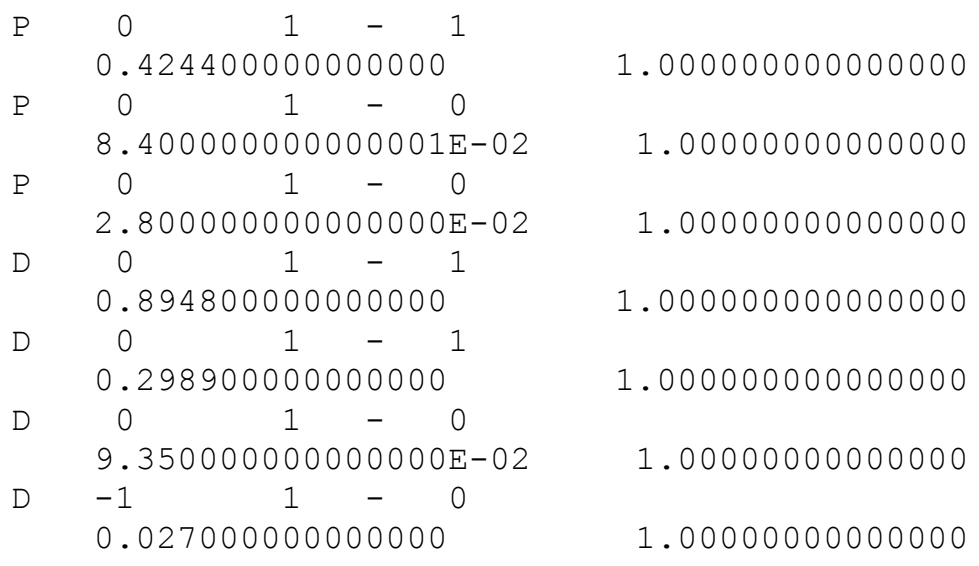

$\star \star$

$\mathrm{Ta} \quad 4 \quad 60$

G AND UP

$1^{-} \quad 7 \overline{6} 8.22675330$

173.59402180

$-60.00000000$

$43.70422680-221.17505030$

$11.61813190 \quad-83.47696830$

$4.03146180-17.83702750$

$1.09053780 \quad-0.81606090$

$S-G$

$0 \quad 365.41115590$

697.13009150

271.78103790

.00000000

87.30742630

22.74923390

3.86800820

3. 67188910

33.97989850

1242.57762440

706.16638840

276.24645630

513.22831320

$-412.96019160$

$\mathrm{P}-\mathrm{G}$

o 228.21073670

2.00000000

62.61854620

61.81741630

19.64656700

194.49754790

3.36515590

307.83466710

3.14972360

$-230.63463810$

$D-G$

$0 \quad 151.27555540$

67.97949110

3.00000000

34.74747060

55.26709780

11.56564250

236.29575450

3.29301480

134.47550720

41.14011830

$\mathrm{F}-\mathrm{G}$

$0 \quad 82.83680290$

38.07198720

4.00000000

17.45470340

50.75337600

5.67859310

162.03128770

1.58140410

72.06192980

6.00098950

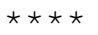

W

$\begin{array}{lllll}\mathrm{S} & 0 & 3 & -\end{array}$

2.13700000000000

$-1.40450800000000$

1.34700000000000

1.76578400000000

0.436600000000000

S

$2-1$

0.468443000000000
1.34700000000000
$-7.827099999999999 \mathrm{E}-02$ 


\begin{tabular}{|c|c|c|c|}
\hline \multicolumn{3}{|c|}{0.436600000000000} & \multirow[t]{2}{*}{-0.286869000000000} \\
\hline$S$ & $0 \quad 1$ & -0 & \\
\hline & \multicolumn{2}{|c|}{0.188300000000000} & \multirow{2}{*}{1.000000000000000} \\
\hline & $0 \quad 1$ & -0 & \\
\hline & \multicolumn{2}{|c|}{$5.180000000000000 \mathrm{E}-02$} & \multirow[t]{2}{*}{1.00000000000000} \\
\hline & $0 \quad 2$ & -1 & \\
\hline & \multicolumn{2}{|c|}{3.00500000000000} & \multirow{3}{*}{$\begin{array}{r}-0.238809000000000 \\
0.731060000000000\end{array}$} \\
\hline & \multicolumn{2}{|c|}{1.22800000000000} & \\
\hline & $0 \quad 1$ & -1 & \\
\hline & \multicolumn{2}{|c|}{0.441500000000000} & \multirow[t]{2}{*}{1.000000000000000} \\
\hline & $0 \quad 1$ & -0 & \\
\hline & \multicolumn{2}{|c|}{$9.000000000000000 \mathrm{E}-02$} & \multirow[t]{2}{*}{1.00000000000000} \\
\hline & $0 \quad 1$ & -0 & \\
\hline & \multicolumn{2}{|c|}{$2.800000000000000 \mathrm{E}-02$} & \multirow[t]{2}{*}{1.00000000000000} \\
\hline & $0 \quad 1$ & -1 & \\
\hline & \multicolumn{2}{|c|}{0.951900000000000} & \multirow[t]{2}{*}{1.000000000000000} \\
\hline & $0 \quad 1$ & $\begin{array}{llll}- & 1\end{array}$ & \\
\hline & \multicolumn{2}{|c|}{0.327000000000000} & \multirow[t]{2}{*}{1.000000000000000} \\
\hline & $\begin{array}{ll}0 & 1\end{array}$ & -0 & \\
\hline & 0.1054000000 & 00000 & 1.00000000000000 \\
\hline & $-1 \quad 1$ & -0 & \\
\hline & 0.0310000000 & 00000 & 1.00000000000000 \\
\hline & & & \\
\hline & 460 & & \\
\hline & AND_UP & & \\
\hline & 839.44891200 & -60.00 & 0000 \\
\hline & 192.85324820 & -664.198 & 200 \\
\hline & 48.66519740 & -238.614 & 510 \\
\hline & 12.92217270 & -88.4192 & 070 \\
\hline & 4.57488900 & -20.6062 & 260 \\
\hline & 1.26817960 & -0.9283 & 920 \\
\hline & & & \\
\hline 0 & 313.42675180 & 3.000 & 0000 \\
\hline & 699.31554620 & 39.119 & 9670 \\
\hline 2 & 259.89237410 & 1180.9692 & 740 \\
\hline & 85.49999800 & 728.9564 & 100 \\
\hline 2 & 22.76359250 & 293.5591 & 400 \\
\hline 2 & 4.07643170 & 562.6731 & 1930 \\
\hline 2 & 3.88271620 & -457.3807 & 850 \\
\hline & & & \\
\hline & 224.39264240 & 2.000 & 0000 \\
\hline 1 & 61.67369310 & 63.894 & 3930 \\
\hline 2 & 19.14690430 & 205.8901 & 370 \\
\hline 2 & 3.55657100 & 312.1427 & 530 \\
\hline 2 & 3.32632100 & -231.3961 & 810 \\
\hline & & & \\
\hline 0 & 161.52789580 & 3.000 & 0000 \\
\hline 1 & 75.58146070 & 55.331 & 2560 \\
\hline 2 & 38.91158520 & 267.1976 & 530 \\
\hline 2 & 12.54262710 & 146.8485 & 780 \\
\hline 2 & 3.46151870 & 44.1055 & 430 \\
\hline & & & \\
\hline 0 & 91.21027270 & 4.000 & 0000 \\
\hline 1 & 45.41527560 & 50.306 & 5230 \\
\hline 4 & 22.04529670 & 190.7363 & 980 \\
\hline 2 & 6.98104130 & 91.7605 & 520 \\
\hline & 1.83804800 & 8.4247 & 320 \\
\hline
\end{tabular}




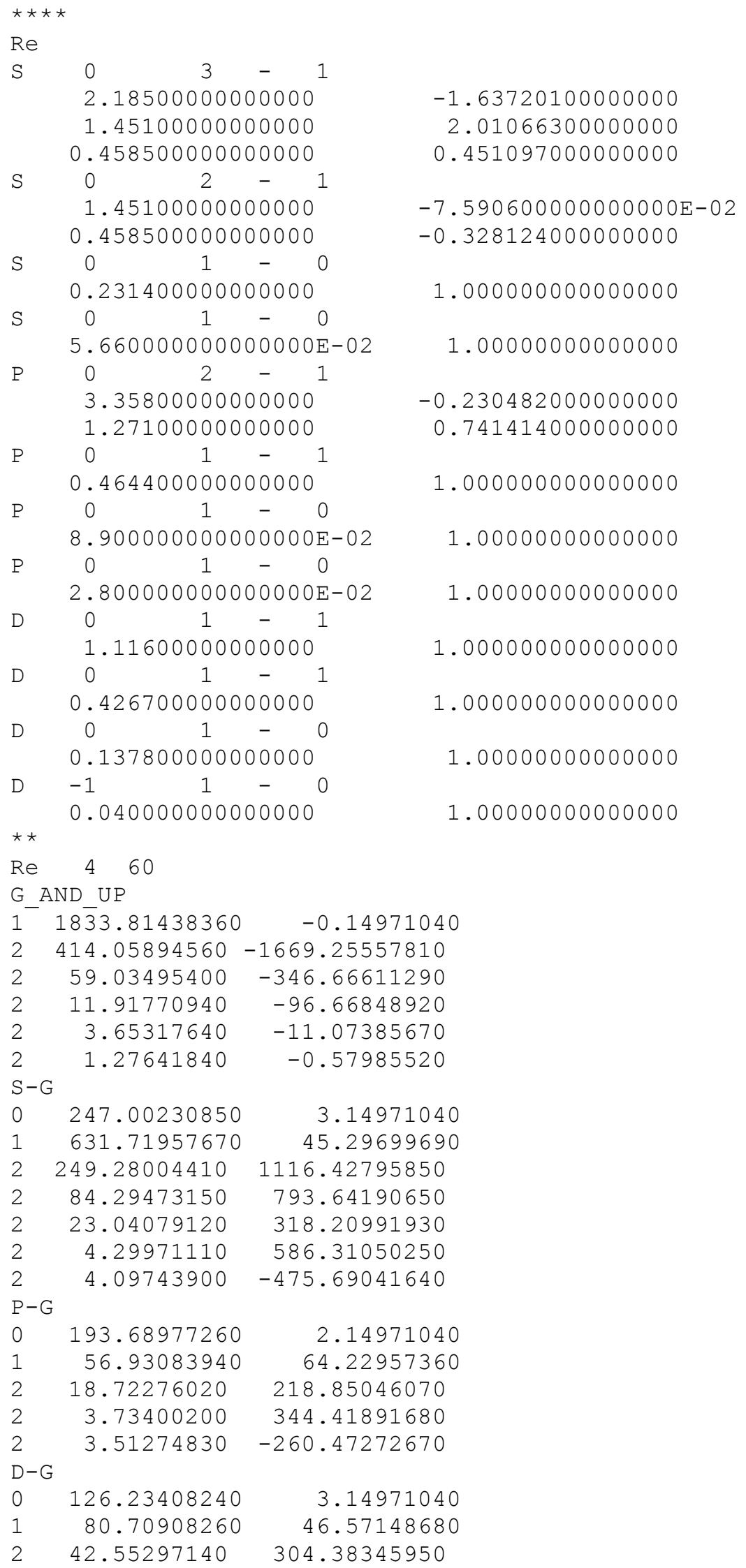




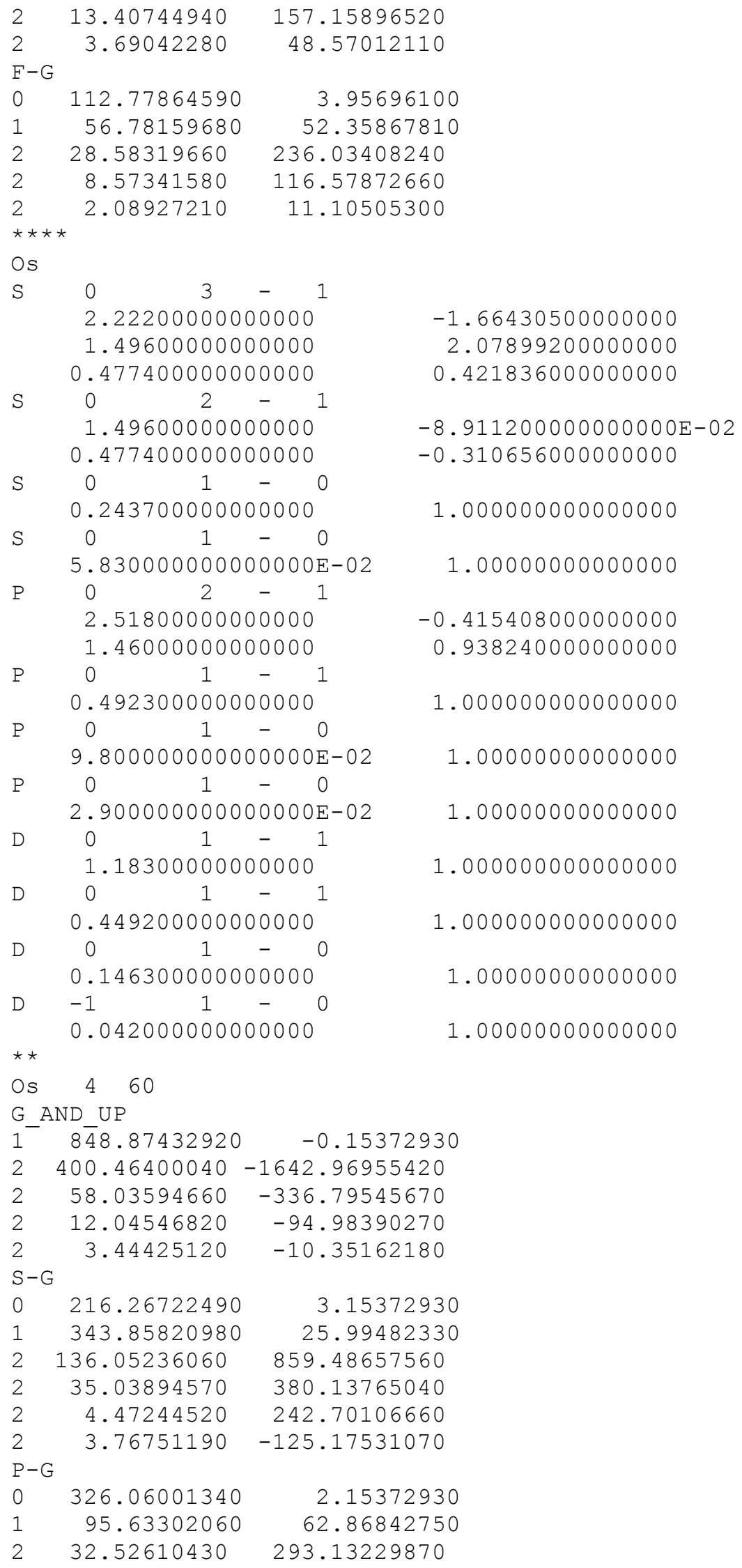




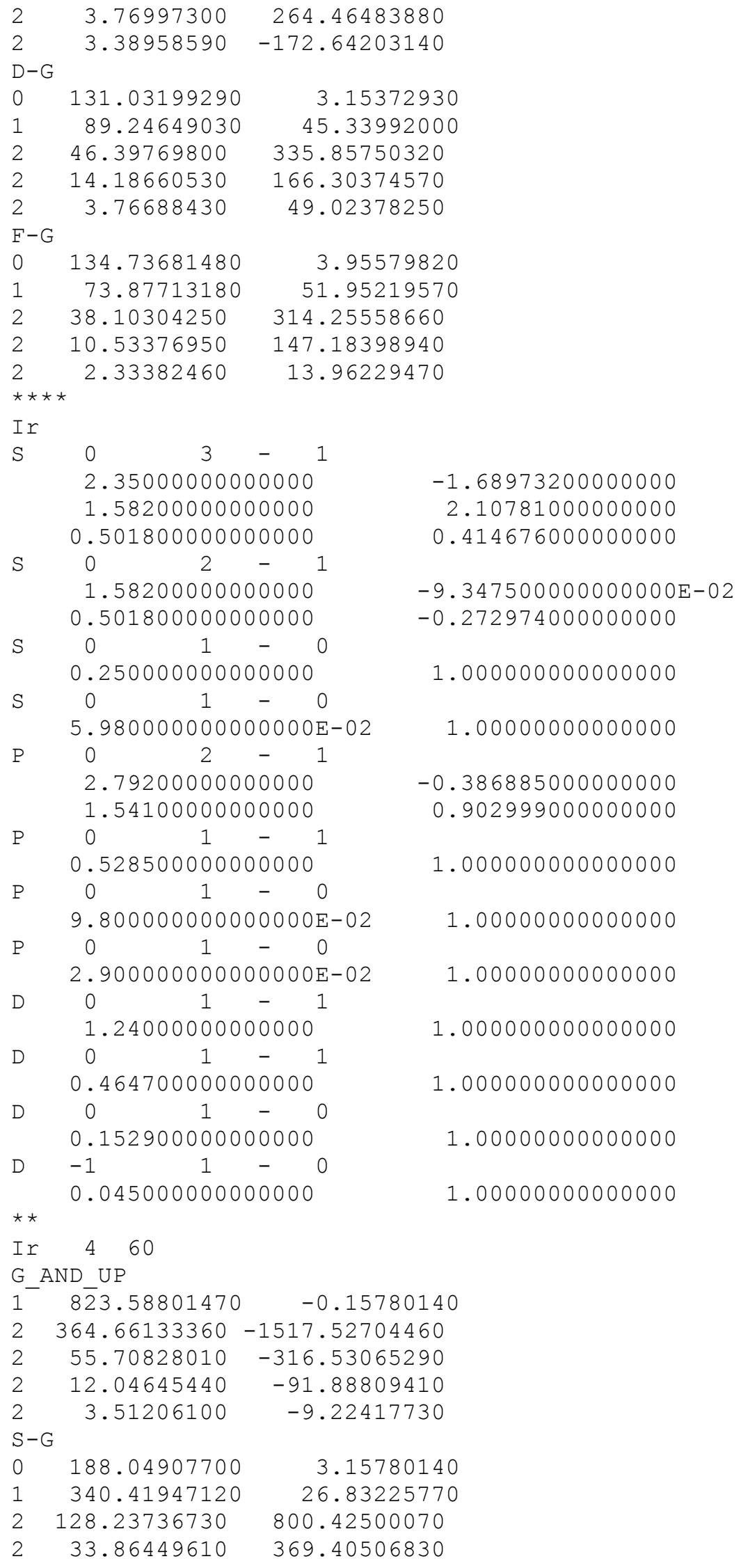




$\begin{array}{lrr}2 & 4.75600050 & 242.41718990 \\ 2 & 3.96499740 & -118.21732820 \\ \mathrm{P}-\mathrm{G} & & \\ 0 & 289.72911390 & 2.15780140 \\ 1 & 87.46337890 & 61.96786100 \\ 2 & 30.43637660 & 269.05819860 \\ 2 & 4.05534120 & 231.16547930 \\ 2 & 3.55253410 & -133.69526670 \\ \mathrm{D}-\mathrm{G} & & \\ 0 & 136.40171060 & 3.15780140 \\ 1 & 95.07769250 & 45.93498030 \\ 2 & 49.22584100 & 359.03446680 \\ 2 & 15.08741450 & 176.47401190 \\ 2 & 4.04057640 & 54.51552860 \\ \mathrm{~F}-\mathrm{G} & & \\ 0 & 127.35079080 & 3.95461970 \\ 1 & 66.23643740 & 52.97736550 \\ 2 & 34.42992290 & 274.86433830 \\ 2 & 10.19957210 & 137.20473380 \\ 2 & 2.54097020 & 14.86333050 \\ \star \star \star * & & \end{array}$

$\mathrm{Pt}$

S $\quad 0 \quad 3 \quad-1$

2.54700000000000

$-1.48483800000000$

1.61400000000000

1.92412600000000

0.516700000000000

0.390069000000000

1.61400000000000

0.516700000000000

$0 \quad 1 \quad-\quad 0$

$-9.382600000000001 \mathrm{E}-02$

$-0.295580000000000$

$0.265100000000000 \quad 1.000000000000000$

$0-1-0$
$5.800000000000000 \mathrm{E}-02$
1.00000000000000

P

$0 \quad 2 \quad-\quad 1$

2.91100000000000

$-0.521248000000000$

1.83600000000000

$\begin{array}{lllll}\mathrm{P} & 0 & 1 & - & 1\end{array}$

0.960745000000000

0.598200000000000

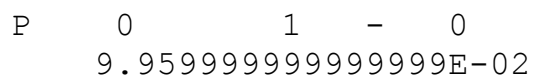

1.000000000000000

$\mathrm{P}$

2. $900000000000000 E-02$

1.00000000000000

1.00000000000000

1.24300000000000

1.000000000000000

D $\quad 0 \quad 1 \quad-1$

0.427100000000000

D $\quad 0 \quad 1 \quad-0$

0.137000000000000

$\begin{array}{lllll}\mathrm{D} & -1 & 1 & - & 0\end{array}$

0.038000000000000

1.000000000000000

1.00000000000000

1.00000000000000

$\star \star$

Pt 460

$G$ AND UP

$1^{-} 7 \overline{2} 8.93940560 \quad-0.16192680$

$2 \quad 320.65678000-1320.28738520$

$2 \quad 52.86801740-298.31781350$

$2 \quad 12.02801280 \quad-87.58370650$ 


\begin{tabular}{lrr}
2 & 3.52389130 & \multicolumn{1}{c}{-8.14932740} \\
$\mathrm{~S}-\mathrm{G}$ & & \\
0 & 409.44373580 & 2.73342180 \\
1 & 274.54192310 & 59.70243290 \\
2 & 127.56585700 & 891.45895500 \\
2 & 32.90366310 & 368.44676560 \\
2 & 5.05938800 & 238.02630900 \\
2 & 4.15065560 & -107.05564540 \\
$\mathrm{P}-\mathrm{G}$ & & \\
0 & 466.17288920 & 1.88785680 \\
1 & 120.78882590 & 76.01386290 \\
2 & 36.41187910 & 343.55111160 \\
2 & 5.69854080 & 119.49117860 \\
$\mathrm{D}-\mathrm{G}$ & & \\
0 & 249.56507630 & 2.93436780 \\
1 & 126.66785850 & 59.33065710 \\
2 & 63.14305860 & 452.44451940 \\
2 & 17.90594700 & 210.47694790 \\
2 & 4.22393730 & 58.62541120 \\
$\mathrm{~F}-\mathrm{G}$ & & \\
0 & 121.81587990 & 3.95342530 \\
1 & 60.87570300 & 53.85551820 \\
2 & 31.47671470 & 247.43051330 \\
2 & 9.88117510 & 127.81879760 \\
2 & 2.73198740 & 15.37720460 \\
$\star \star \star \star$ & &
\end{tabular}

$\mathrm{Au}$

$\begin{array}{llcc}0 & 3 & - & 1\end{array}$

2.80900000000000
1.59500000000000

$-1.20303700000000$

0.532700000000000

1.67516200000000

1.59500000000000

0.352291000000000

0.532700000000000

$\begin{array}{lll}0 & - & 0\end{array}$

0.282600000000000

$\begin{array}{llll}0 & 1 & - & 0\end{array}$
$5.980000000000000 E-02$
1.00000000000000

$-0.100923000000000$

$-0.283260000000000$

1.000000000000000

P

3. 68400000000000

$-0.278885000000000$

1.66600000000000

$\mathrm{P} \quad 0 \quad 1 \quad-1$

0.598900000000000

0.777981000000000

$9.770000000000000 \mathrm{E}-0$

1.000000000000000

1.00000000000000

$\begin{array}{llll}0 & - & 0\end{array}$

1.00000000000000

$2.790000000000000 E-02$

$\begin{array}{lccc}0 & 1 & - & 1\end{array}$

1.000000000000000

$\begin{array}{lllll}\mathrm{D} & 0 & 1 & - & 1\end{array}$

0.433500000000000

1.000000000000000

D $\quad 0 \quad 1 \quad-0$

0.139600000000000

$\begin{array}{lllll}\mathrm{D} & -1 & 1 & - & 0\end{array}$

0.050000000000000

1.00000000000000

1.00000000000000

Au 460 


\begin{tabular}{|c|c|c|}
\hline \multicolumn{3}{|c|}{ G_AND_UP } \\
\hline & $6 \overline{2} 2.62879560$ & -60.00000000 \\
\hline & 136.28436070 & -555.52923120 \\
\hline & 33.15497810 & -168.00197850 \\
\hline & 9.98948950 & -63.03998750 \\
\hline & 3.04813120 & -4.25166810 \\
\hline \multicolumn{3}{|c|}{$-G$} \\
\hline & 194.73743040 & 3.00000000 \\
\hline & 351.53274470 & 38.60208800 \\
\hline & 122.32704020 & 864.83707270 \\
\hline & 32.09146170 & 374.99355200 \\
\hline & 5.24518120 & 289.79101000 \\
\hline & 4.49162230 & -152.45327730 \\
\hline \multicolumn{3}{|c|}{$P-G$} \\
\hline & 420.61588010 & 2.00000000 \\
\hline & 109.44178150 & 73.88856250 \\
\hline & 34.17142800 & 326.67298720 \\
\hline & 5.98797500 & 126.58145910 \\
\hline \multicolumn{3}{|c|}{$D-G$} \\
\hline & 219.26661580 & 3.00000000 \\
\hline & 122.72977860 & 55.67931490 \\
\hline & 63.10633690 & 449.19873350 \\
\hline & 18.36845200 & 215.02690910 \\
\hline & 4.49728440 & 64.08409950 \\
\hline \multicolumn{3}{|c|}{$F-G$} \\
\hline & 108.55060370 & 4.00000000 \\
\hline & 56.47955270 & 51.80653350 \\
\hline & 29.20691590 & 231.21831130 \\
\hline & 9.54405430 & 119.00473860 \\
\hline & 2.89651180 & 15.34241880 \\
\hline
\end{tabular}

